\title{
Ortho/Ipso Alkylborylation of Aryl Iodides
}

Xianwei Sui, Thiago A. Grigolo, Colin J. O'Connor, and Joel M. Smith*

Department of Chemistry \& Biochemistry, Florida State University,

95 Chieftan Way 118 DLC, Tallahassee, Florida 32306, United States

\section{SUPPORTING INFORMATION}




\section{Table of Contents}

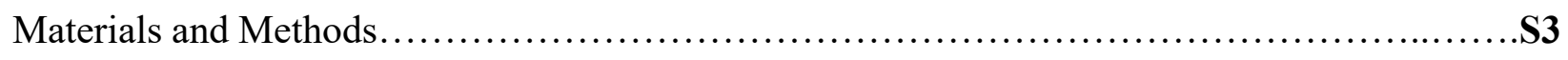

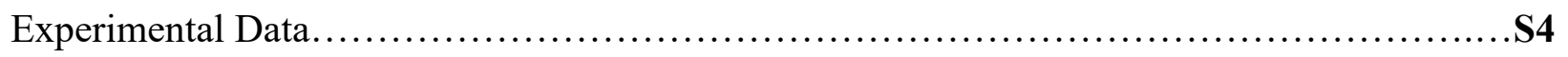

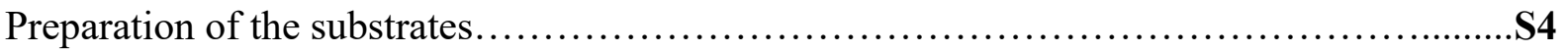

Optimization details and notes of the reaction conditions............................S6

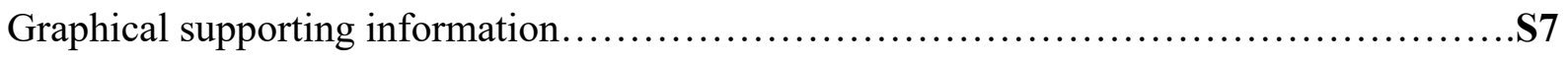

General Procedure for Alkylborylation............................................S8

Arene scope of Alkylborylation ....................................................

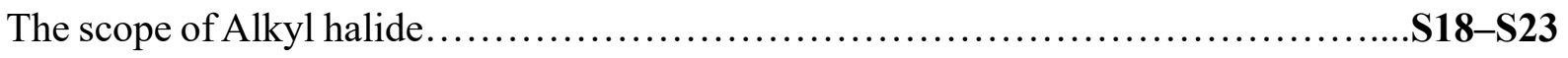

Diversification of the Alkyaborylation products................................. S23 26

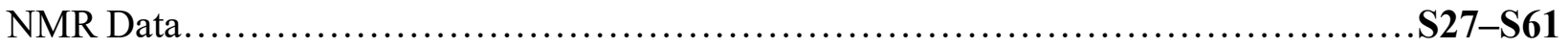

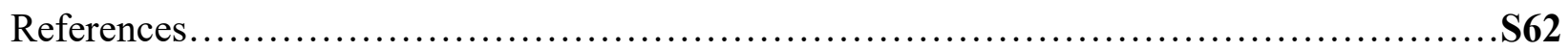




\section{Materials and Methods}

Nuclear magnetic resonance spectra were recorded on Bruker 400, 500, 600 and $700 \mathrm{MHz}$ instruments internally referenced to tetramethylsilane $(0.0 \mathrm{ppm})$ or residue of $\mathrm{CDCl}_{3}(7.26 \mathrm{ppm}$ for $1 \mathrm{H}$, and $77.00 \mathrm{ppm}$ for $13 \mathrm{C}$ ) signal. The following abbreviations were used to explain multiplicities: $\mathrm{s}=$ singlet, $\mathrm{d}=$ doublet, $\mathrm{t}=$ triplet, $\mathrm{q}=$ quartet, $\mathrm{m}=$ multiplet, $\mathrm{br}=$ broad, $\mathrm{c}=$ complex. High-resolution mass spectra (HRMS) were recorded on direct analysis in real time (DART) ionization techniques (Agilent). All reagents were purchased at the highest commercial quality and used without further purification unless otherwise stated. Paladium(II) acetate, methyl chloroacetate, ethyl chloroacetate, benzyl chloride, 2-iodotoluene, 2-bromotoluene, 2iodoisopropylbenzene, 2-iodotrifluorobenzene, 1-iodonaphthalene, 2-iodoanisole, 2-iodobiphenyl, 1-chloro-2-iodobenzene, 2-chloro-6-iodotoluene, 4-iodoanisole, copper(II) acetate, sodium azide, N-bromosuccinimide, copper(II) chloride, potassium carbonate, cesium carbonate and bis(pinacolato)diboron were purchased from Oakwood Chemical. Di-tert-butyl dicarbonate, benzyl alcohol, morpholine, copper(II) bromide, hydroxylamine hydrochloride, furan, sodium hydroxide $(60 \%$ dispersion in mineral oil), methyl iodide, potassium hydrogen phosphate, triphenyl phosphine, Devephos, Ruphos, $\mathrm{P}(\text { furyl })_{3}, \mathrm{P}(\mathrm{p}-\mathrm{MeOPhenyl})_{3}$, S-phos and norbornene were purchased from Sigma-Aldrich. Benzyl amine, n-butyllithium (2.5 M in hexanes), acetic acid and tripotassium phosphate were purchased from Across Organics. Cyclohexanol, sodium thiosulfate and sodium acetate were purchased from J.T. Baker. Cyclohexanone and potassium bicarbonate were purchased from Mallinckodt Chemicals. Chloroacetyl chloride was purchased from Beantown Chemical. All solvents were dried by passage through activated alumina using a solvent system purchased from Pure Process Technology Inc. All reactions were performed under an inert atmosphere of dry nitrogen in flame-dried glassware, unless otherwise stated. Room temperature reactions were performed between $22-25^{\circ} \mathrm{C}$. TLC was performed using $0.25 \mathrm{~mm}$ E. Merck silica plates (60F-254), using short-wave UV light as the visualizing agent, and phosphomolybdic acid, $p$-anisaldehyde, or $\mathrm{KMnO}_{4}$ and heat as developing agents. 


\section{Experimental Data}

\section{Preparation of substrates}<smiles>O=C(CCl)OCCCCC(=O)c1ccccc1</smiles>

ref. 1<smiles>O=C(CCl)O[GeH3]</smiles>

ref. 1<smiles>O=C(CCl)N1CCOCC1</smiles>

ref. 2<smiles>O=C(CCl)NCc1ccccc1</smiles>

ref. 2<smiles>Cc1cc(NC(=O)OC(C)(C)C)ccc1I</smiles>

ref. 3<smiles>COc1cccc(I)c1OC</smiles>

ref. 4<smiles>COC(=O)c1ccc(C)c(I)c1</smiles>

ref. 5<smiles>O=C(OCc1ccccc1)c1ccc(I)cc1</smiles>

ref.6

Figure S1. Syntheses of known substrates used in this study<smiles>NNc1ccccc1I</smiles>
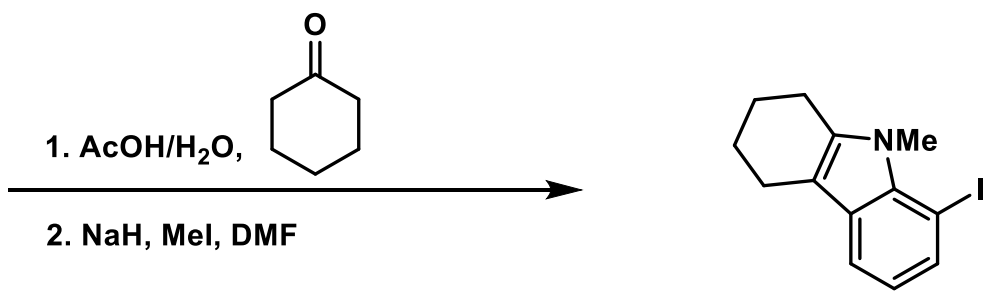

The preparation of 1,2,3,4-tetrahydro-8-iodo-9-methylcarbazole followed amodified literature procedure $^{7}$. To a solution of hydrazine (500 mg, $2.14 \mathrm{mmol}, 1$ equiv.) in $\mathrm{AcOH} / \mathrm{H}_{2} \mathrm{O}(8 \mathrm{~mL} / 8 \mathrm{~mL})$ was added cyclohexanone (209 mg, $2.14 \mathrm{mmol}, 1$ equiv.), and the reaction was refluxed at $100{ }^{\circ} \mathrm{C}$ for $7 \mathrm{~h}$. The mixture was washed with sat. $\mathrm{NaHCO}_{3}(50 \mathrm{ml})$ extracting with ethyl acetate $(3 \times 30$ $\mathrm{mL})$. The organic layer was washed with brine $(30 \mathrm{~mL})$, dried over $\mathrm{Na}_{2} \mathrm{SO}_{4}$, concentrated, and the residue was filtered by a short silica gel column, concentrated, and used for next step without further purification. To the solution of reside in DMF $(10 \mathrm{~mL})$ was added $\mathrm{NaH}(61.6 \mathrm{mg}, 2.56$ mmol, 1.2 equiv.) at $0{ }^{\circ} \mathrm{C}$ and the mixture was stirred at $0{ }^{\circ} \mathrm{C}$ for another $20 \mathrm{mins}$. MeI (447 mg, $3.21 \mathrm{mmol}, 1.5$ equiv.) was added and the reaction was stirred at room temperature for $3 \mathrm{~h}$ before pouring in to $40 \mathrm{ml}$ water. The mixture was extracted with ethyl acetate $(3 \times 30 \mathrm{~mL})$. The organic layer was washed with brine $(30 \mathrm{~mL})$, dried over $\mathrm{Na}_{2} \mathrm{SO}_{4}$, concentrated, and the residue was 
purified by column chromatography (silica gel, $5 \%$ ethyl acetate in hexanes as eluent) to give the product ( $452 \mathrm{mg}, 68 \%$ for two steps) $\left(\mathrm{R}_{\mathrm{f}}=0.45,5 \%\right.$ ethyl acetate/hexanes). 1 H NMR (400 MHz, CDCl3) $\delta 7.58(\mathrm{dd}, J=1.2,7.6 \mathrm{~Hz}, 1 \mathrm{H}), 7.40(\mathrm{dd}, J=1.2,7.6 \mathrm{~Hz}, 1 \mathrm{H}), 6.74(\mathrm{t}, J=7.6 \mathrm{~Hz}, 1 \mathrm{H})$, 4.00 (s, 3 H), 2.74-2.59 (m, 4 H), 2.00-1.89 (m, 2 H), 1.89-1.78 (m, 2 H); 13C NMR (150 MHz, CDC13) $\delta 137.7,135.7,133.2,129.9,120.4,117.7,109.6,72.6,31.7,23.3,23.1,22.6,20.7$;

HRMS-DART (m/z): calcd for $\mathrm{C}_{13} \mathrm{H}_{14} \mathrm{IN}[\mathrm{M}+\mathrm{H}]^{+} 312.0255$, found 312.0259 . 


\section{Optimizaiton details and notes of the reaction conditions}

Table S1. Optimization details of the reaction conditions ${ }^{\mathrm{a}}$

\begin{tabular}{ccccc}
\hline & & & \\
additive, base, solvent \\
$\mathbf{9 5}{ }^{\circ} \mathbf{C}, \mathbf{1 6}$ h
\end{tabular}

Notes:

1. The use of dried KOAc (moisture sensitive) is necessary for the reaction reproducibility.

2. Too much or less norbornene (as well as solvent) is not good for reaction reproducibility. 


\section{Graphical supporting information}
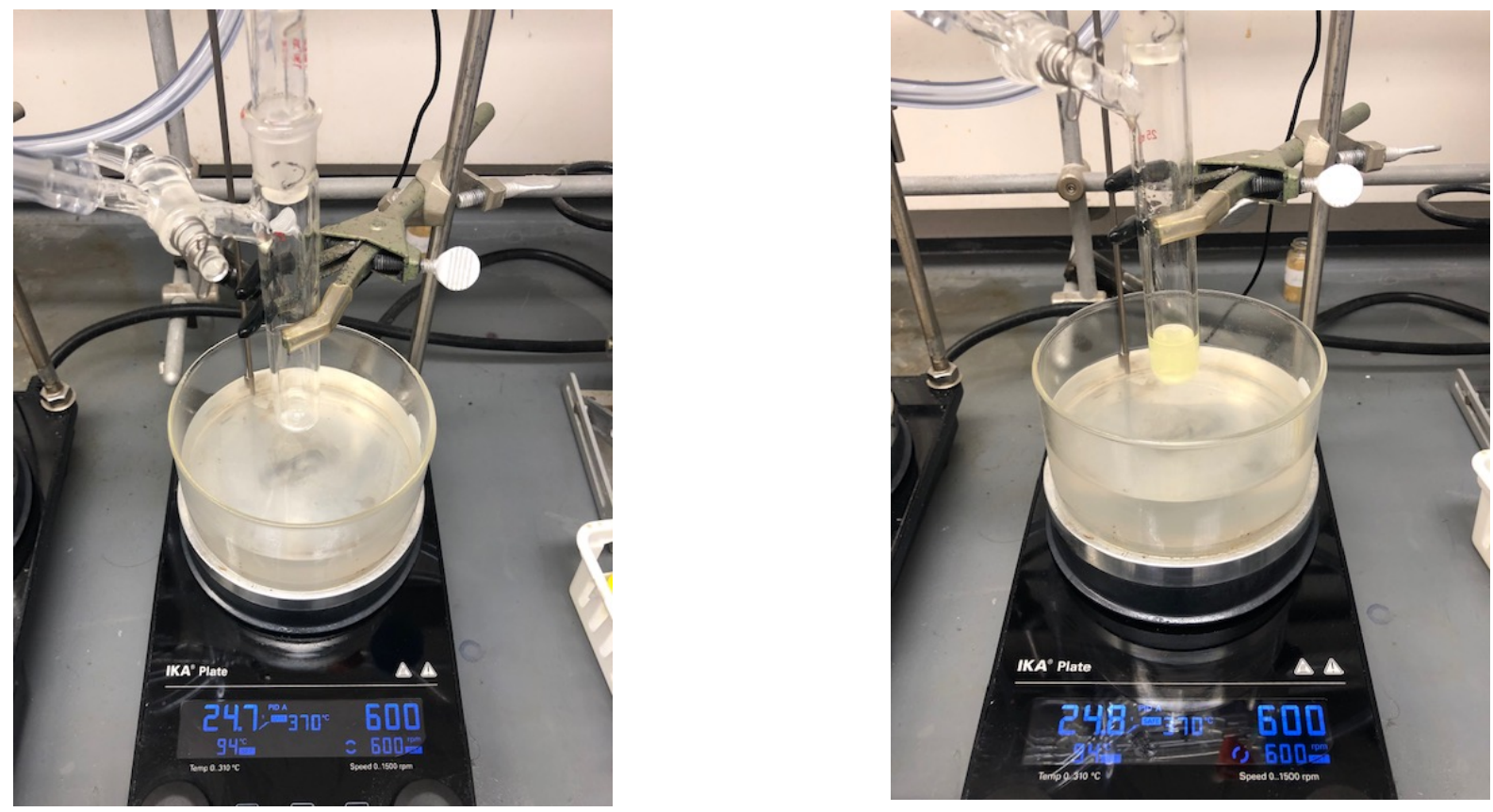

(a) A flamed-dried Schlenk tube was placed under Ar.

(b) All the reagents added to the tube.

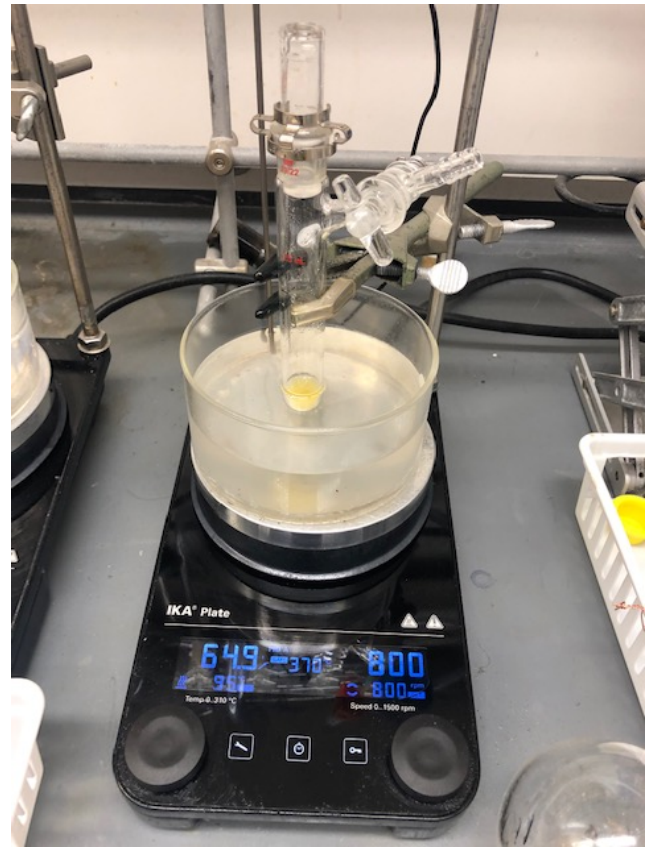

(c) After heating mixture at $95^{\circ} \mathrm{C}$.

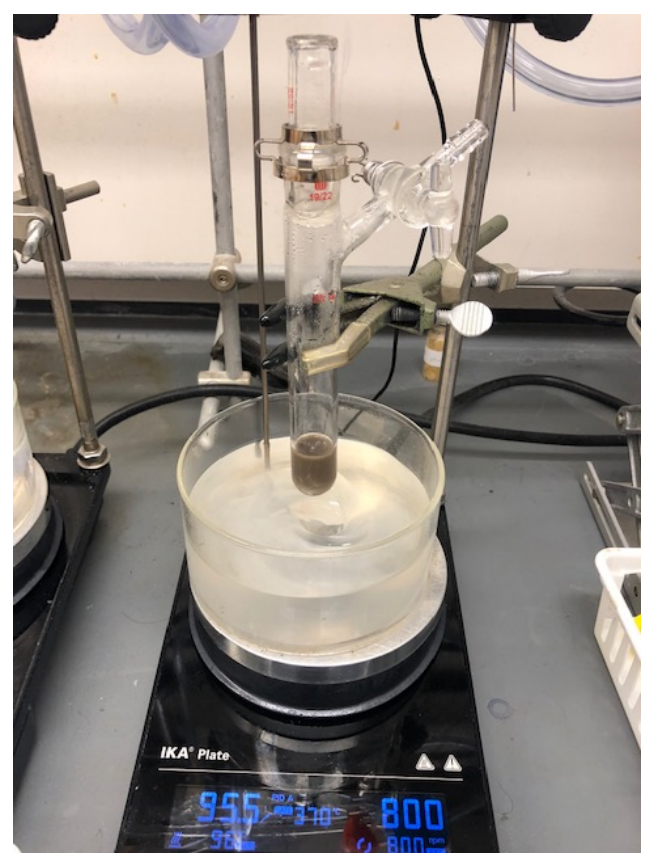

(d) The reaction complete after $15 \mathrm{~h}$. 


\section{General Procedure for Alkylborylation of aryl iodides}

$\mathrm{Pd}(\mathrm{OAc})_{2}(2.5 \mathrm{mg}, 0.0112 \mathrm{mmol}, 7 \%)$ and $\mathrm{PPh}_{3}(5.9 \mathrm{mg}, 0.0224 \mathrm{mmol}, 14 \%)$ were added to a Schlenk tube followed by $\mathrm{MeCN}(5.0 \mathrm{ml})$, and the mixture was stirred for $10 \mathrm{mins}$ at room temperature. Aryl halide (0.160 mmol, 1.0 equiv.), norbornene (22.6 mg, $0.240 \mathrm{mmol}, 1.5$ equiv.), $\mathrm{K}_{2} \mathrm{CO}_{3}$ (66.2 mg, $0.480 \mathrm{mmol}, 3.0$ equiv.) and KOAc (102 mg, $1.04 \mathrm{mmol}, 6.5$ equiv.) were added and the mixture was stirred for another $10 \mathrm{mins}$ at room temperature. The alkyl halide $(0.640 \mathrm{mmol}$, 4.0 equiv.) was then added and the mixture was stirred for 10 mins at $40{ }^{\circ} \mathrm{C}$ before bis(pinacolato)diboron ( $81.3 \mathrm{mg}, 0.320 \mathrm{mmol}, 2.0$ equiv.) was added, and reaction was stirred for $16 \mathrm{~h}$ at $95^{\circ} \mathrm{C}$. The mixture was poured into water $(20 \mathrm{~mL})$ and extracted with ethyl acetate $(3 \times 15$ $\mathrm{ml})$. The organic layer was washed with brine $(15 \mathrm{~mL})$, dried over $\mathrm{Na}_{2} \mathrm{SO}_{4}$, filtered and concentrated. The reside was then purified by column chromatography on silica gel to give the alkylborylation product.

\section{Arene scope of Alkylborylation}

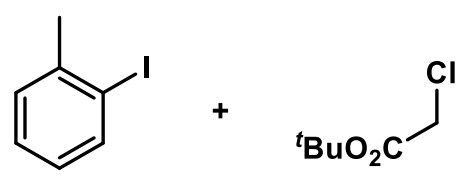

11

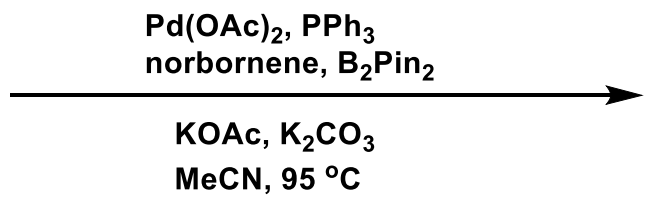

$\mathrm{MeCN}, 95^{\circ} \mathrm{C}$

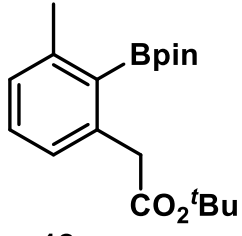

12

Compound 12 (using 2-iodotoluene) was synthesized following the General Procedure for the Alkylborylation of aryl iodidess. The reaction of 2-iodotoluene (34.9 $\mathrm{mg}, 0.160 \mathrm{mmol}, 1.0$ equiv.), 11 (96.0 mg, $0.640 \mathrm{mmol}, 4.0$ equiv.), $\mathrm{Pd}(\mathrm{OAc})_{2}$ (2.5 mg, $0.0112 \mathrm{mmol}, 7$ \%), $\mathrm{PPh}_{3}(5.9$ mg, $0.0224 \mathrm{mmol}, 14 \%$ ), norbornene (22.6 mg, $0.240 \mathrm{mmol}, 1.5$ equiv.), $\mathrm{K}_{2} \mathrm{CO}_{3}$ (66.2 mg, 0.480 mmol, 3.0 equiv.), KOAc (102 mg, 1.04 mmol, 6.5 equiv.) and $B_{2} \operatorname{pin}_{2}$ (81.3 mg, 0.320 mmol, 2.0 equiv.) in $\mathrm{MeCN}(5.0 \mathrm{~mL})$ delivered 12 (38.2 mg, 72\%) after purification via silica gel column chromatography with $2.5 \%$ ethyl acetate/hexanes $\left(\mathrm{R}_{\mathrm{f}}=0.4,5 \%\right.$ ethyl acetate/hexanes $) . \mathbf{1 H}$ NMR $\left(400 \mathrm{MHz}, \mathrm{CDCl}_{3}\right) \delta 7.20(\mathrm{t}, J=7.6 \mathrm{~Hz}, 1 \mathrm{H}), 7.03(\mathrm{dd}, J=2.4,7.2 \mathrm{~Hz}, 2 \mathrm{H}), 3.69$ (s, $\left.2 \mathrm{H}\right), 2.46$ (s, $3 \mathrm{H}), 1.43$ (s, $9 \mathrm{H}), 1.38$ (s, $12 \mathrm{H}) ; 13 \mathrm{C} \mathrm{NMR}\left(100 \mathrm{MHz}, \mathrm{CDCl}_{3}\right) \delta$ 171.4, 142.9, 139.2, 129.4, 128.1, 127.1, 83.6, 80.3, 42.5, 28.1, 25.0, 22.5; HRMS-DART (m/z): calculated for $\mathrm{C}_{13} \mathrm{H}_{18} \mathrm{O}_{2} \mathrm{~B}$ 
$[\mathrm{M}+\mathrm{H}]^{+}$333.2232, found 333.2238.

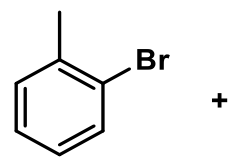

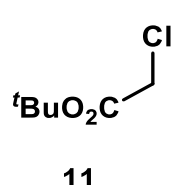

11

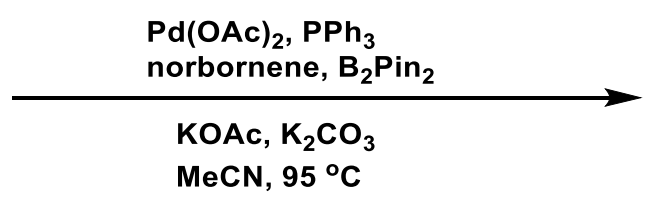

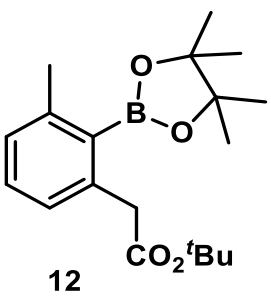

Compound 12 (using 2-bromotoluene) was synthesized following the General Procedure for the Alkylborylation of aryl iodidess. The reaction of 2-bromotoluene (27.2 $\mathrm{mg}, 0.160$ mmol, 1.0 equiv.), 11 (96.0 mg, $0.640 \mathrm{mmol}, 4.0$ equiv.), $\mathrm{Pd}(\mathrm{OAc})_{2}(2.5 \mathrm{mg}, 0.0112 \mathrm{mmol}, 7 \%$ ), $\mathrm{PPh}_{3}$ (5.9 mg, $0.0224 \mathrm{mmol}, 14 \%$ ), norbornene (22.6 mg, $0.240 \mathrm{mmol}, 1.5$ equiv.), $\mathrm{K}_{2} \mathrm{CO}_{3}(66.2$ $\mathrm{mg}, 0.480 \mathrm{mmol}, 3.0$ equiv.), KOAc (102 mg, $1.04 \mathrm{mmol}, 6.5$ equiv.) and $\mathrm{B}_{2} \mathrm{pin}_{2}(81.3 \mathrm{mg}, 0.320$ mmol, 2.0 equiv.) in $\mathrm{MeCN}(5.0 \mathrm{~mL})$ delivered $12(11.7 \mathrm{mg}, 22 \%)$ after purification via silica gel column chromatography with $2.5 \%$ ethyl acetate/hexanes.

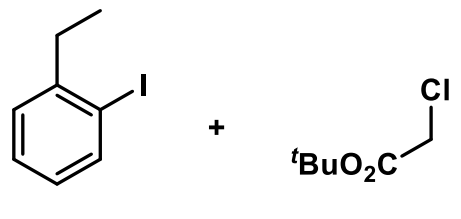

11

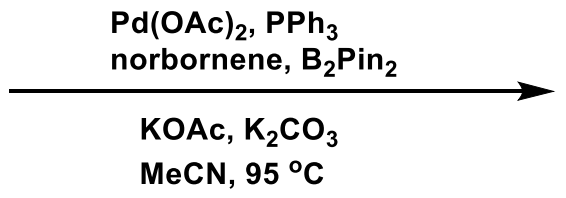

$\operatorname{MeCN}, 95^{\circ} \mathrm{C}$

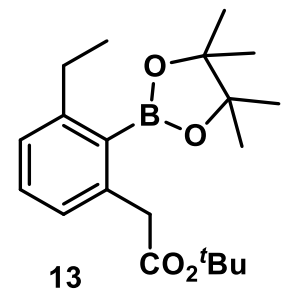

13

Compound 13 was synthesized following the General Procedure for the Alkylborylation

of aryl iodidess. The reaction of 1-ethyl-2-iodobenzene ( $37.1 \mathrm{mg}, 0.160 \mathrm{mmol}, 1.0$ equiv.), 11 (96.0 mg, 0.640 mmol, 4.0 equiv.), $\mathrm{Pd}(\mathrm{OAc})_{2}$ (2.5 mg, $0.0112 \mathrm{mmol}, 7$ \%), $\mathrm{PPh}_{3}$ (5.9 mg, 0.0224 mmol, $14 \%$ ), norbornene (22.6 mg, $0.240 \mathrm{mmol}, 1.5$ equiv.), $\mathrm{K}_{2} \mathrm{CO}_{3}(66.2 \mathrm{mg}, 0.480 \mathrm{mmol}, 3.0$ equiv.), KOAc (102 mg, $1.04 \mathrm{mmol}, 6.5$ equiv.) and $\mathrm{B}_{2} \operatorname{pin}_{2}$ (81.3 mg, $0.320 \mathrm{mmol}, 2.0$ equiv.) in MeCN (5.0 mL) delivered $13(41.5 \mathrm{mg}, 75 \%)$ after purification via silica gel column chromatography with $2.5 \%$ ethyl acetate/hexanes ( $R_{\mathrm{f}}=0.4,5 \%$ ethyl acetate/hexanes). 1 H NMR $\left(400 \mathrm{MHz}, \mathrm{CDCl}_{3}\right) \delta 7.24(\mathrm{t}, J=7.6 \mathrm{~Hz}, 1 \mathrm{H}), 7.05$ (t, $\left.J=7.6 \mathrm{~Hz}, 2 \mathrm{H}\right), 3.68(\mathrm{~s}, 2 \mathrm{H}), 2.77$ (q, $J=$ $7.6 \mathrm{~Hz}, 2 \mathrm{H}), 1.42(\mathrm{~s}, 9 \mathrm{H}), 1.38$ (s, $12 \mathrm{H}), 1.20$ (t, $J=7.6 \mathrm{~Hz} 3 \mathrm{H}) ; 13 \mathrm{C}$ NMR (125 MHz, CDCl$)$ $\delta 171.4,149.4,139.0,129.5,127.2,126.6,83.7,80.4,42.6,29.4,28.1,25.0,17.1$; HRMS-DART 
(m/z): calculated for $\mathrm{C}_{20} \mathrm{H}_{32} \mathrm{O}_{4} \mathrm{~B}[\mathrm{M}+\mathrm{H}]^{+} 347.2388$, found 347.2384 .
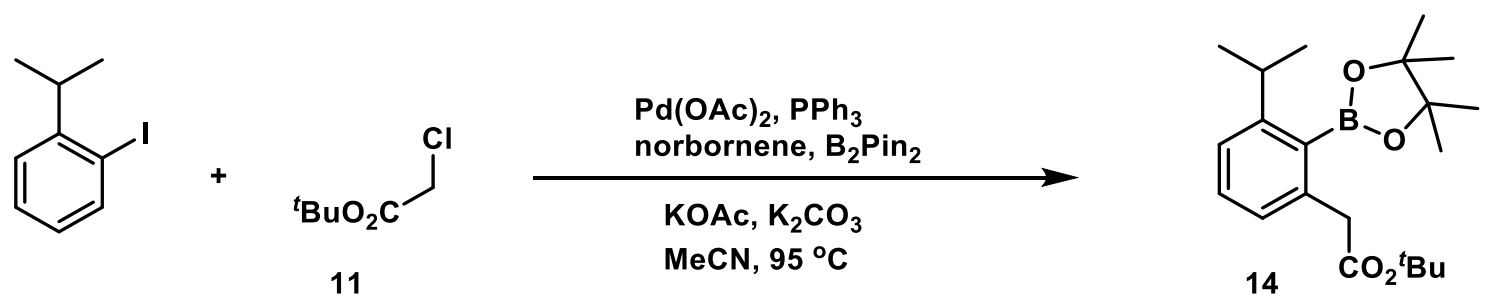

Compound 14 was synthesized following the General Procedure for the Alkylborylation of aryl iodidess. The reaction of 2-isopropyliodobenzene (39.4 mg, $0.160 \mathrm{mmol}, 1.0$ equiv.), 11 (96.0 mg, 0.640 mmol, 4.0 equiv.), $\mathrm{Pd}(\mathrm{OAc})_{2}$ (2.5 mg, $0.0112 \mathrm{mmol}, 7$ \%), $\mathrm{PPh}_{3}$ (5.9 mg, 0.0224 mmol, $14 \%$ ), norbornene (22.6 mg, $0.240 \mathrm{mmol}, 1.5$ equiv.), $\mathrm{K}_{2} \mathrm{CO}_{3}(66.2 \mathrm{mg}, 0.480 \mathrm{mmol}, 3.0$ equiv.), KOAc (102 mg, $1.04 \mathrm{mmol}, 6.5$ equiv.) and $\mathrm{B}_{2} \operatorname{pin}_{2}(81.3 \mathrm{mg}, 0.320 \mathrm{mmol}, 2.0$ equiv.) in $\mathrm{MeCN}(5.0 \mathrm{~mL})$ delivered $14(42.1 \mathrm{mg}, 73 \%)$ after purification via silica gel column chromatography with $2.5 \%$ ethyl acetate/hexanes $\left(R_{\mathrm{f}}=0.4,5 \%\right.$ ethyl acetate/hexanes $) .1 \mathbf{H}$ NMR $\left(400 \mathrm{MHz}, \mathrm{CDCl}_{3}\right) \delta 7.27(\mathrm{t}, J=8.0 \mathrm{~Hz}, 1 \mathrm{H}), 7.17(\mathrm{dd}, J=0.8,8.0 \mathrm{~Hz}, 1 \mathrm{H}), 7.05(\mathrm{dd}, J=1.2,7.6$ Hz, 1 H), 3.64 (s, 2 H), 3.26-3.12 (m, 1 H), 1.42 (s, 9 H), 1.39 (s, 12 H), 1.24 (d, J=6.8 Hz, 6 H); 13C NMR $\left(125 \mathrm{MHz}, \mathrm{CDCl}_{3}\right) \delta 171.2,153.3,138.2,129.4,127.1,122.6,83.8,80.4,42.6,33.3$, 28.1, 24.9, 24.4; HRMS-DART (m/z): calculated for $\mathrm{C}_{21} \mathrm{H}_{34} \mathrm{O}_{4} \mathrm{~B}[\mathrm{M}+\mathrm{H}]^{+} 361.2545$, found 361.2546 .<smiles>COc1ccccc1I</smiles><smiles>CC(C)(C)OC(=O)CCl</smiles>

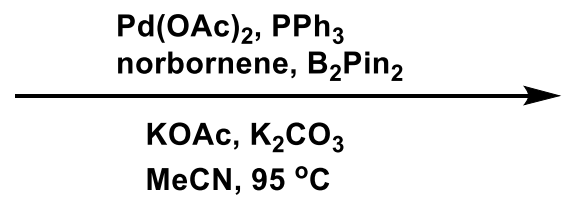

$\mathrm{MeCN}, 95^{\circ} \mathrm{C}$

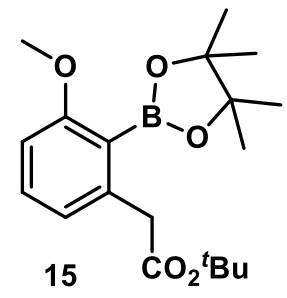

15

Compound 15 was synthesized following the General Procedure for the Alkylborylation of aryl iodidess. The reaction of 2-iodoanisole (37.4 mg, $0.160 \mathrm{mmol}, 1.0$ equiv.), 11 (96.0 mg, $0.640 \mathrm{mmol}, 4.0$ equiv.), $\mathrm{Pd}(\mathrm{OAc})_{2}\left(2.5 \mathrm{mg}, 0.0112 \mathrm{mmol}, 7 \%\right.$ ), $\mathrm{PPh}_{3}(5.9 \mathrm{mg}, 0.0224 \mathrm{mmol}$, $14 \%$ ), norbornene (22.6 mg, $0.240 \mathrm{mmol}, 1.5$ equiv.), $\mathrm{K}_{2} \mathrm{CO}_{3}$ (66.2 mg, $0.480 \mathrm{mmol}, 3.0$ equiv.), KOAc (102 mg, $1.04 \mathrm{mmol}, 6.5$ equiv.) and $\mathrm{B}_{2} \mathrm{pin}_{2}(81.3 \mathrm{mg}, 0.320 \mathrm{mmol}, 2.0$ equiv.) in $\mathrm{MeCN}$ 
$(5.0 \mathrm{~mL})$ delivered $15(26.2 \mathrm{mg}, 66 \%)$ after purification via silica gel column chromatography with $3 \%$ ethyl acetate/hexanes ( $\mathrm{R}_{\mathrm{f}}=0.3,5 \%$ ethyl acetate/hexanes). $1 \mathbf{H}$ NMR (400 MHz, $\left.\mathrm{CDCl}_{3}\right) \delta$ $7.26(\mathrm{t}, J=8.0 \mathrm{~Hz}, 1 \mathrm{H}), 6.85(\mathrm{~d}, J=7.6 \mathrm{~Hz}, 1 \mathrm{H}), 6.72(\mathrm{~d}, J=8.4 \mathrm{~Hz}, 1 \mathrm{H}), 3.78(\mathrm{~s}, 3 \mathrm{H}), 3.58$ (s, $2 \mathrm{H}), 1.42$ (s, $9 \mathrm{H}), 1.38$ (s, $12 \mathrm{H})$; 13C NMR (100 MHz, $\left.\mathrm{CDCl}_{3}\right) \delta$ 170.9, 163.2, 140.0, 130.6, 122.4, 108.4, 83.7, 80.4, 55.6, 42.5, 28.0, 24.8; HRMS-DART (m/z): calculated for $\mathrm{C}_{19} \mathrm{H}_{30} \mathrm{O}_{5} \mathrm{~B}$ $[\mathrm{M}+\mathrm{H}]^{+}$349.2181, found 349.2190.
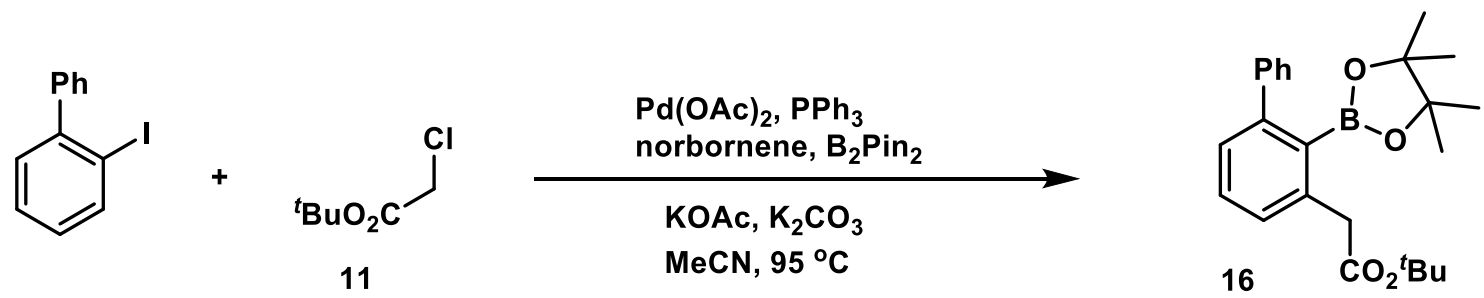

Compound 16 was synthesized following the General Procedure for the Alkylborylation of aryl iodidess. The reaction of 2-iodobiphenyl ( $44.8 \mathrm{mg}, 0.160 \mathrm{mmol}, 1.0$ equiv.), 11 (96.0 mg, $0.640 \mathrm{mmol}, 4.0$ equiv.), $\mathrm{Pd}(\mathrm{OAc})_{2}\left(2.5 \mathrm{mg}, 0.0112 \mathrm{mmol}, 7 \%\right.$ ), $\mathrm{PPh}_{3}(5.9 \mathrm{mg}, 0.0224 \mathrm{mmol}$, $14 \%$ ), norbornene (22.6 mg, $0.240 \mathrm{mmol}, 1.5$ equiv.), $\mathrm{K}_{2} \mathrm{CO}_{3}$ (66.2 mg, $0.480 \mathrm{mmol}, 3.0$ equiv.), KOAc (102 mg, $1.04 \mathrm{mmol}, 6.5$ equiv.) and $\mathrm{B}_{2} \operatorname{pin}_{2}(81.3 \mathrm{mg}, 0.320 \mathrm{mmol}, 2.0$ equiv.) in $\mathrm{MeCN}$ $(5.0 \mathrm{~mL})$ delivered $16(51.1 \mathrm{mg}, 81 \%)$ after purification via silica gel column chromatography with $2.5 \%$ ethyl acetate/hexanes ( $\mathrm{R}_{\mathrm{f}}=0.35,5 \%$ ethyl acetate/hexanes). $\mathbf{1 H} \mathbf{~ N M R}\left(400 \mathrm{MHz}, \mathrm{CDCl}_{3}\right)$ $\delta$ 7.43-7.18 (m, $8 \mathrm{H}), 3.71$ (s, $2 \mathrm{H}), 1.44(\mathrm{~s}, 9 \mathrm{H}), 1.14$ (s, $12 \mathrm{H}) ; \mathbf{1 3 C}$ NMR $\left(100 \mathrm{MHz}, \mathrm{CDCl}_{3}\right) \delta$ 171.0, 147.1, 143.8, 138.4, 129.2, 128.9, 128.3, 127.8, 127.3, 126.8, 83.8, 80.5, 42.4, 28.1, 24.9; HRMS-DART (m/z): calculated for $\mathrm{C}_{24} \mathrm{H}_{32} \mathrm{O}_{4} \mathrm{~B}[\mathrm{M}+\mathrm{H}]^{+}$395.2388, found 395.2390.
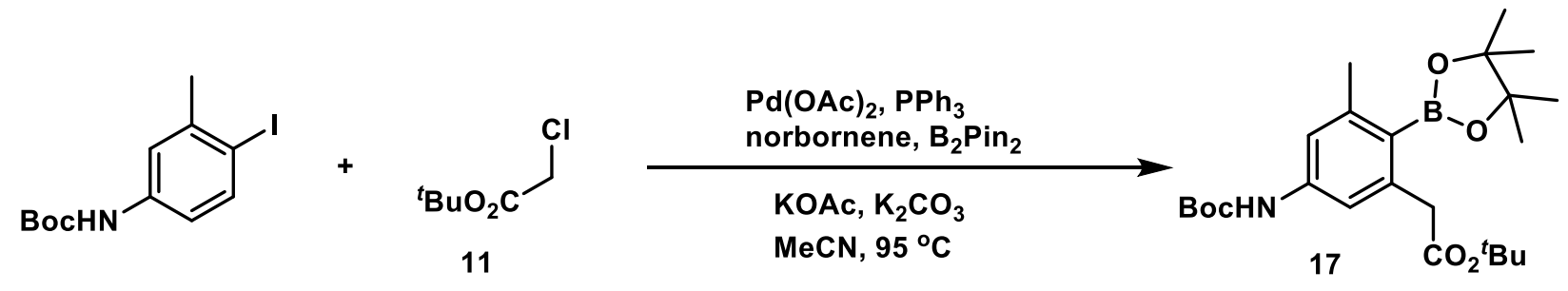

Compound 17 was synthesized following the General Procedure for the Alkylborylation of aryl iodidess. The reaction of tert-Butyl 4-iodo-3-methylphenylcarbamate (53.3 mg, 0.160 
mmol, 1.0 equiv.), 11 (96.0 mg, 0.640 mmol, 4.0 equiv.), $\mathrm{Pd}(\mathrm{OAc})_{2}(2.5 \mathrm{mg}, 0.0112 \mathrm{mmol}, 7$ \%), $\mathrm{PPh}_{3}$ (5.9 mg, $0.0224 \mathrm{mmol}, 14 \%$ ), norbornene (22.6 mg, $0.240 \mathrm{mmol}, 1.5$ equiv.), $\mathrm{K}_{2} \mathrm{CO}_{3}(66.2$ mg, 0.480 mmol, 3.0 equiv.), KOAc (102 mg, 1.04 mmol, 6.5 equiv.) and $B_{2}$ pin 2 (81.3 mg, 0.320 mmol, 2.0 equiv.) in $\mathrm{MeCN}(5.0 \mathrm{~mL})$ delivered 17 (44.4 mg, 62\%) after purification via silica gel column chromatography with $5 \%$ ethyl acetate/hexanes ( $\mathrm{R}_{\mathrm{f}}=0.3,10 \%$ ethyl acetate/hexanes $) . \mathbf{1 H}$ NMR (400 MHz, $\left.\mathrm{CDCl}_{3}\right) \delta 7.09$ (s, $\left.1 \mathrm{H}\right), 7.02$ (s, $\left.1 \mathrm{H}\right), 6.40$ (br, $\left.1 \mathrm{H}\right), 3.67$ (s, $\left.2 \mathrm{H}\right), 2.44$ (s, 3 H), $1.50(\mathrm{~s}, 9 \mathrm{H}), 1.43$ (s, $9 \mathrm{H}), 1.35$ (s, $12 \mathrm{H}) ; 13 \mathrm{C}$ NMR $\left(100 \mathrm{MHz}, \mathrm{CDCl}_{3}\right) \delta 171.4,152.4,144.9$, 140.9, 139.3, 117.8, 117.0, 83.4, 80.3, 42.5, 28.3, 28.2, 24.9, 22.8; HRMS-DART (m/z): calculated for $\mathrm{C}_{24} \mathrm{H}_{39} \mathrm{O}_{6} \mathrm{NB}[\mathrm{M}+\mathrm{H}]^{+} 448.2865$, found 448.2870.
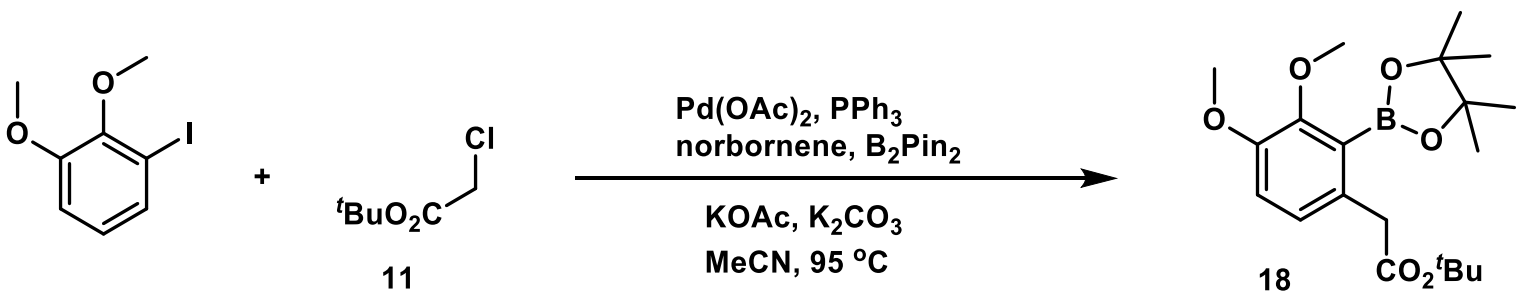

Compound 18 was synthesized following the General Procedure for the Alkylborylation of aryl iodidess. The reaction of 1-iodo-2,3-dimethoxybenzene (42.2 mg, $0.160 \mathrm{mmol}, 1.0$ equiv.), 11 (96.0 mg, 0.640 mmol, 4.0 equiv.), $\mathrm{Pd}(\mathrm{OAc})_{2}\left(2.5 \mathrm{mg}, 0.0112 \mathrm{mmol}, 7\right.$ \%), $\mathrm{PPh}_{3}(5.9 \mathrm{mg}, 0.0224$ mmol, 14 \%), norbornene (22.6 mg, $0.240 \mathrm{mmol}, 1.5$ equiv.), $\mathrm{K}_{2} \mathrm{CO}_{3}$ (66.2 mg, $0.480 \mathrm{mmol}, 3.0$ equiv.), KOAc (102 mg, $1.04 \mathrm{mmol}, 6.5$ equiv.) and $\mathrm{B}_{2} \operatorname{pin}_{2}(81.3 \mathrm{mg}, 0.320 \mathrm{mmol}, 2.0$ equiv.) in $\mathrm{MeCN}(5.0 \mathrm{~mL})$ delivered $18(30.9 \mathrm{mg}, 51 \%)$ after purification via silica gel column chromatography with $8 \%$ ethyl acetate/hexanes ( $R_{f}=0.3,15 \%$ ethyl acetate/hexanes). 1H NMR $\left(500 \mathrm{MHz}, \mathrm{CDCl}_{3}\right) \delta 6.93(\mathrm{~d}, J=8.0 \mathrm{~Hz}, 1 \mathrm{H}), 6.86(\mathrm{~d}, J=8.0 \mathrm{~Hz}, 1 \mathrm{H}), 3.83(\mathrm{~s}, 3 \mathrm{H}), 3.82(\mathrm{~s}, 3$ H), 3.53 (s, $2 \mathrm{H}), 1.42$ (s, $9 \mathrm{H}), 1.37$ (s, $12 \mathrm{H})$; 13C NMR (125 MHz, $\left.\mathrm{CDCl}_{3}\right) \delta 171.3,152.4,151.0$, 131.0, 125.9, 113.9, 83.8, 80.4, 61.4, 55.8, 41.5, 28.1, 24.8; HRMS-DART (m/z): calculated for $\mathrm{C}_{20} \mathrm{H}_{32} \mathrm{O}_{6} \mathrm{~B}[\mathrm{M}+\mathrm{H}]^{+}$379.2286, found 379.2283. 
<smiles>Clc1ccccc1I</smiles>

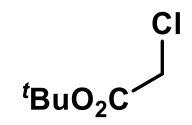

11

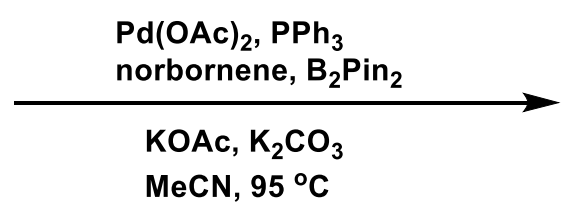

$\mathrm{KOAc}, \mathrm{K}_{2} \mathrm{CO}_{3}$

$\mathrm{CN}, 95^{\circ} \mathrm{C}$

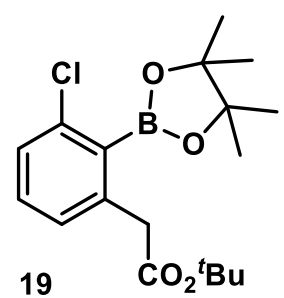

Compound 19 was synthesized following the General Procedure for the Alkylborylation of aryl iodidess. The reaction of 1-chloro-2-iodobenzene ( $38.1 \mathrm{mg}, 0.160 \mathrm{mmol}, 1.0$ equiv.), 11 (96.0 mg, 0.640 mmol, 4.0 equiv.), $\mathrm{Pd}(\mathrm{OAc})_{2}$ (2.5 mg, $0.0112 \mathrm{mmol}, 7$ \%), $\mathrm{PPh}_{3}$ (5.9 mg, 0.0224 mmol, $14 \%$ ), norbornene (22.6 mg, $0.240 \mathrm{mmol}, 1.5$ equiv.), $\mathrm{K}_{2} \mathrm{CO}_{3}$ (66.2 mg, $0.480 \mathrm{mmol}, 3.0$ equiv.), KOAc (102 mg, $1.04 \mathrm{mmol}, 6.5$ equiv.) and $\mathrm{B}_{2} \mathrm{pin}_{2}(81.3 \mathrm{mg}, 0.320 \mathrm{mmol}, 2.0$ equiv.) in $\mathrm{MeCN}(5.0 \mathrm{~mL})$ delivered $19(29.9 \mathrm{mg}, 53 \%)$ after purification via silica gel column chromatography with $3 \%$ ethyl acetate/hexanes ( $\mathrm{R}_{\mathrm{f}}=0.35,5 \%$ ethyl acetate/hexanes). 1H NMR $\left(400 \mathrm{MHz}, \mathrm{CDCl}_{3}\right) \delta$ 7.25-7.20 (m, $\left.2 \mathrm{H}\right), 7.13$ (dd, $\left.J=2.4,6.4 \mathrm{~Hz}, 1 \mathrm{H}\right), 3.60(\mathrm{~s}, 2 \mathrm{H}), 1.42(\mathrm{~s}, 9$ $\mathrm{H}), 1.41$ (s, $12 \mathrm{H}) ; 13 \mathrm{C}$ NMR $\left(100 \mathrm{MHz}, \mathrm{CDCl}_{3}\right) \delta 170.4,140.3,138.1,130.4,127.9,127.2,84.5$, 80.9, 42.4, 28.1, 24.9; HRMS-DART (m/z): calculated for $\mathrm{C}_{18} \mathrm{H}_{27} \mathrm{ClO}_{4} \mathrm{~B}[\mathrm{M}+\mathrm{H}]^{+} 353.1685$, found 353.1690 .<smiles>Cc1c(Cl)cccc1I</smiles>

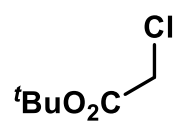

11

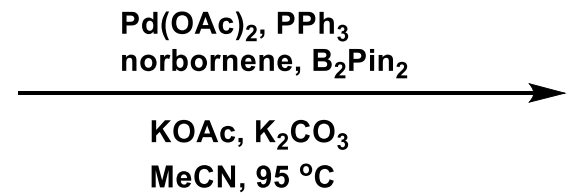

$\mathrm{MeCN}, 95^{\circ} \mathrm{C}$

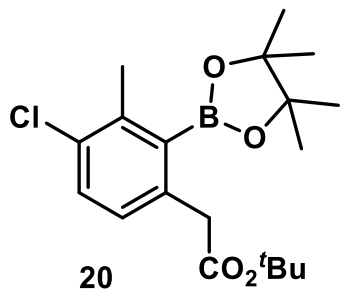

20

Compound 20 was synthesized following the General Procedure for the Alkylborylation of aryl iodides. The reaction of 2-chloro-6-iodotoluene (40.3mg, $0.160 \mathrm{mmol}, 1.0$ equiv.), 11 (96.0 mg, 0.640 mmol, 4.0 equiv.), $\mathrm{Pd}(\mathrm{OAc})_{2}\left(2.5 \mathrm{mg}, 0.0112 \mathrm{mmol}, 7\right.$ \%), $\mathrm{PPh}_{3}(5.9 \mathrm{mg}, 0.0224 \mathrm{mmol}$, $14 \%$ ), norbornene (22.6 mg, $0.240 \mathrm{mmol}, 1.5$ equiv.), $\mathrm{K}_{2} \mathrm{CO}_{3}$ (66.2 mg, $0.480 \mathrm{mmol}, 3.0$ equiv.), KOAc (102 mg, $1.04 \mathrm{mmol}, 6.5$ equiv.) and $\mathrm{B}_{2} \mathrm{pin}_{2}$ (81.3 mg, $0.320 \mathrm{mmol}, 2.0$ equiv.) in MeCN $(5.0 \mathrm{~mL})$ delivered $\mathbf{2 0}(38.1 \mathrm{mg}, 65 \%)$ after purification via silica gel column chromatography with $3 \%$ ethyl acetate/hexanes $\left(\mathrm{R}_{\mathrm{f}}=0.35,5 \%\right.$ ethyl acetate/hexanes $) .1 \mathbf{H}$ NMR $\left(400 \mathrm{MHz}, \mathrm{CDCl}_{3}\right) \delta$ $7.29(\mathrm{~d}, J=8.0 \mathrm{~Hz}, 1 \mathrm{H}), 6.99$ (d, $J=8.4 \mathrm{~Hz}, 1 \mathrm{H}), 3.62$ (s, $2 \mathrm{H}), 2.47$ (s, $3 \mathrm{H}), 1.42(\mathrm{~s}, 9 \mathrm{H}), 1.39$ 
(s, $12 \mathrm{H}) ; 13 \mathrm{C}$ NMR (100 MHz, $\left.\mathrm{CDCl}_{3}\right) \delta$ 170.9, 139.7, 137.4, 133.3, 130.1, 128.7, 84.1, 80.6, 42.1, 28.1, 25.0, 19.8; HRMS-DART (m/z): calculated for $\mathrm{C}_{19} \mathrm{H}_{29} \mathrm{ClO}_{4} \mathrm{~B}[\mathrm{M}+\mathrm{H}]^{+} 367.1842$, found 367.1851 .

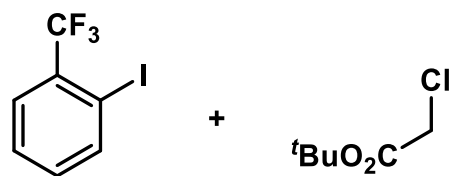

11

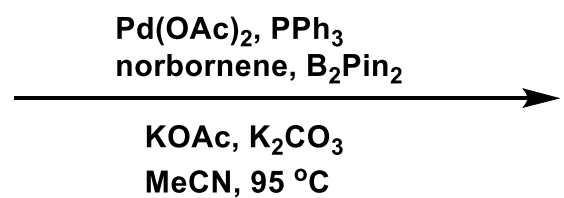

$\mathrm{MeCN}, 95^{\circ} \mathrm{C}$

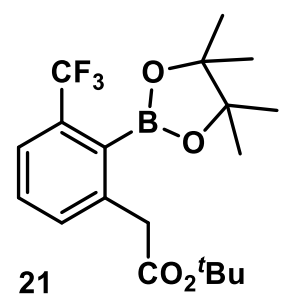

Compound 21 was synthesized following the General Procedure for the Alkylborylation of aryl iodides. The reaction of 2-iodobenzotrifluoride (43.5 mg, $0.160 \mathrm{mmol}, 1.0$ equiv.), 11 (96.0 mg, 0.640 mmol, 4.0 equiv.), $\mathrm{Pd}(\mathrm{OAc})_{2}\left(2.5 \mathrm{mg}, 0.0112 \mathrm{mmol}, 7\right.$ \%), $\mathrm{PPh}_{3}(5.9 \mathrm{mg}, 0.0224 \mathrm{mmol}$, $14 \%$ ), norbornene (22.6 mg, $0.240 \mathrm{mmol}, 1.5$ equiv.), $\mathrm{K}_{2} \mathrm{CO}_{3}$ (66.2 mg, $0.480 \mathrm{mmol}, 3.0$ equiv.), KOAc (102 mg, 1.04 mmol, 6.5 equiv.) and $\mathrm{B}_{2} \mathrm{pin}_{2}$ (81.3 mg, $0.320 \mathrm{mmol}, 2.0$ equiv.) in MeCN $(5.0 \mathrm{~mL})$ delivered $21(32.1 \mathrm{mg}, 52 \%)$ after purification via silica gel column chromatography with $3.5 \%$ ethyl acetate/hexanes ( $\mathrm{R}_{\mathrm{f}}=0.3,5 \%$ ethyl acetate/hexanes). $1 \mathbf{H} \mathbf{N M R}\left(400 \mathrm{MHz}, \mathrm{CDCl}_{3}\right) \delta$ 7.54 (dd, $J=5.6,8.4$ Hz, 1 H), 7.46-7.38 (m, 2 H), 3.68 (s, 2 H), 1.43 (s, 9 H), 1.40 (s, 12 H); 13C NMR (125 MHz, $\left.\mathrm{CDCl}_{3}\right) \delta 170.2,139.2,133.7$ (q, $\left.J=30.0 \mathrm{~Hz}\right), 133.1,129.2,124.6(\mathrm{~d}, J=266$ $\mathrm{Hz}), 123.8$ (q, $J=3.8 \mathrm{~Hz}), 84.6,81.0,42.2,28.0,25.0 ;$ 19F NMR (376 $\left.\mathrm{MHz}, \mathrm{CDCl}_{3}\right) \delta-58.9$; HRMS-DART (m/z): calculated for $\mathrm{C}_{19} \mathrm{H}_{27} \mathrm{~F}_{3} \mathrm{O}_{4} \mathrm{~B}[\mathrm{M}+\mathrm{H}]^{+}$387.1949, found 387.1952.
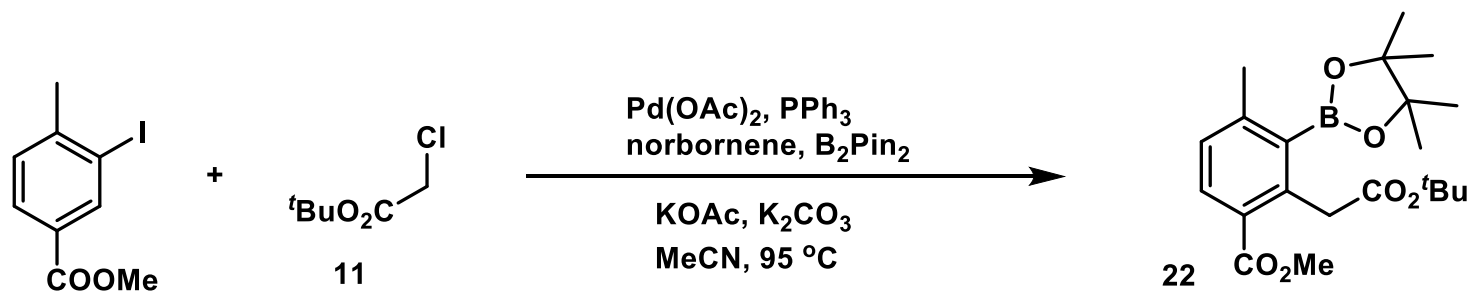

Compound 22 was synthesized following the General Procedure for the Alkylborylation of aryl iodides. The reaction of Methyl-3-iodo-4-methylbenzoate (44.2 mg, $0.160 \mathrm{mmol}, 1.0$ equiv.), 11 (96.0 mg, 0.640 mmol, 4.0 equiv.), $\mathrm{Pd}(\mathrm{OAc})_{2}$ (2.5 mg, $0.0112 \mathrm{mmol}, 7$ \%), $\mathrm{PPh}_{3}$ (5.9 mg, $0.0224 \mathrm{mmol}, 14 \%$ ), norbornene (22.6 mg, $0.240 \mathrm{mmol}, 1.5$ equiv.), $\mathrm{K}_{2} \mathrm{CO}_{3}$ (66.2 mg, 0.480 mmol, 3.0 equiv.), KOAc (102 mg, 1.04 mmol, 6.5 equiv.) and $B_{2} \operatorname{pin}_{2}(81.3 \mathrm{mg}, 0.320 \mathrm{mmol}, 2.0$ 
equiv.) in $\mathrm{MeCN}(5.0 \mathrm{~mL})$ delivered $22(18.1 \mathrm{mg}, 29 \%)$ after purification via silica gel column chromatography with $3 \%$ ethyl acetate/hexanes $\left(\mathrm{R}_{\mathrm{f}}=0.25,5 \%\right.$ ethyl acetate/hexanes $)$. 1H NMR $\left(400 \mathrm{MHz}, \mathrm{CDCl}_{3}\right) \delta 7.84(\mathrm{~d}, J=8.0 \mathrm{~Hz}, 1 \mathrm{H}), 7.09$ (d, $\left.J=8.0 \mathrm{~Hz}, 1 \mathrm{H}\right), 4.06(\mathrm{~s}, 2 \mathrm{H}), 3.84(\mathrm{~s}, 3$ H), 2.45 (s, $3 \mathrm{H}), 1.42$ (s, $9 \mathrm{H}), 1.39$ (s, $12 \mathrm{H})$; 13C NMR (100 MHz, CDCl 3 ) $\delta 170.6,168.1,146.5$, 140.0, 131.8, 128.0, 127.4, 84.1, 80.1, 51.7, 40.6, 28.1, 25.0, 22.5; HRMS-DART (m/z): calculated for $\mathrm{C}_{21} \mathrm{H}_{32} \mathrm{O}_{6} \mathrm{~B}[\mathrm{M}+\mathrm{H}]^{+}$391.2286, found 391.2293.
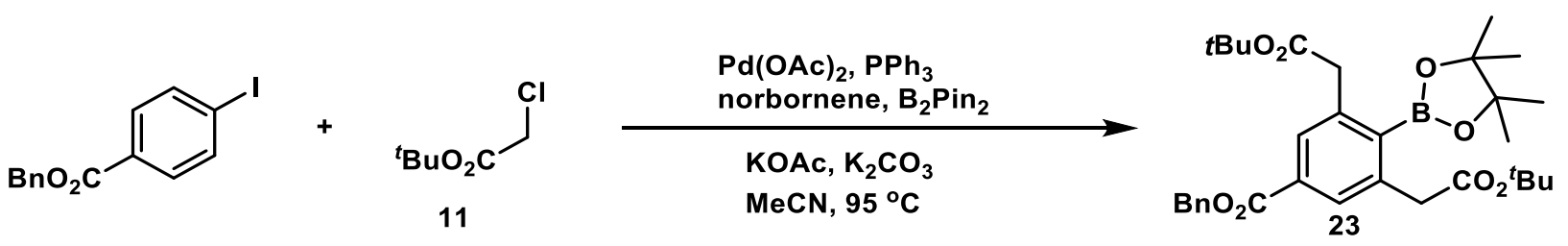

Compound 23 was synthesized following the General Procedure for the Alkylborylation of aryl iodides. The reaction of benzyl 4-iodobenzoate (54.1 mg, $0.160 \mathrm{mmol}, 1.0$ equiv.), 11 (96.0 mg, 0.640 mmol, 4.0 equiv.), $\mathrm{Pd}(\mathrm{OAc})_{2}\left(2.5 \mathrm{mg}, 0.0112 \mathrm{mmol}, 7\right.$ \%), $\mathrm{PPh}_{3}(5.9 \mathrm{mg}, 0.0224 \mathrm{mmol}$, $14 \%$ ), norbornene (22.6 mg, $0.240 \mathrm{mmol}, 1.5$ equiv.), $\mathrm{K}_{2} \mathrm{CO}_{3}$ (66.2 mg, $0.480 \mathrm{mmol}, 3.0$ equiv.), KOAc (102 mg, $1.04 \mathrm{mmol}, 6.5$ equiv.) and $\mathrm{B}_{2} \mathrm{pin}_{2}$ (81.3 mg, $0.320 \mathrm{mmol}, 2.0$ equiv.) in $\mathrm{MeCN}$ $(5.0 \mathrm{~mL})$ delivered $\mathbf{2 3}(39.9 \mathrm{mg}, 44 \%)$ after purification via silica gel column chromatography with $10 \%$ ethyl acetate/hexanes $\left(\mathrm{R}_{\mathrm{f}}=0.35,10 \%\right.$ ethyl acetate/hexanes $)$. $1 \mathbf{H} \mathbf{N M R}\left(400 \mathrm{MHz}, \mathrm{CDCl}_{3}\right)$ $\delta 7.78$ (s, 2 H), 7.48-7.30 (m, 5 H), 5.34 (s, 2 H), 3.87 (s, 4 H), 1.42 (s, 18 H), 1.36 (s, 12 H); 13C NMR $\left(100 \mathrm{MHz}, \mathrm{CDCl}_{3}\right) \delta 171.1,166.3,140.5,136.0,130.8,129.6,128.6,128.3,128.2,83.9$, 80.6, 66.7, 42.3, 28.1, 25.0; HRMS-DART (m/z): calculated for $\mathrm{C}_{32} \mathrm{H}_{44} \mathrm{O}_{8} \mathrm{~B}[\mathrm{M}+\mathrm{H}]^{+}$567.3124, found 567.3125 .
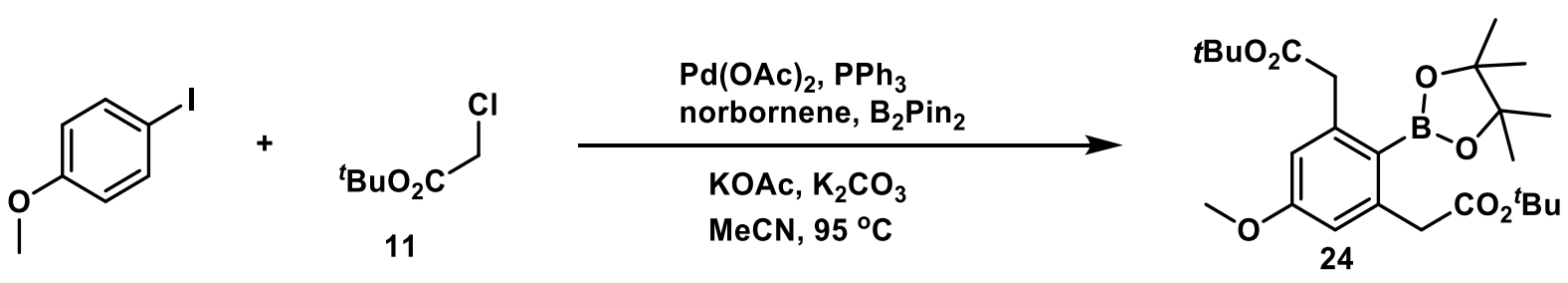

Compound 24 was synthesized following the General Procedure for the Alkylborylation of aryl iodides. The reaction of 4-iodoanisole (54.1 mg, $0.160 \mathrm{mmol}, 1.0$ equiv.), 11 (96.0 mg, 
$0.640 \mathrm{mmol}, 4.0$ equiv.), $\mathrm{Pd}(\mathrm{OAc})_{2}$ (2.5 mg, $0.0112 \mathrm{mmol}, 7 \%$ \%), $\mathrm{PPh}_{3}(5.9 \mathrm{mg}, 0.0224 \mathrm{mmol}$, $14 \%$ ), norbornene (22.6 mg, $0.240 \mathrm{mmol}, 1.5$ equiv.), $\mathrm{K}_{2} \mathrm{CO}_{3}$ (66.2 mg, $0.480 \mathrm{mmol}, 3.0$ equiv.), KOAc (102 mg, $1.04 \mathrm{mmol}, 6.5$ equiv.) and $\mathrm{B}_{2} \mathrm{pin}_{2}(81.3 \mathrm{mg}, 0.320 \mathrm{mmol}, 2.0$ equiv.) in $\mathrm{MeCN}$ $(5.0 \mathrm{~mL})$ delivered $24(38.5 \mathrm{mg}, 52 \%)$ after purification via silica gel column chromatography with $8 \%$ ethyl acetate/hexanes ( $\mathrm{R}_{\mathrm{f}}=0.35,10 \%$ ethyl acetate/hexanes). $1 \mathbf{H} \mathbf{~ N M R}\left(400 \mathrm{MHz}, \mathrm{CDCl}_{3}\right) \delta$ 6.65 (s, 2 H), 3.81 (s, 4 H), 3.78 (s, 3 H), 1.42 (s, 18 H), 1.33 (s, 12 H); 13C NMR (100 MHz, $\left.\mathrm{CDCl}_{3}\right) \delta 171.5,160.4,142.4,114.6,83.3,80.2,55.0,42.8,28.2,24.9 ;$ HRMS-DART (m/z): calculated for $\mathrm{C}_{25} \mathrm{H}_{40} \mathrm{O}_{6} \mathrm{~B}[\mathrm{M}+\mathrm{H}]^{+} 463.2862$, found 463.2857 .

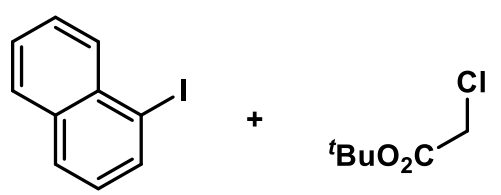

11

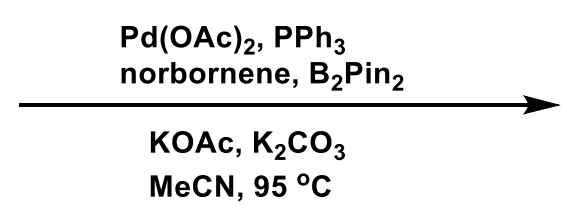

$\mathrm{MeCN}, 95^{\circ} \mathrm{C}$

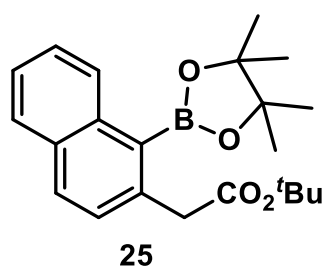

25

Compound 25 was synthesized following the General Procedure for the Alkylborylation of aryl iodides. The reaction of 1-iodonaphthalene (40.6 mg, $0.160 \mathrm{mmol}, 1.0$ equiv.), 11 (96.0 mg, 0.640 mmol, 4.0 equiv.), $\mathrm{Pd}(\mathrm{OAc})_{2}$ (2.5 mg, $0.0112 \mathrm{mmol}, 7$ \%), $\mathrm{PPh}_{3}(5.9 \mathrm{mg}, 0.0224 \mathrm{mmol}$, $14 \%$ ), norbornene (22.6 mg, $0.240 \mathrm{mmol}, 1.5$ equiv.), $\mathrm{K}_{2} \mathrm{CO}_{3}$ (66.2 mg, $0.480 \mathrm{mmol}, 3.0$ equiv.), KOAc (102 mg, $1.04 \mathrm{mmol}, 6.5$ equiv.) and $\mathrm{B}_{2} \mathrm{pin}_{2}$ (81.3 mg, $0.320 \mathrm{mmol}, 2.0$ equiv.) in $\mathrm{MeCN}$ $(5.0 \mathrm{~mL})$ delivered $25(40.6 \mathrm{mg}, 69 \%)$ after purification via silica gel column chromatography with $2.5 \%$ ethyl acetate/hexanes ( $\mathrm{R}_{\mathrm{f}}=0.35,5 \%$ ethyl acetate/hexanes). $\mathbf{1 H} \mathbf{~ N M R}\left(400 \mathrm{MHz}, \mathrm{CDCl}_{3}\right)$ $\delta 8.32(\mathrm{~d}, J=8.4 \mathrm{~Hz}, 1 \mathrm{H}), 7.82(\mathrm{~d}, J=8.4 \mathrm{~Hz}, 1 \mathrm{H}), 7.79(\mathrm{~d}, J=7.6 \mathrm{~Hz}, 1 \mathrm{H}), 7.52-7.33(\mathrm{~m}, 3 \mathrm{H})$, 3.93 (s, $2 \mathrm{H}), 1.48$ (s, $12 \mathrm{H}), 1.43$ (s, $9 \mathrm{H})$; 13C NMR (100 MHz, CDCl 3 ) $\delta$ 171.1, 138.7, 136.8, 132.0, 128.1, 126.1, 125.2, 84.0, 80.5, 43.0, 28.1, 25.0; HRMS-DART $(\mathrm{m} / \mathrm{z})$ : calculated for $\mathrm{C}_{22} \mathrm{H}_{30} \mathrm{O} 4 \mathrm{~B}[\mathrm{M}+\mathrm{H}]^{+}$369.2232, found 369.2237.

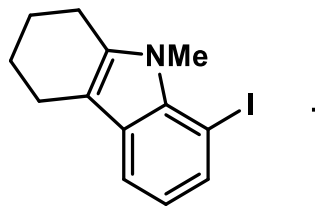

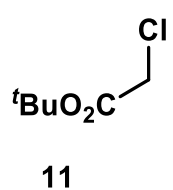

11

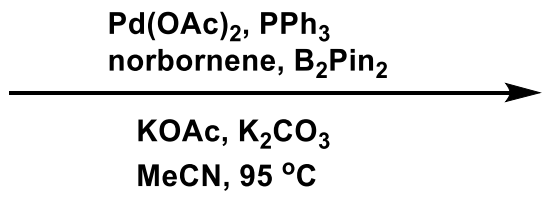

$\mathrm{MeCN}, 95^{\circ} \mathrm{C}$

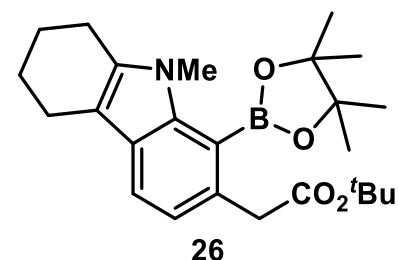

26 
Compound 26 was synthesized following the General Procedure for the Alkylborylation of aryl iodides. The reaction of 1,2,3,4-tetrahydro-8-iodo-9-methylcarbazole (49.8 mg, 0.160 mmol, 1.0 equiv.), 11 (96.0 mg, 0.640 mmol, 4.0 equiv.), $\mathrm{Pd}(\mathrm{OAc})_{2}$ (2.5 mg, $0.0112 \mathrm{mmol}, 7$ \%), $\mathrm{PPh}_{3}$ (5.9 mg, $0.0224 \mathrm{mmol}, 14 \%$ ), norbornene (22.6 mg, $0.240 \mathrm{mmol}, 1.5$ equiv.), $\mathrm{K}_{2} \mathrm{CO}_{3}(66.2$ mg, 0.480 mmol, 3.0 equiv.), KOAc (102 mg, 1.04 mmol, 6.5 equiv.) and $B_{2} p_{2}$ (81.3 mg, 0.320 mmol, 2.0 equiv.) in $\mathrm{MeCN}(5.0 \mathrm{~mL})$ delivered 26 (48.3 mg, 71\%) after purification via silica gel column chromatography with $3 \%$ ethyl acetate/hexanes $\left(R_{f}=0.3,5 \%\right.$ ethyl acetate/hexanes $) . .1 H$ NMR (400 MHz, $\left.\mathrm{CDCl}_{3}\right) \delta 7.42(\mathrm{~d}, J=8.0 \mathrm{~Hz}, 1 \mathrm{H}), 6.97$ (d, $\left.J=8.0 \mathrm{~Hz}, 1 \mathrm{H}\right), 3.81$ (s, $\left.2 \mathrm{H}\right), 3.67$ (s, 3 H), 2.74-2.60 (m, 4 H), 1.98-1.87 (m, 2 H), 1.87-1.76 (m, 2 H), 1.44 (s, 12 H), 1.42 (s, 9 H); 13C NMR $\left(100 \mathrm{MHz}, \mathrm{CDCl}_{3}\right) \delta 171.2,140.2,135.8,132.0,126.0,121.0,119.0,109.2,84.0,80.0$, 42.8, 31.5, 28.1, 25.4, 23.4, 23.2, 22.4, 20.9; HRMS-DART (m/z): calculated for $\mathrm{C}_{27} \mathrm{H}_{41} \mathrm{NO}_{4} \mathrm{~B}$ $[\mathrm{M}+\mathrm{H}]^{+} 426.2810$, found 426.2815 .
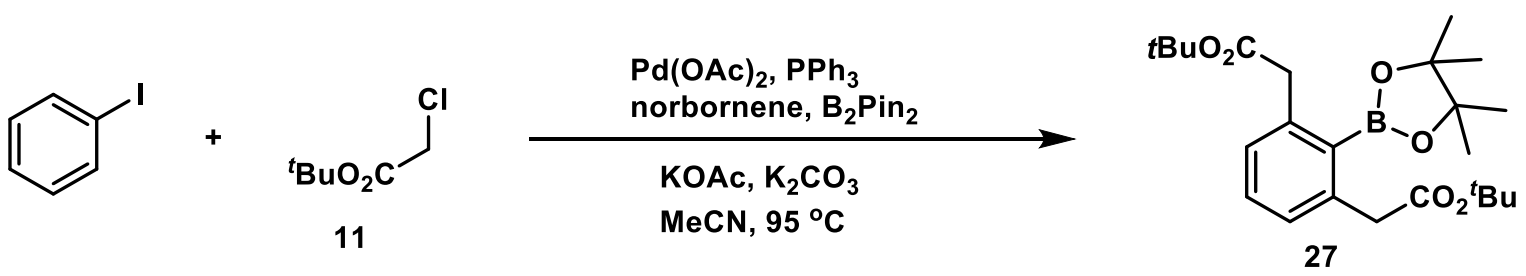

Compound 27 was synthesized following the General Procedure for the Alkylborylation of aryl iodides. The reaction of iodobenzene (32.6 mg, $0.160 \mathrm{mmol}, 1.0$ equiv.), 11 (96.0 mg, 0.640 mmol, 4.0 equiv.), $\mathrm{Pd}(\mathrm{OAc})_{2}\left(2.5 \mathrm{mg}, 0.0112 \mathrm{mmol}, 7 \%\right.$ ), $\mathrm{PPh}_{3}(5.9 \mathrm{mg}, 0.0224 \mathrm{mmol}$, $14 \%$ ), norbornene (22.6 mg, $0.240 \mathrm{mmol}, 1.5$ equiv.), $\mathrm{K}_{2} \mathrm{CO}_{3}$ (66.2 mg, $0.480 \mathrm{mmol}, 3.0$ equiv.), KOAc (102 mg, 1.04 mmol, 6.5 equiv.) and $\mathrm{B}_{2} \operatorname{pin}_{2}$ (81.3 mg, $0.320 \mathrm{mmol}, 2.0$ equiv.) in MeCN $(5.0 \mathrm{~mL})$ delivered $27(33.2 \mathrm{mg}, 48 \%)$ after purification via silica gel column chromatography with $5 \%$ ethyl acetate/hexanes $\left(\mathrm{R}_{\mathrm{f}}=0.5,10 \%\right.$ ethyl acetate/hexanes $) .1 \mathbf{H}$ NMR $\left(400 \mathrm{MHz}, \mathrm{CDCl}_{3}\right) \delta$ $7.28(\mathrm{t}, J=7.6 \mathrm{~Hz}, 1 \mathrm{H}), 7.11(\mathrm{~d}, J=7.6 \mathrm{~Hz}, 2 \mathrm{H}), 3.82(\mathrm{~s}, 4 \mathrm{H}), 1.44(\mathrm{~s}, 18 \mathrm{H}), 1.38$ (s, $12 \mathrm{H})$; 13C NMR $\left(100 \mathrm{MHz}, \mathrm{CDCl}_{3}\right) \delta 171.6,140.1,129.6,128.7,83.6,80.2,42.6,28.1,25.0$; HRMSDART (m/z): calculated for $\mathrm{C}_{24} \mathrm{H}_{38} \mathrm{O}_{6} \mathrm{~B}[\mathrm{M}+\mathrm{H}]^{+} 433.2756$, found 433.2750. 


\section{The Scope of Alkyl halides}
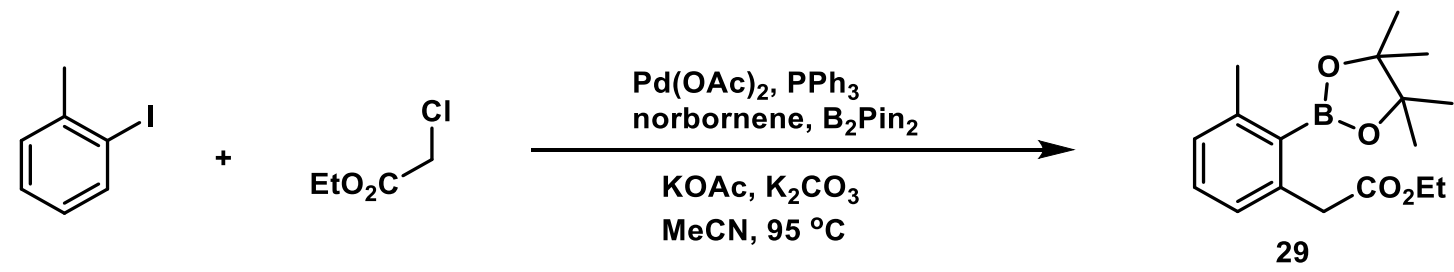

Compound 29 was synthesized following the General Procedure for the Alkylborylation of aryl iodides. The reaction of 2-iodotoluene (34.9 $\mathrm{mg}, 0.160 \mathrm{mmol}, 1.0$ equiv.), ethyl chloroacetate (78.1 mg, $0.640 \mathrm{mmol}, 4.0$ equiv.), $\mathrm{Pd}(\mathrm{OAc})_{2}$ (2.5 mg, $0.0112 \mathrm{mmol}, 7 \%$ ), $\mathrm{PPh}_{3}(5.9$ $\mathrm{mg}, 0.0224 \mathrm{mmol}, 14 \%$ ), norbornene (22.6 mg, $0.240 \mathrm{mmol}, 1.5$ equiv.), $\mathrm{K}_{2} \mathrm{CO}_{3}$ (66.2 mg, 0.480 mmol, 3.0 equiv.), KOAc (102 mg, $1.04 \mathrm{mmol}, 6.5$ equiv.) and $\mathrm{B}_{2} \mathrm{pin}_{2}(81.3 \mathrm{mg}, 0.320 \mathrm{mmol}, 2.0$ equiv.) in $\mathrm{MeCN}(5.0 \mathrm{~mL})$ delivered 29 (31.1 mg, 64\%) after purification via silica gel column chromatography with $2.5 \%$ ethyl acetate/hexanes $\left(R_{\mathrm{f}}=0.4,5 \%\right.$ ethyl acetate/hexanes). 1 H NMR $\left(600 \mathrm{MHz}, \mathrm{CDCl}_{3}\right) \delta 7.20(\mathrm{t}, J=7.2 \mathrm{~Hz}, 1 \mathrm{H}), 7.04(\mathrm{t}, J=8.4 \mathrm{~Hz}, 2 \mathrm{H}), 4.12(\mathrm{q}, J=6.6 \mathrm{~Hz}, 2 \mathrm{H})$, 3.79 (s, $2 \mathrm{H}), 2.46$ (s, $3 \mathrm{H}), 1.37$ (s, $12 \mathrm{H}), 1.24$ (t, $J=6.6 \mathrm{~Hz}, 3 \mathrm{H}) ; \mathbf{1 3 C} \mathbf{N M R}\left(150 \mathrm{MHz}, \mathrm{CDCl}_{3}\right)$ $\delta$ 172.2 $143.3,138.9,129.6,128.4,127.2,83.7,60.6,41.6,25.0,22.6,14.2 ;$ HRMS-DART (m/z): calculated for $\mathrm{C}_{17} \mathrm{H}_{26} \mathrm{O}_{4} \mathrm{~B}[\mathrm{M}+\mathrm{H}]^{+}$305.1919, found 305.1922.
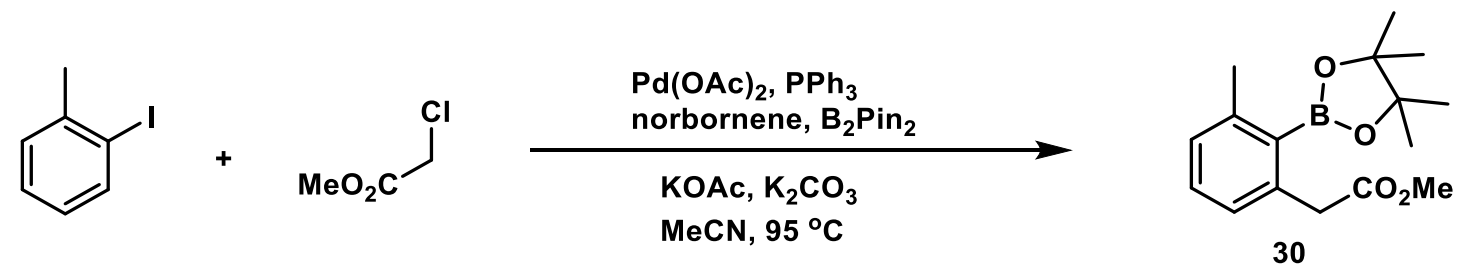

Compound $\mathbf{3 0}$ was synthesized following the General Procedure for the Alkylborylation of aryl iodides. The reaction of 2-iodotoluene (34.9 $\mathrm{mg}, 0.160 \mathrm{mmol}, 1.0$ equiv.), methyl chloroacetate (69.1 mg, $0.640 \mathrm{mmol}, 4.0$ equiv.), $\mathrm{Pd}(\mathrm{OAc})_{2}$ (2.5 mg, $0.0112 \mathrm{mmol}, 7 \%$ ), $\mathrm{PPh}_{3}(5.9$ $\mathrm{mg}, 0.0224 \mathrm{mmol}, 14 \%$ ), norbornene (22.6 mg, $0.240 \mathrm{mmol}, 1.5$ equiv.), $\mathrm{K}_{2} \mathrm{CO}_{3}(66.2 \mathrm{mg}, 0.480$ 
mmol, 3.0 equiv.), KOAc (102 mg, $1.04 \mathrm{mmol}, 6.5$ equiv.) and $\mathrm{B}_{2} \mathrm{pin}_{2}(81.3 \mathrm{mg}, 0.320 \mathrm{mmol}, 2.0$ equiv.) in $\mathrm{MeCN}(5.0 \mathrm{~mL})$ delivered 30 (26.4 $\mathrm{mg}, 57 \%)$ after purification via silica gel column chromatography with $2.5 \%$ ethyl acetate/hexanes $\left(R_{\mathrm{f}}=0.4,5 \%\right.$ ethyl acetate/hexanes). 1 H NMR $\left(500 \mathrm{MHz}, \mathrm{CDCl}_{3}\right) \delta 7.21(\mathrm{t}, J=8.0 \mathrm{~Hz}, 1 \mathrm{H}), 7.06(\mathrm{~d}, J=8.0 \mathrm{~Hz}, 1 \mathrm{H}), 7.02(\mathrm{~d}, J=7.5 \mathrm{~Hz}, 1 \mathrm{H})$, 3.81 (s, $2 \mathrm{H}), 3.66$ (s, $3 \mathrm{H}), 2.47$ (s, $3 \mathrm{H}), 1.37$ (s, $12 \mathrm{H})$; 13C NMR $\left(125 \mathrm{MHz}, \mathrm{CDCl}_{3}\right) \delta$ 172.7, 143.4, 138.8, 129.7, 128.5, 127.3, 83.7, 51.8, 41.5, 25.0, 22.6; HRMS-DART (m/z): calculated for $\mathrm{C}_{16} \mathrm{H}_{24} \mathrm{O}_{4} \mathrm{~B}[\mathrm{M}+\mathrm{H}]^{+}$291.1762, found 291.1766.
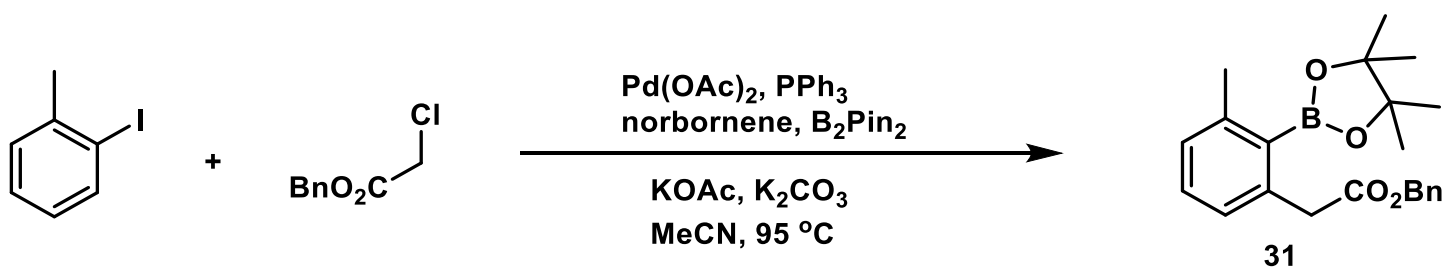

Compound 31 was synthesized following the General Procedure for the Alkylborylation of aryl iodides. The reaction of 2-iodotoluene (34.9 mg, $0.160 \mathrm{mmol}, 1.0$ equiv.), benzyl chloroacetate (118 mg, $0.640 \mathrm{mmol}, 4.0$ equiv.), $\mathrm{Pd}(\mathrm{OAc})_{2}$ (2.5 mg, $0.0112 \mathrm{mmol}, 7 \%$ ), $\mathrm{PPh}_{3}(5.9$ $\mathrm{mg}, 0.0224 \mathrm{mmol}, 14 \%$ ), norbornene (22.6 mg, $0.240 \mathrm{mmol}, 1.5$ equiv.), $\mathrm{K}_{2} \mathrm{CO}_{3}$ (66.2 mg, 0.480 mmol, 3.0 equiv.), KOAc (102 mg, $1.04 \mathrm{mmol}, 6.5$ equiv.) and $\mathrm{B}_{2} \mathrm{pin}_{2}(81.3 \mathrm{mg}, 0.320 \mathrm{mmol}, 2.0$ equiv.) in $\mathrm{MeCN}(5.0 \mathrm{~mL})$ delivered 31 (41.0 mg, 70\%) after purification via silica gel column chromatography with $2.5 \%$ ethyl acetate/hexanes ( $R_{\mathrm{f}}=0.35,5 \%$ ethyl acetate/hexanes). 1 H NMR (400 MHz, $\left.\mathrm{CDCl}_{3}\right) \delta$ 7.40-7.27 (m, 5 H), 7.21 (t, $\left.J=7.6 \mathrm{~Hz}, 1 \mathrm{H}\right), 7.06$ (t, $\left.J=9.6 \mathrm{~Hz}, 2 \mathrm{H}\right), 5.12$ (s, $2 \mathrm{H}), 3.87$ (s, $2 \mathrm{H}), 2.48$ (s, $3 \mathrm{H}), 1.32$ (s, $12 \mathrm{H}) ; \mathbf{1 3 C}$ NMR (100 MHz, $\left.\mathrm{CDCl}_{3}\right) \delta$ 172.0, 143.5, 138.8, 136.2 129.6, 128.5, 128.4, 128.2, 128.0, 127.4, 83.7, 66.4, 41.5, 24.9, 22.6; HRMS-DART $(\mathrm{m} / \mathrm{z})$ : calculated for $\mathrm{C}_{22} \mathrm{H}_{27} \mathrm{O}_{4} \mathrm{~B}[\mathrm{M}]^{+} 366.1997$, found 366.2240 .
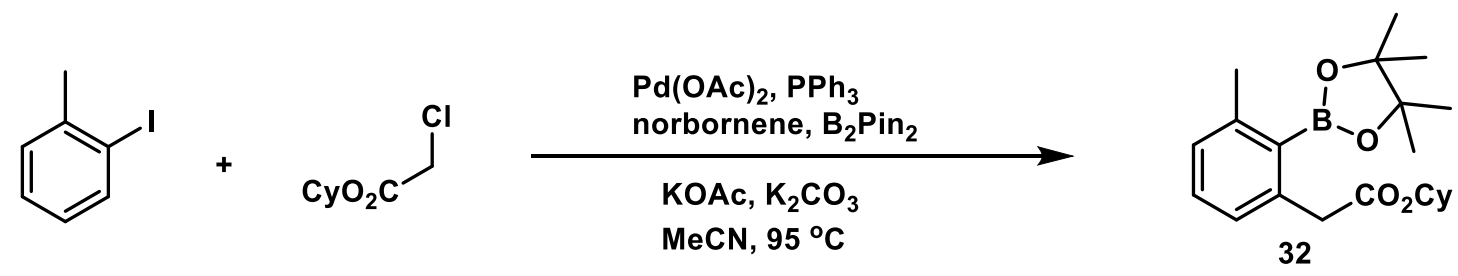

Compound 32 was synthesized following the General Procedure for the Alkylborylation 
of aryl iodides. The reaction of 2-iodotoluene (34.9 $\mathrm{mg}, 0.160 \mathrm{mmol}, 1.0$ equiv.), cyclohexyl chloroacetate (113 mg, $0.640 \mathrm{mmol}, 4.0$ equiv.), $\mathrm{Pd}(\mathrm{OAc})_{2}$ (2.5 mg, $0.0112 \mathrm{mmol}, 7 \%$ \%), $\mathrm{PPh}_{3}(5.9$ $\mathrm{mg}, 0.0224 \mathrm{mmol}, 14 \%$ ), norbornene (22.6 mg, $0.240 \mathrm{mmol}, 1.5$ equiv.), $\mathrm{K}_{2} \mathrm{CO}_{3}(66.2 \mathrm{mg}, 0.480$ mmol, 3.0 equiv.), KOAc (102 mg, $1.04 \mathrm{mmol}, 6.5$ equiv.) and $\mathrm{B}_{2} \mathrm{pin}_{2}(81.3 \mathrm{mg}, 0.320 \mathrm{mmol}, 2.0$ equiv.) in $\mathrm{MeCN}(5.0 \mathrm{~mL})$ delivered $32(39.0 \mathrm{mg}, 68 \%)$ after purification via silica gel column chromatography with $2.5 \%$ ethyl acetate/hexanes ( $R_{\mathrm{f}}=0.4,5 \%$ ethyl acetate/hexanes). 1 H NMR $\left(400 \mathrm{MHz}, \mathrm{CDCl}_{3}\right) \delta 7.19$ (t, $\left.J=7.6 \mathrm{~Hz}, 1 \mathrm{H}\right), 7.04(\mathrm{~d}, J=7.6 \mathrm{~Hz}, 2 \mathrm{H}), 4.80-4.66(\mathrm{~m}, 1 \mathrm{H}), 3.77$ (s, 2 H), 2.46 (s, 3 H), 1.88-1.74 (m, 2 H), 1.72-1.61 (m, 2 H), 1.55-1.14 (m, 18 H); 13C NMR $\left(125 \mathrm{MHz}, \mathrm{CDCl}_{3}\right) \delta 171.7,143.1,139.1,129.5,128.2,127.1,83.7,72.8,41.8,31.6,25.4,25.0$, 23.7, 22.6; HRMS-DART (m/z): calculated for $\mathrm{C}_{21} \mathrm{H}_{32} \mathrm{O}_{4} \mathrm{~B}[\mathrm{M}]^{+}$359.2388, found 359.2392.
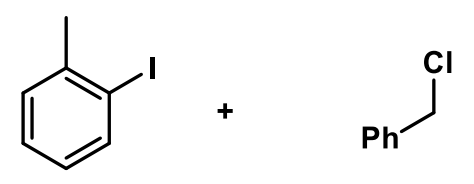

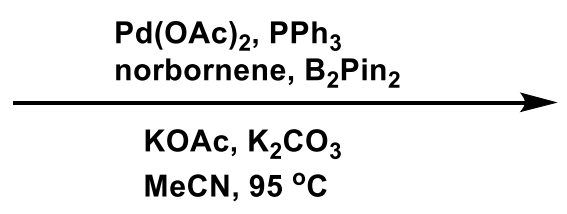

$\mathrm{MeCN}, 95^{\circ} \mathrm{C}$

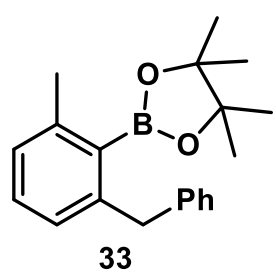

33

Compound 33 was synthesized following the General Procedure for the Alkylborylation

of aryl iodides. The reaction of 2-iodotoluene ( $34.9 \mathrm{mg}, 0.160 \mathrm{mmol}, 1.0$ equiv.), benzyl chloride (80.6 mg, 0.640 mmol, 4.0 equiv.), $\mathrm{Pd}(\mathrm{OAc})_{2}$ (2.5 mg, $0.0112 \mathrm{mmol}, 7$ \%), $\mathrm{PPh}_{3}$ (5.9 mg, 0.0224 mmol, $14 \%$ ), norbornene (22.6 mg, $0.240 \mathrm{mmol}, 1.5$ equiv.), $\mathrm{K}_{2} \mathrm{CO}_{3}(66.2 \mathrm{mg}, 0.480 \mathrm{mmol}, 3.0$ equiv.), KOAc (102 mg, $1.04 \mathrm{mmol}, 6.5$ equiv.) and $\mathrm{B}_{2} \operatorname{pin}_{2}(81.3 \mathrm{mg}, 0.320 \mathrm{mmol}, 2.0$ equiv.) in $\mathrm{MeCN}(5.0 \mathrm{~mL})$ delivered $33(26.6 \mathrm{mg}, 54 \%)$ after purification via silica gel column chromatography with $2.5 \%$ ethyl acetate/hexanes ( $R_{\mathrm{f}}=0.35,5 \%$ ethyl acetate/hexanes). 1 H NMR $\left(400 \mathrm{MHz}, \mathrm{CDCl}_{3}\right) \delta$ 7.30-7.22 (m, $\left.2 \mathrm{H}\right), 7.15$ (t, $\left.J=8.4 \mathrm{~Hz}, 4 \mathrm{H}\right), 7.00$ (d, $\left.J=7.6 \mathrm{~Hz}, 1 \mathrm{H}\right), 6.87$ (d, $J=7.6 \mathrm{~Hz}, 1 \mathrm{H}), 4.12$ (s, $2 \mathrm{H}), 2.44$ (s, $3 \mathrm{H}), 1.30$ (s, $12 \mathrm{H}) ; \mathbf{1 3 C}$ NMR $\left(100 \mathrm{MHz}, \mathrm{CDCl}_{3}\right) \delta$ 144.6, 142.0, 141.8, 129.4, 129.0, 128.2, 127.1, 126.7, 125.7, 83.7, 41.6, 25.0, 22.4; HRMSDART (m/z): calculated for $\mathrm{C}_{20} \mathrm{H}_{26} \mathrm{O}_{2} \mathrm{~B}[\mathrm{M}]^{+} 309.2020$, found 309.1892 . 

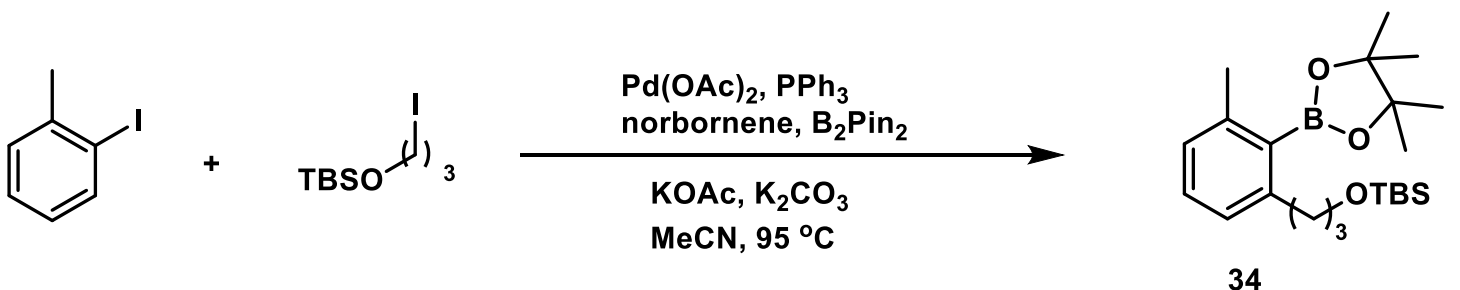

Compound 34 was synthesized following the General Procedure for the Alkylborylation of aryl iodides. The reaction of 2-iodotoluene (34.9 mg, $0.160 \mathrm{mmol}, 1.0$ equiv.), 1-(tertButyldimethylsilyloxy)-3-iodopropane (192 mg, $0.640 \mathrm{mmol}, 4.0$ equiv.), $\mathrm{Pd}(\mathrm{OAc})_{2}(2.5 \mathrm{mg}$, $0.0112 \mathrm{mmol}, 7$ \%), $\mathrm{PPh}_{3}$ (5.9 mg, $0.0224 \mathrm{mmol}, 14$ \%), norbornene (22.6 mg, $0.240 \mathrm{mmol}, 1.5$ equiv.), $\mathrm{K}_{2} \mathrm{CO}_{3}$ (66.2 mg, $0.480 \mathrm{mmol}, 3.0$ equiv.), KOAc (102 mg, $1.04 \mathrm{mmol}, 6.5$ equiv.) and $\mathrm{B}_{2} \operatorname{pin}_{2}(81.3 \mathrm{mg}, 0.320 \mathrm{mmol}, 2.0$ equiv.) in $\mathrm{MeCN}(5.0 \mathrm{~mL})$ delivered 34 (13.1 mg, 21\%) after purification via silica gel column chromatography with $2 \%$ ethyl acetate/hexanes $\left(\mathrm{R}_{\mathrm{f}}=0.45,5 \%\right.$ ethyl acetate/hexanes). 1H NMR $\left(400 \mathrm{MHz}^{\mathrm{CDCl}} \mathrm{CDC}_{3} \delta 7.15\right.$ (t, $\left.J=7.6 \mathrm{~Hz}, 1 \mathrm{H}\right), 6.96$ (t, $J=7.6$ Hz, 2 H), 3.64 (t, $J=6.8$ Hz, 2 H), 2.70 (t, $J=8.0$ Hz, 2 H), 2.39 (s, 3 H), 1.88-1.74 (m, 2 H), 1.38 (s, $12 \mathrm{H}), 0.90$ (s, $9 \mathrm{H}), 0.05$ (s, $6 \mathrm{H}) ; 13 \mathrm{C} \mathrm{NMR}\left(100 \mathrm{MHz}, \mathrm{CDCl}_{3}\right) \delta$ 146.3, 141.6, 129.1, 126.6, 125.8, 83.7, 62.9, 35.7, 32.8, 26.0, 25.0, 22.2, 18.4, -5.26.; HRMS-DART (m/z): calculated for $\mathrm{C}_{22} \mathrm{H}_{40} \mathrm{SiO}_{3} \mathrm{~B}[\mathrm{M}+\mathrm{H}]^{+}$391.2834, found 391.2838.
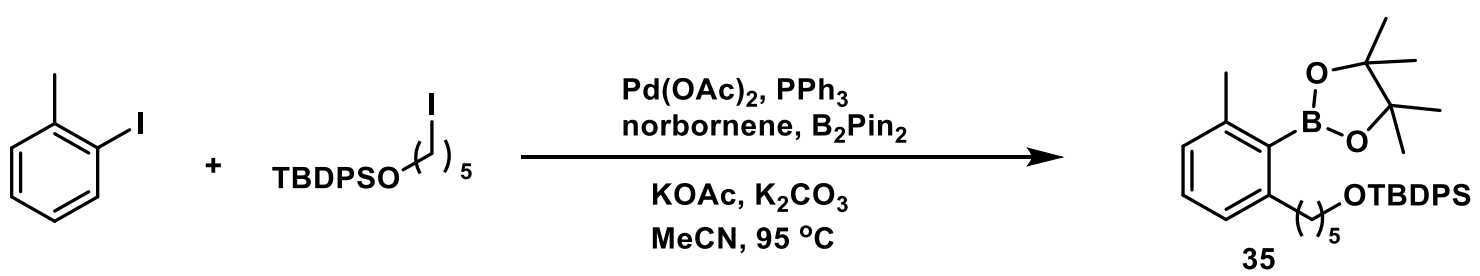

Compound 35 was synthesized following the General Procedure for the Alkylborylation of aryl iodides. The reaction of 2-iodotoluene (34.9 mg, $0.160 \mathrm{mmol}, 1.0$ equiv.), tert-Butyl(5iodo-pentyloxy)diphenylsilane (289 mg, $0.640 \mathrm{mmol}, 4.0$ equiv.), $\mathrm{Pd}(\mathrm{OAc})_{2}(2.5 \mathrm{mg}, 0.0112 \mathrm{mmol}$, $7 \%$ ), $\mathrm{PPh}_{3}\left(5.9 \mathrm{mg}, 0.0224 \mathrm{mmol}, 14 \%\right.$ ), norbornene (22.6 mg, $0.240 \mathrm{mmol}, 1.5$ equiv.), $\mathrm{K}_{2} \mathrm{CO}_{3}$ (66.2 mg, 0.480 mmol, 3.0 equiv.), KOAc (102 mg, 1.04 mmol, 6.5 equiv.) and $B_{2}$ pin $_{2}$ (81.3 mg, $0.320 \mathrm{mmol}, 2.0$ equiv.) in $\mathrm{MeCN}(5.0 \mathrm{~mL})$ delivered 35 (20.8 mg, 24\%) after purification via silica gel column chromatography with $3 \%$ ethyl acetate/hexanes $\left(R_{f}=0.3\right.$, 5\% ethyl 
acetate/hexanes). 1H NMR (400 MHz, $\left.\mathrm{CDCl}_{3}\right) \delta 7.66(\mathrm{dd}, J=1.2,7.6 \mathrm{~Hz}, 4 \mathrm{H}), 7.45-7.32(\mathrm{~m}, 6$ H), $7.14(\mathrm{t}, J=7.6 \mathrm{~Hz}, 1 \mathrm{H}), 6.94(\mathrm{t}, J=7.2 \mathrm{~Hz}, 2 \mathrm{H}), 3.66$ (t, $J=6.4 \mathrm{~Hz}, 2 \mathrm{H}), 2.63(\mathrm{t}, J=8.0 \mathrm{~Hz}$, $2 \mathrm{H}), 2.40$ (s, $3 \mathrm{H}), 1.64-1.50$ (m, 4 H), 1.45-1.33 (m, 14 H), 1.04 (s, 9 H); 13C NMR (100 MHz, $\left.\mathrm{CDCl}_{3}\right) \delta 146.8,141.6,135.6,134.2,129.5,129.1,127.6,126.6,125.7,83.6,64.0,36.6,32.7,32.6$, 26.9, 26.0, 25.0, 22.2, 19.2; HRMS-DART (m/z): calculated for $\mathrm{C}_{34} \mathrm{H}_{48} \mathrm{SiO}_{3} \mathrm{~B}[\mathrm{M}+\mathrm{H}]^{+}$543.3460, found 543.3468 .
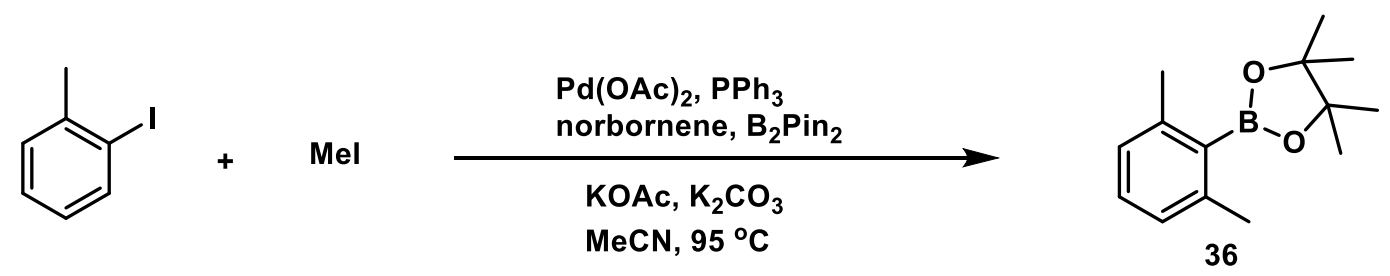

Compound $\mathbf{3 6}$ was synthesized following the General Procedure for the Alkylborylation of aryl iodides (MeI was 7.0 equiv.). The reaction of 2-iodotoluene (34.9 mg, $0.160 \mathrm{mmol}, 1.0$ equiv.), methyl iodide (159 mg, $1.12 \mathrm{mmol}, 7.0$ equiv.), $\mathrm{Pd}(\mathrm{OAc})_{2}(2.5 \mathrm{mg}, 0.0112 \mathrm{mmol}, 7 \%$ ), $\mathrm{PPh}_{3}$ (5.9 mg, $0.0224 \mathrm{mmol}, 14 \%$ ), norbornene (22.6 mg, $0.240 \mathrm{mmol}, 1.5$ equiv.), $\mathrm{K}_{2} \mathrm{CO}_{3}$ (66.2 $\mathrm{mg}, 0.480 \mathrm{mmol}, 3.0$ equiv.), KOAc (102 mg, $1.04 \mathrm{mmol}, 6.5$ equiv.) and $\mathrm{B}_{2} \mathrm{pin}_{2}(81.3 \mathrm{mg}, 0.320$ mmol, 2.0 equiv.) in $\mathrm{MeCN}(5.0 \mathrm{~mL})$ delivered $36(22.3 \mathrm{mg}, 60 \%)$ after purification via silica gel column chromatography with $2 \%$ ethyl acetate/hexanes $\left(\mathrm{R}_{\mathrm{f}}=0.25,5 \%\right.$ ethyl acetate/hexanes). $1 \mathrm{H}$ NMR (400 MHz, CDCl $) \delta 7.12(\mathrm{t}, J=7.6 \mathrm{~Hz}, 1 \mathrm{H}), 6.94$ (d, $J=7.6 \mathrm{~Hz}, 2 \mathrm{H}), 2.40$ (s, $6 \mathrm{H}), 1.39$ (s, $12 \mathrm{H})$.
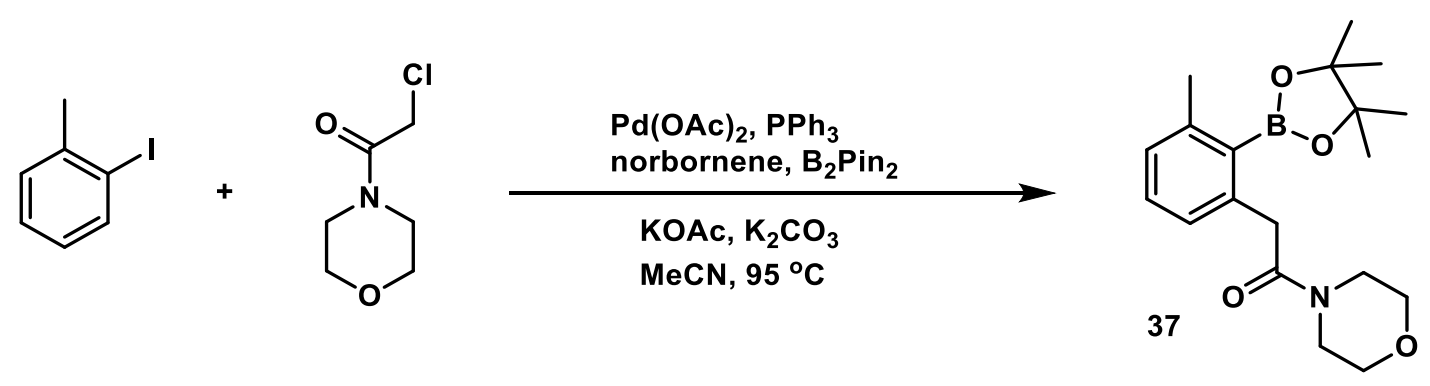

Compound 37 was synthesized following the General Procedure for the Alkylborylation of aryl iodides. The reaction of 2-iodotoluene (34.9 mg, $0.160 \mathrm{mmol}, 1.0$ equiv.), $\mathrm{N}$ (Chloroacetyl)morpholine (104 mg, $0.640 \mathrm{mmol}, 4.0$ equiv.), $\mathrm{Pd}(\mathrm{OAc})_{2}(2.5 \mathrm{mg}, 0.0112 \mathrm{mmol}$, 
$7 \%$ ), $\mathrm{PPh}_{3}$ (5.9 mg, $0.0224 \mathrm{mmol}, 14 \%$ ), norbornene (22.6 mg, $0.240 \mathrm{mmol}, 1.5$ equiv.), $\mathrm{K}_{2} \mathrm{CO}_{3}$ (66.2 mg, $0.480 \mathrm{mmol}, 3.0$ equiv.), KOAc (102 mg, $1.04 \mathrm{mmol}, 6.5$ equiv.) and $\mathrm{B}_{2} \mathrm{pin}_{2}(81.3 \mathrm{mg}$, $0.320 \mathrm{mmol}, 2.0$ equiv.) in $\mathrm{MeCN}(5.0 \mathrm{~mL})$ delivered $37(33.7 \mathrm{mg}, 61 \%)$ after purification via silica gel column chromatography with $10 \%$ ethyl acetate/hexanes $\left(\mathrm{R}_{\mathrm{f}}=0.25,10 \%\right.$ ethyl acetate/hexanes). 1H NMR (400 MHz, $\left.\mathrm{CDCl}_{3}\right) \delta 7.20(\mathrm{t}, J=7.6 \mathrm{~Hz}, 1 \mathrm{H}), 7.03(\mathrm{~d}, J=7.6 \mathrm{~Hz}, 1$ H), 6.99 (d, $J=7.6 \mathrm{~Hz}, 1 \mathrm{H}), 3.87$ (s, $2 \mathrm{H}), 3.64$ (s, $4 \mathrm{H}), 3.51$ (t, $J=4.0 \mathrm{~Hz}, 2 \mathrm{H}), 3.44$ (t, $J=5.2$ $\mathrm{Hz}, 2 \mathrm{H}), 2.44$ (s, $3 \mathrm{H}), 1.36$ (s, $12 \mathrm{H})$; 13C NMR (100 MHz, $\left.\mathrm{CDCl}_{3}\right) \delta$ 170.4, 143.2, 139.3, 129.7, 128.0, 125.4, 83.7, 66.8, 66.6, 46.2, 42.1, 41.0, 25.0, 22.6; HRMS-DART (m/z): calculated for $\mathrm{C}_{19} \mathrm{H}_{29} \mathrm{NO}_{4} \mathrm{~B}[\mathrm{M}+\mathrm{H}]^{+} 346.2184$, found 346.2192 .
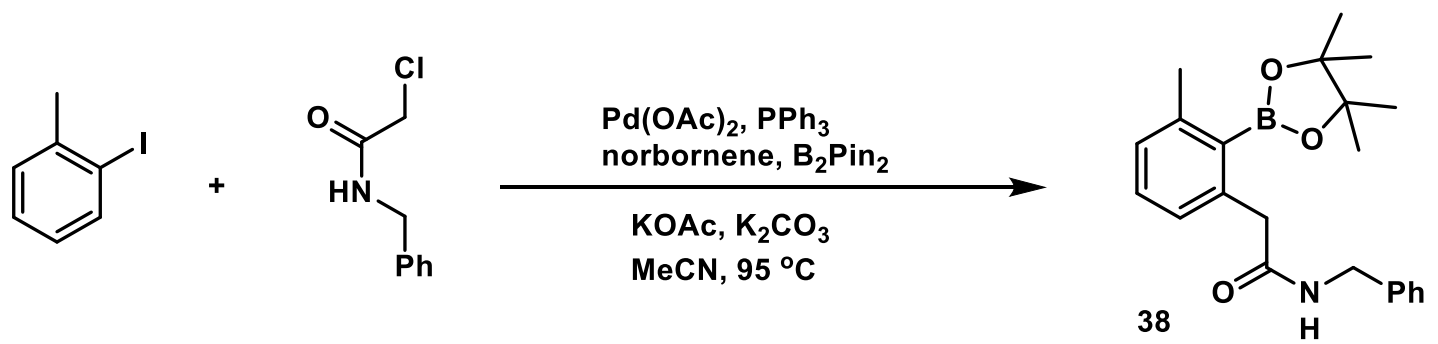

Compound 38 was synthesized following the General Procedure for the Alkylborylation of aryl iodides. The reaction of 2-iodotoluene (34.9 mg, $0.160 \mathrm{mmol}, 1.0$ equiv.), Nbenzylchloroacetamide (117 mg, $0.640 \mathrm{mmol}, 4.0$ equiv.), $\mathrm{Pd}(\mathrm{OAc})_{2}(2.5 \mathrm{mg}, 0.0112 \mathrm{mmol}, 7 \%$ ), $\mathrm{PPh}_{3}$ (5.9 mg, $0.0224 \mathrm{mmol}, 14 \%$ ), norbornene (22.6 mg, 0.240 mmol, 1.5 equiv.), $\mathrm{K}_{2} \mathrm{CO}_{3}$ (66.2 $\mathrm{mg}, 0.480 \mathrm{mmol}, 3.0$ equiv.), KOAc (102 mg, $1.04 \mathrm{mmol}, 6.5$ equiv.) and $\mathrm{B}_{2} \mathrm{pin}_{2}(81.3 \mathrm{mg}, 0.320$ mmol, 2.0 equiv.) in $\mathrm{MeCN}(5.0 \mathrm{~mL})$ delivered 38 (33.3 mg, 57\%) after purification via silica gel column chromatography with $10 \%$ ethyl acetate/hexanes ( $R_{\mathrm{f}}=0.35,15 \%$ ethyl acetate/hexanes). 1H NMR (400 MHz, $\left.\mathrm{CDCl}_{3}\right) \delta$ 7.33-7.17 (m, $\left.7 \mathrm{H}\right), 7.08$ (d, $\left.J=7.6 \mathrm{~Hz}, 1 \mathrm{H}\right), 6.64(\mathrm{br}, 1 \mathrm{H}), 4.38$ (d, $J=5.6 \mathrm{~Hz}, 2 \mathrm{H}), 3.68$ (s, $2 \mathrm{H}), 2.46(\mathrm{~s}, 3 \mathrm{H}), 1.31(\mathrm{~s}, 12 \mathrm{H}) ; \mathbf{1 3 C} \mathbf{N M R}\left(100 \mathrm{MHz}, \mathrm{CDCl}_{3}\right) \delta$ 171.4, 143.3, 139.8, 138.5, 130.2, 128.5, 128.4, 127.7, 127.2, 127.1, 84.3, 44.5, 43.6, 24.8, 22.5; HRMS-DART (m/z): calculated for $\mathrm{C}_{22} \mathrm{H}_{29} \mathrm{NO}_{3} \mathrm{~B}[\mathrm{M}+\mathrm{H}]^{+} 366.2235$, found 366.2240 .

\section{Diversification of the Alkyaborylation products}




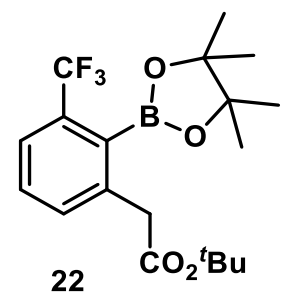

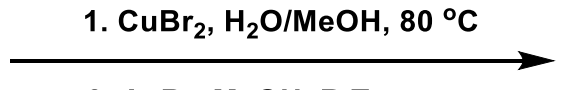

2. $\mathrm{AcBr}, \mathrm{MeOH}$, R.T.

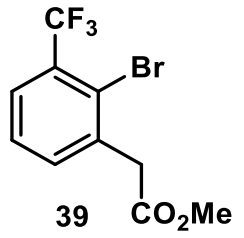

Bromide 39: The boronic ester $(100 \mathrm{mg}, 0.259 \mathrm{mmol})$ was dissolved in $\mathrm{MeOH}(2.6 \mathrm{~mL})$ and water $(2.6 \mathrm{~mL})$ and stirring was initiated ${ }^{8} . \mathrm{CuBr}_{2}(173 \mathrm{mg}, 0.777 \mathrm{mmol})$ was added and the reaction mixture was heated to $80^{\circ} \mathrm{C}$. After the reaction was complete, as judged by TLC, methanol was removed under reduced pressure. The remaining material was extracted with EtOAc $(3 \times 5$ $\mathrm{mL})$. The combined organic fractions were washed with brine $(1 \times 4 \mathrm{~mL})$, dried over $\mathrm{Na}_{2} \mathrm{SO}_{4}$, filtered and concentrated under reduced pressure. Then, the crude material was dropwise added into an ice-cold solution of acetyl bromide $(0.073 \mathrm{~mL}, 0.988 \mathrm{mmol})$ in $\mathrm{MeOH}(2.0 \mathrm{~mL})$. Then, the reaction mixture was stirred at room temperature for $12 \mathrm{~h}$. After the reaction was complete, as judged by TLC, it was extracted with EtOAc $(3 \times 5 \mathrm{~mL})$. The combined organic fractions were washed with brine $(1 \times 4 \mathrm{~mL})$, dried over $\mathrm{Na}_{2} \mathrm{SO}_{4}$, filtered and concentrated under reduced pressure The crude material was purified by preparative TLC (silica gel, 10\% ethyl acetate in hexanes) to afford 39 (30.0 mg, 26\%) ( $\mathrm{R}_{\mathrm{f}}=0.2,10 \%$ ethyl acetate/hexanes). $1 \mathbf{H ~ N M R}\left(500 \mathrm{MHz}, \mathrm{CDCl}_{3}\right) \delta$ $7.66(\mathrm{dd}, J=7.8,1.5 \mathrm{~Hz}, 1 \mathrm{H}), 7.50(\mathrm{dd}, J=7.6,1.3 \mathrm{~Hz}, 1 \mathrm{H}), 7.42$ (t, $J=7.7 \mathrm{~Hz}, 1 \mathrm{H}), 3.93(\mathrm{~s}, 2 \mathrm{H})$, 3.76 (s, 2H); 13C NMR (176 MHz, $\left.\mathrm{CDCl}_{3}\right) \delta 170.4,137.0,134.8,131.0$ (q, $\left.J=31.7 \mathrm{~Hz}\right), 127.3$, 126.9 (q, $J=7.0 \mathrm{~Hz}), 123.0$ (q, $J=272.8 \mathrm{~Hz}), 122.8,52.4,42.0 ;$ 19F NMR $(376 \mathrm{MHz}, \mathrm{CDCl} 3) \delta$ -133.1; HRMS-DART (m/z): calculated for $\mathrm{C}_{10} \mathrm{H}_{8} \mathrm{BrF}_{3} \mathrm{O}_{2}[\mathrm{M}+\mathrm{H}]^{+} 296.9733$ and $[\mathrm{M}+\mathrm{H}+2]^{+}$ 298.9712, found 296.9733 and 298.9708.

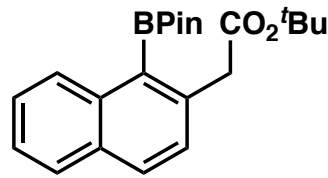

26

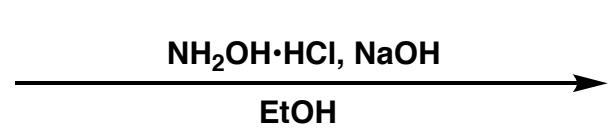

EtOH

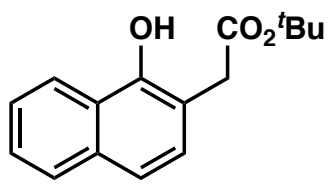

41

Naphthol 41: To a solution of the boronic ester $(75.0 \mathrm{mg}, 0.204 \mathrm{mmol})$ in EtOH $(2.0 \mathrm{~mL})$, 
$\mathrm{NH}_{2} \mathrm{OH} \cdot \mathrm{HCl}(21.2 \mathrm{mg}, 0.305 \mathrm{~mol})$ and $\mathrm{NaOH}(16.3 \mathrm{mg}, 0.407 \mathrm{mmol})$ were added and the mixture was stirred at room temperature for $12 \mathrm{~h}^{9}$. The reaction progress was monitored by TLC. Work-up of the reaction involved evaporating the solvent under reduced pressure, then extraction with dichloromethane. The solvent was removed under vacuum and the product was purified by column chromatography (silica gel, 0, 5, 10\% ethyl acetate in hexanes as eluent) to give $\mathbf{4 1}$ (34.0 $\mathrm{mg}, 65 \%$ ) $\left(\mathrm{R}_{\mathrm{f}}=0.45,10 \%\right.$ ethyl acetate/hexanes). $1 \mathbf{H}$ NMR $\left(600 \mathrm{MHz}, \mathrm{CDCl}_{3}\right) \delta 8.87(\mathrm{br}, 1 \mathrm{H}), 8.45-8.23$ (m, 1H), $7.81-7.73(\mathrm{~m}, 1 \mathrm{H}), 7.59-7.42(\mathrm{~m}, 2 \mathrm{H}), 7.37(\mathrm{~d}, J=8.3 \mathrm{~Hz}, 1 \mathrm{H}), 7.17(\mathrm{~d}, J=8.3 \mathrm{~Hz}$, 1H), 3.74 (s, 2H), 1.48 (s, 9H); 13C NMR (100 MHz, $\left.\mathrm{CDCl}_{3}\right) \delta$ 174.3, 151.7, 134.4, 128.7, 127.4, 126.3, 126.3, 125.4, 122.6, 120.2, 114.0, 83.4, 40.2, 28.1; HRMS-DART (m/z): calculated for $\mathrm{C}_{16} \mathrm{H}_{18} \mathrm{O}_{3}[\mathrm{M}]^{+}$258.1256, found 258.1265.
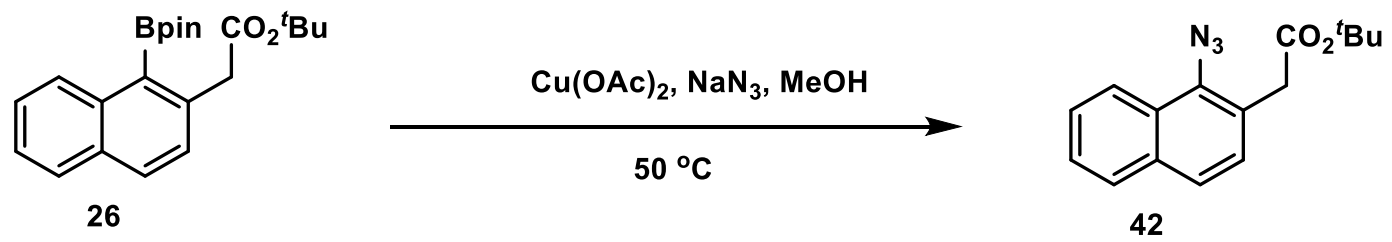

Azide 42: The boronic ester ${ }^{10}(58.9 \mathrm{mg}, 0.160 \mathrm{mmol})$ was dissolved in $\mathrm{MeOH}(2.7 \mathrm{~mL})$ and copper (II) acetate $(3.7 \mathrm{mg}, 0.0204 \mathrm{mmol})$ and sodium azide $(0.020 \mathrm{~mL}, 0.305 \mathrm{mmol})$ were added and the reaction mixture was heated to $50^{\circ} \mathrm{C}$ open to the air. After the reaction was complete, as judged by TLC, methanol was removed under reduced pressure. The residue was dissolved in EtOAc and washed with water $(1 \times 15 \mathrm{~mL})$. The organic layer was washed with brine $(1 \times 10 \mathrm{~mL})$, dried over $\mathrm{Na}_{2} \mathrm{SO}_{4}$, filtered and concentrated under reduced pressure. The crude material was purified by silica gel chromatography to give $42(43.0 \mathrm{mg}, 92 \%)\left(\mathrm{R}_{\mathrm{f}}=0.65,10 \%\right.$ ethyl acetate/hexanes). 1H NMR $\left(600 \mathrm{MHz}, \mathrm{CDCl}_{3}\right) \delta 8.16(\mathrm{~d}, J=8.5 \mathrm{~Hz}, 1 \mathrm{H}), 7.85(\mathrm{~d}, J=8.1 \mathrm{~Hz}, 1 \mathrm{H})$, $7.70(\mathrm{~d}, J=8.4 \mathrm{~Hz}, 1 \mathrm{H}), 7.58$ (ddd, $J=8.3,6.9,1.3 \mathrm{~Hz}, 1 \mathrm{H}), 7.52$ (ddd, $J=8.0,6.9,1.1 \mathrm{~Hz}, 1 \mathrm{H})$, $7.35(\mathrm{~d}, J=8.4 \mathrm{~Hz}, 1 \mathrm{H}), 3.84$ (s, 2H), $1.46(\mathrm{~s}, 9 \mathrm{H}) ; \mathbf{1 3 C} \mathbf{~ N M R}\left(100 \mathrm{MHz}, \mathrm{CDCl}_{3}\right) \delta 170.3,134.2$, 133.7, 128.4, 128.4, 126.9, 126.5, 126.3, 126.1, 122.3, 81.4, 39.1, 28.1; HRMS-DART (m/z): calculated for $\mathrm{C}_{16} \mathrm{H}_{17} \mathrm{~N}_{3} \mathrm{O}_{2}[\mathrm{M}]^{+}$283.1321, found 283.1326. 


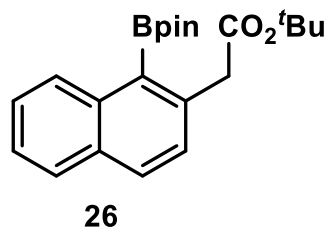

26

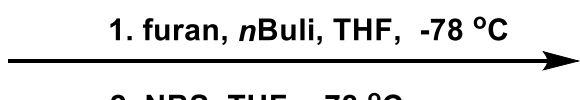

2. NBS, THF, $-78^{\circ} \mathrm{C}$

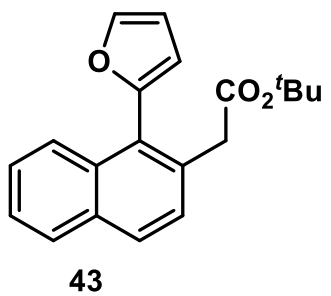

Furan 43: A solution of furan $(0.047 \mathrm{~mL}, 0.652 \mathrm{mmol})$ in THF $(2.2 \mathrm{~mL})$ was cooled to $78{ }^{\circ} \mathrm{C}$ and treated with BuLi $(0.291 \mathrm{~mL}, 0.652 \mathrm{mmol})^{11}$. The cooling bath was removed and the mixture was stirred at room temperature for $2 \mathrm{~h}$. The mixture was cooled to $-78^{\circ} \mathrm{C}$ and the boronic ester $(80.0 \mathrm{mg}, 0.217 \mathrm{mmol})$ was added dropwise as a solution in THF $(0.434 \mathrm{~mL})$. The mixture was stirred at $-78{ }^{\circ} \mathrm{C}$ for $6 \mathrm{~h}$. Then, a solution of NBS (46.4 mg, $\left.0.261 \mathrm{mmol}\right)$ in THF (0.87 mL) was added dropwise. After stirred at $-78^{\circ} \mathrm{C}$ for $1 \mathrm{~h}$ and at room temperature for $12 \mathrm{~h}, \mathrm{Na}_{2} \mathrm{~S}_{2} \mathrm{O}_{3}$ sat. was added. The reaction mixture was diluted with $\mathrm{Et}_{2} \mathrm{O}$ and water. The layers were separated and the aqueous layer was extracted with $\mathrm{Et}_{2} \mathrm{O}$. The combined organic layers were dried $\left(\mathrm{MgSO}_{4}\right)$, filtered and concentrated under vacuum. The crude material was purified by preparative TLC (silica gel, 10\% ethyl acetate in hexanes) to afford $30.0 \mathrm{mg}$ of $\mathbf{4 3}$ as a yellow oil in $45 \%$ yield $\left(\mathrm{R}_{\mathrm{f}}\right.$ $=0.6,10 \%$ ethyl acetate/hexanes). 1 H NMR (400 MHz, $\left.\mathrm{CDCl}_{3}\right) \delta 7.87(\mathrm{~d}, J=8.4 \mathrm{~Hz}, 1 \mathrm{H}), 7.84$ $(\mathrm{dd}, J=7.1,2.4 \mathrm{~Hz}, 1 \mathrm{H}), 7.71-7.66(\mathrm{~m}, 1 \mathrm{H}), 7.63(\mathrm{dd}, J=1.9,0.8 \mathrm{~Hz}, 1 \mathrm{H}), 7.51-7.40(\mathrm{~m}, 3 \mathrm{H})$, $6.60(\mathrm{dd}, J=3.2,1.9 \mathrm{~Hz}, 1 \mathrm{H}), 6.54(\mathrm{dd}, J=3.2,0.8 \mathrm{~Hz}, 1 \mathrm{H}), 3.62(\mathrm{~s}, 2 \mathrm{H}), 1.41(\mathrm{~s}, 9 \mathrm{H})$; 13C NMR $\left(150 \mathrm{MHz}, \mathrm{CDCl}_{3}\right) \delta 170.8,150.5,142.5,133.4,133.2,132.6,129.3,128.4,128.0,127.9,126.5$, 126.1 $125.7,111.3,110.7,80.8,41.1,28.0$; HRMS-DART $(\mathrm{m} / \mathrm{z})$ : calculated for $\mathrm{C}_{20} \mathrm{H}_{20} \mathrm{O}_{3}[\mathrm{M}]^{+}$ 308.1412, found 308.1413 .
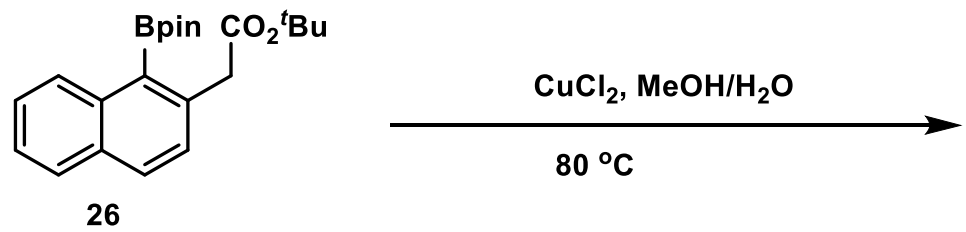

Chloride 44: The boronic ester was dissolved in $\mathrm{MeOH}(2.0 \mathrm{~mL})$, then Water $(2.0 \mathrm{~mL})$ and copper chloride $(82.1 \mathrm{mg}, 0.611 \mathrm{mmol})$ were added and the reaction mixture was heated to $80{ }^{\circ} \mathrm{C}$ open to the $\operatorname{air}^{10}$. After the reaction was complete, as judged by TLC, methanol was removed 
under reduced pressure. The residue was dissolved in EtOAc and washed with water $(1 \times 15 \mathrm{~mL})$. The organic layer was washed with brine $(1 \times 10 \mathrm{~mL})$, dried over $\mathrm{Na}_{2} \mathrm{SO}_{4}$, filtered and concentrated under reduced pressure. The crude material was purified by silica gel chromatography to give $\mathbf{4 4}$ in $96 \%$ yield $\left(\mathrm{R}_{\mathrm{f}}=0.6,10 \%\right.$ ethyl acetate/hexanes). $1 \mathrm{H} \mathrm{NMR}\left(600 \mathrm{MHz}, \mathrm{CDCl}_{3}\right) \delta 8.32(\mathrm{~d}, J=$ $8.5 \mathrm{~Hz}, 1 \mathrm{H}), 7.83(\mathrm{~d}, J=8.1 \mathrm{~Hz}, 1 \mathrm{H}), 7.74(\mathrm{~d}, J=8.4 \mathrm{~Hz}, 1 \mathrm{H}), 7.59$ (ddd, $J=8.3,6.9,1.2 \mathrm{~Hz}, 1 \mathrm{H}$ ), 7.52 (td, $J=7.5,6.9,1.0 \mathrm{~Hz}, 1 \mathrm{H}), 7.40(\mathrm{~d}, J=8.4 \mathrm{~Hz}, 1 \mathrm{H}), 3.92(\mathrm{~s}, 2 \mathrm{H}), 1.48(\mathrm{~s}, 9 \mathrm{H}) ; \mathbf{1 3 C}$ NMR $\left(150 \mathrm{MHz}, \mathrm{CDCl}_{3}\right) \delta 170.0,133.7,131.7,131.2,130.7,128.5,128.1,127.2,126.9,126.4,124.7$, 81.3, 41.3, 28.1; HRMS-DART (m/z): calculated for $\mathrm{C}_{16} \mathrm{H}_{17} \mathrm{ClO}_{2}[\mathrm{M}]^{+}$276.0917, found 276.0919. 


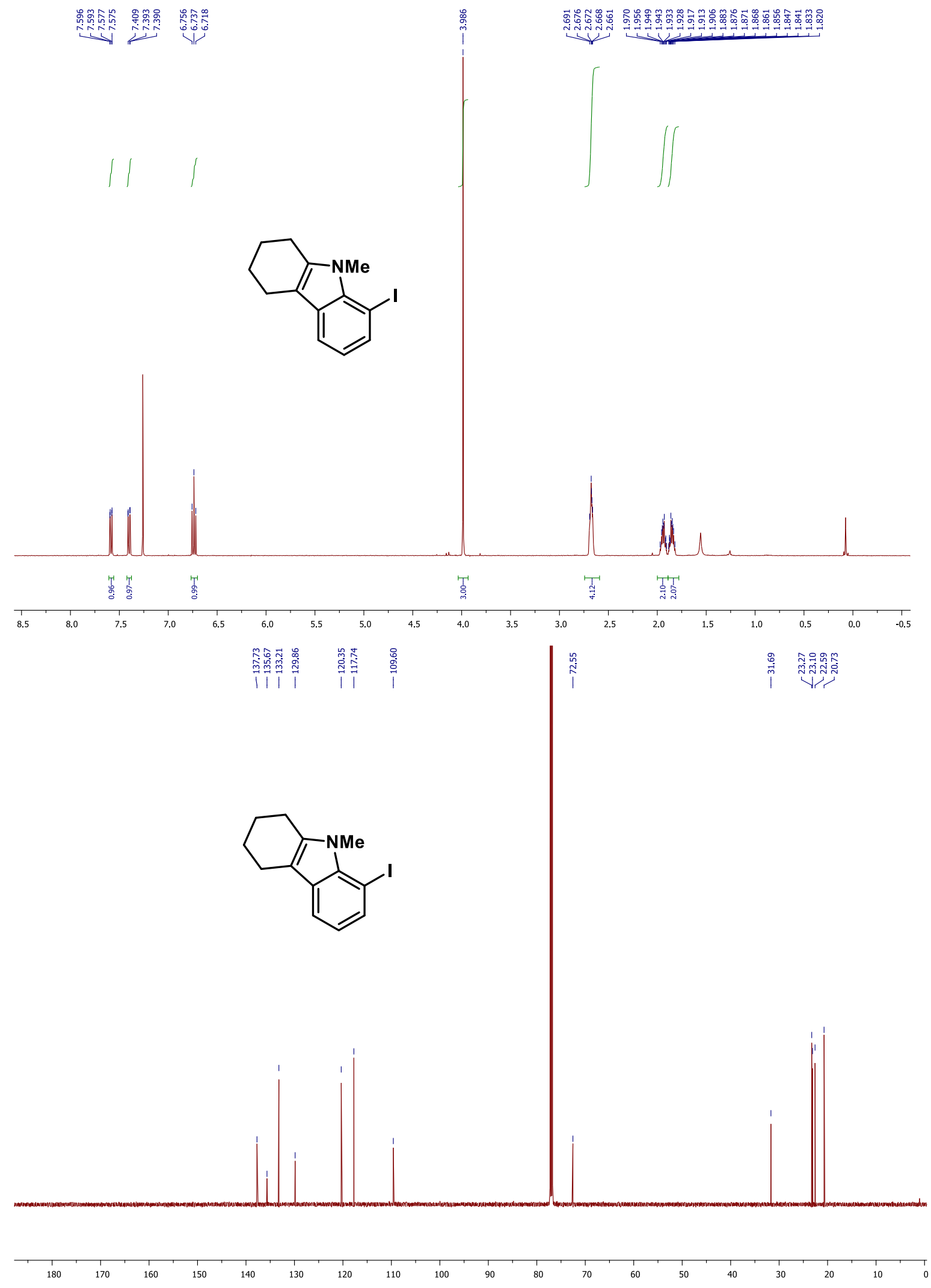




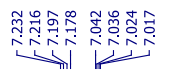

, 1 i̊n.

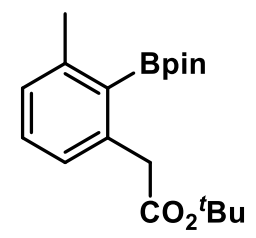

12

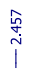

i vo
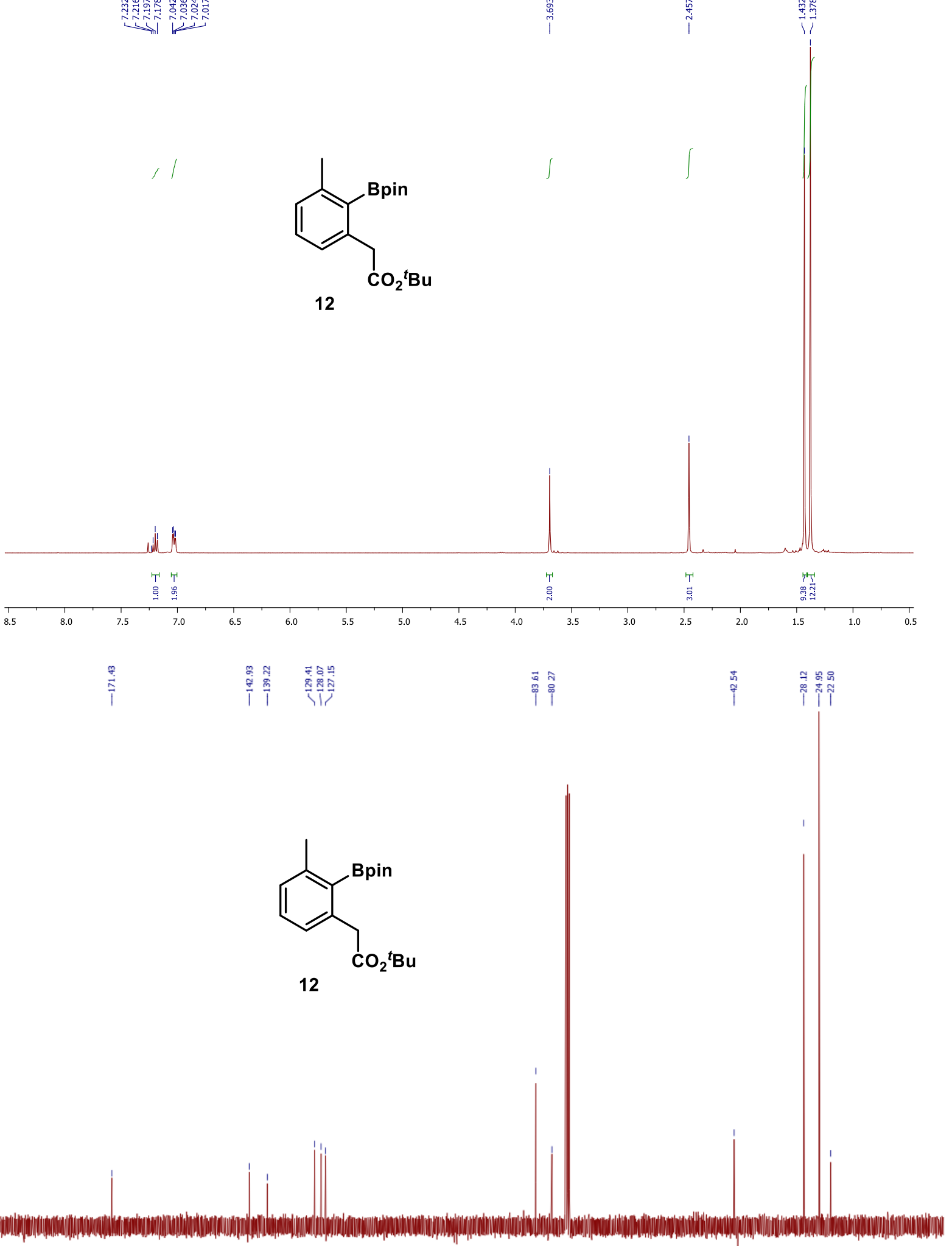

12

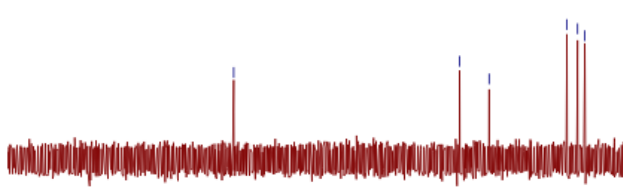

\begin{tabular}{ll}
$\vec{a}$ & \multirow{2}{*}{} \\
0 & 0 \\
0 & 0
\end{tabular}

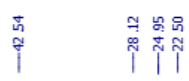

0.5

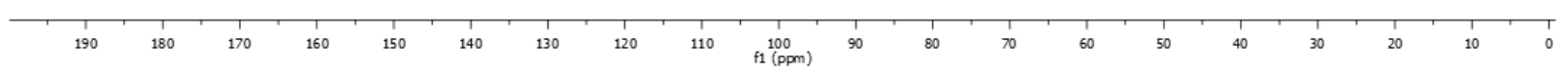

S29 


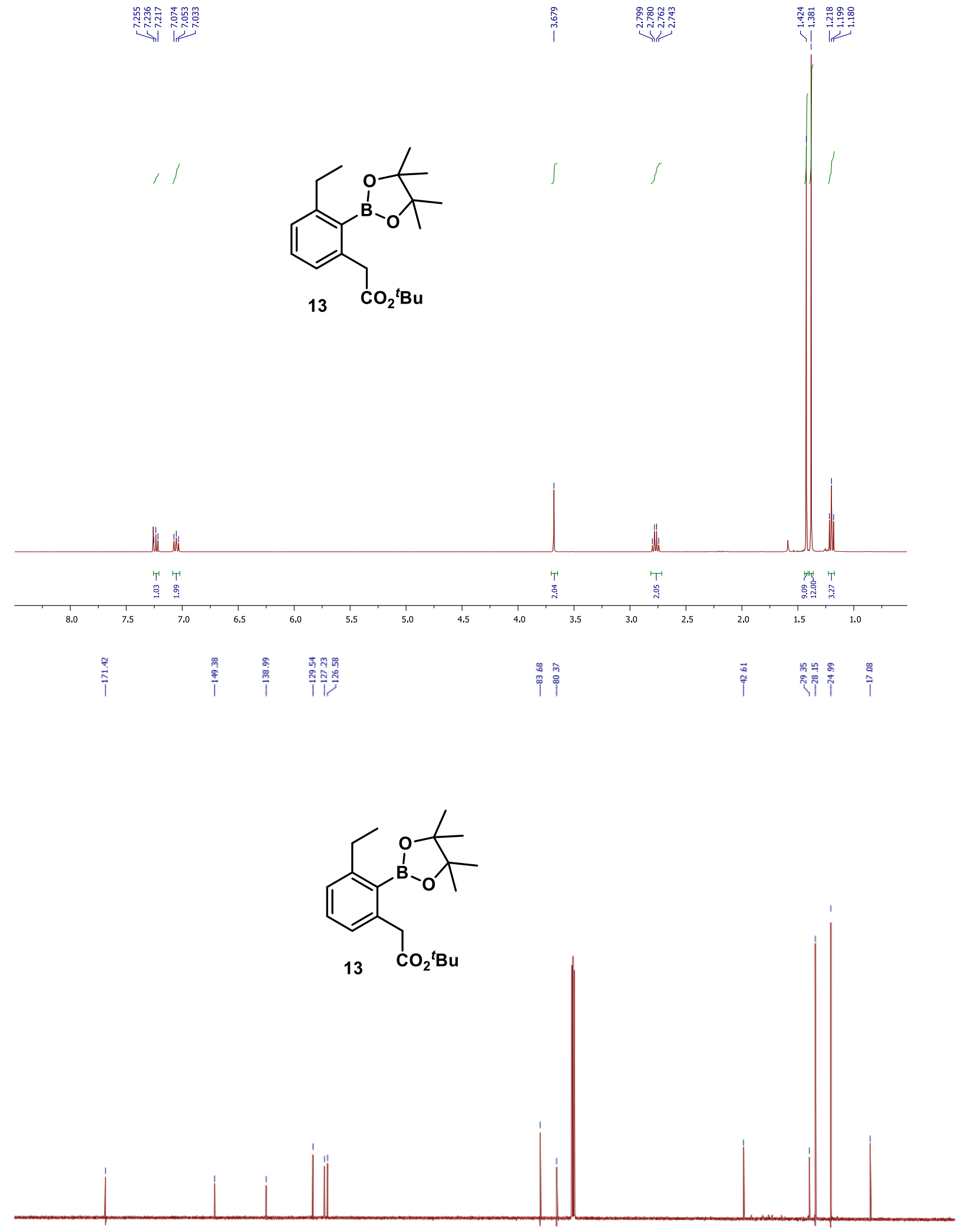




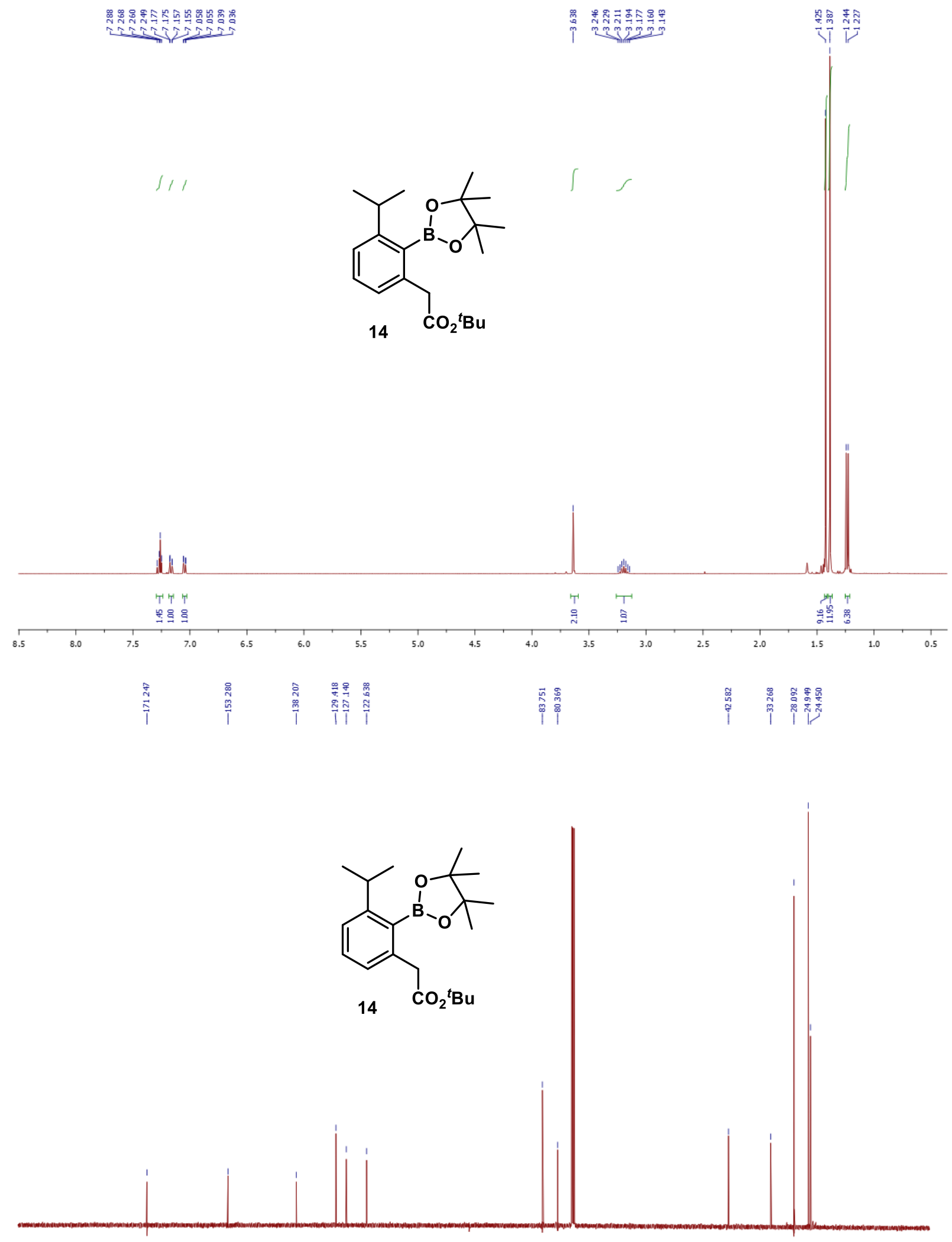



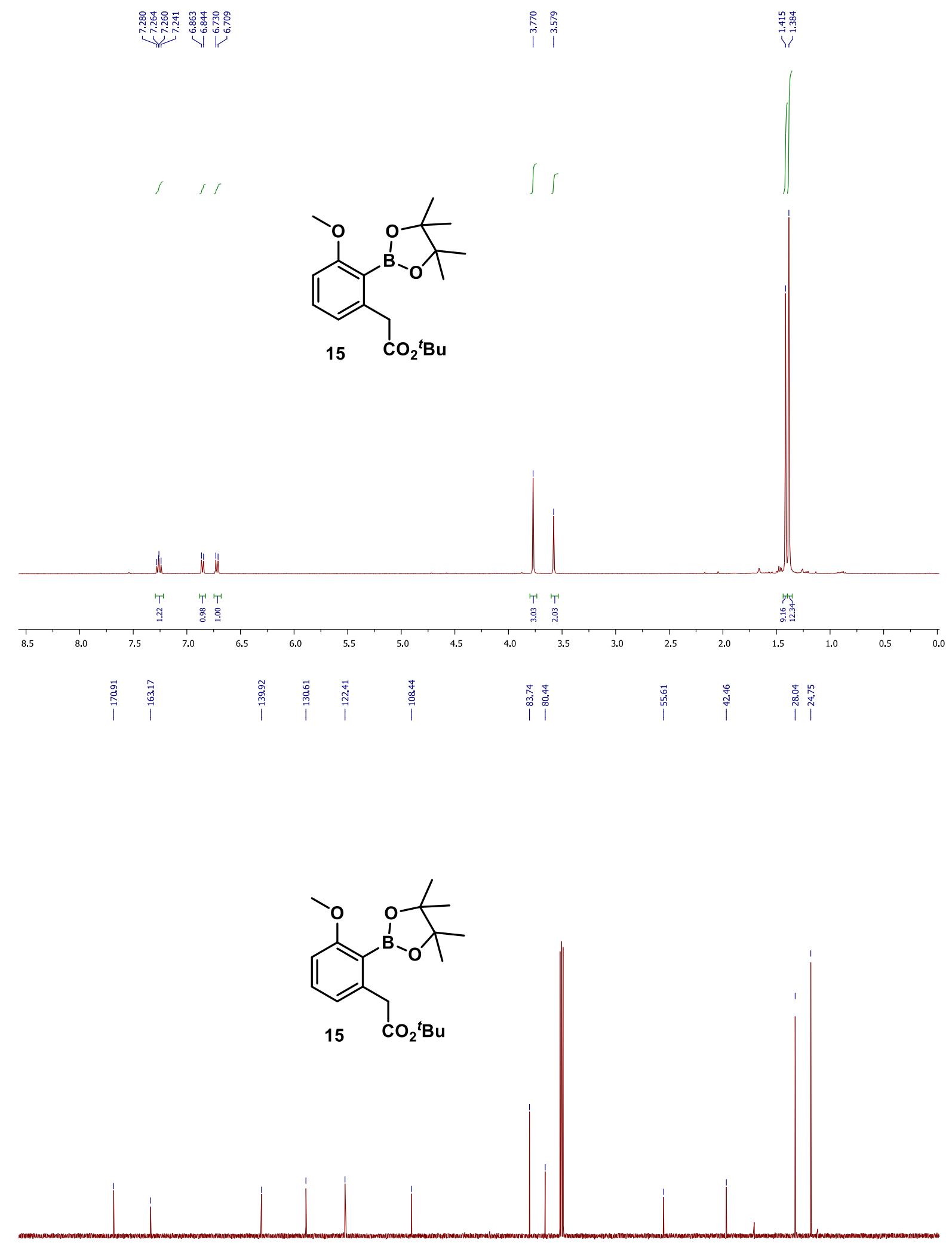

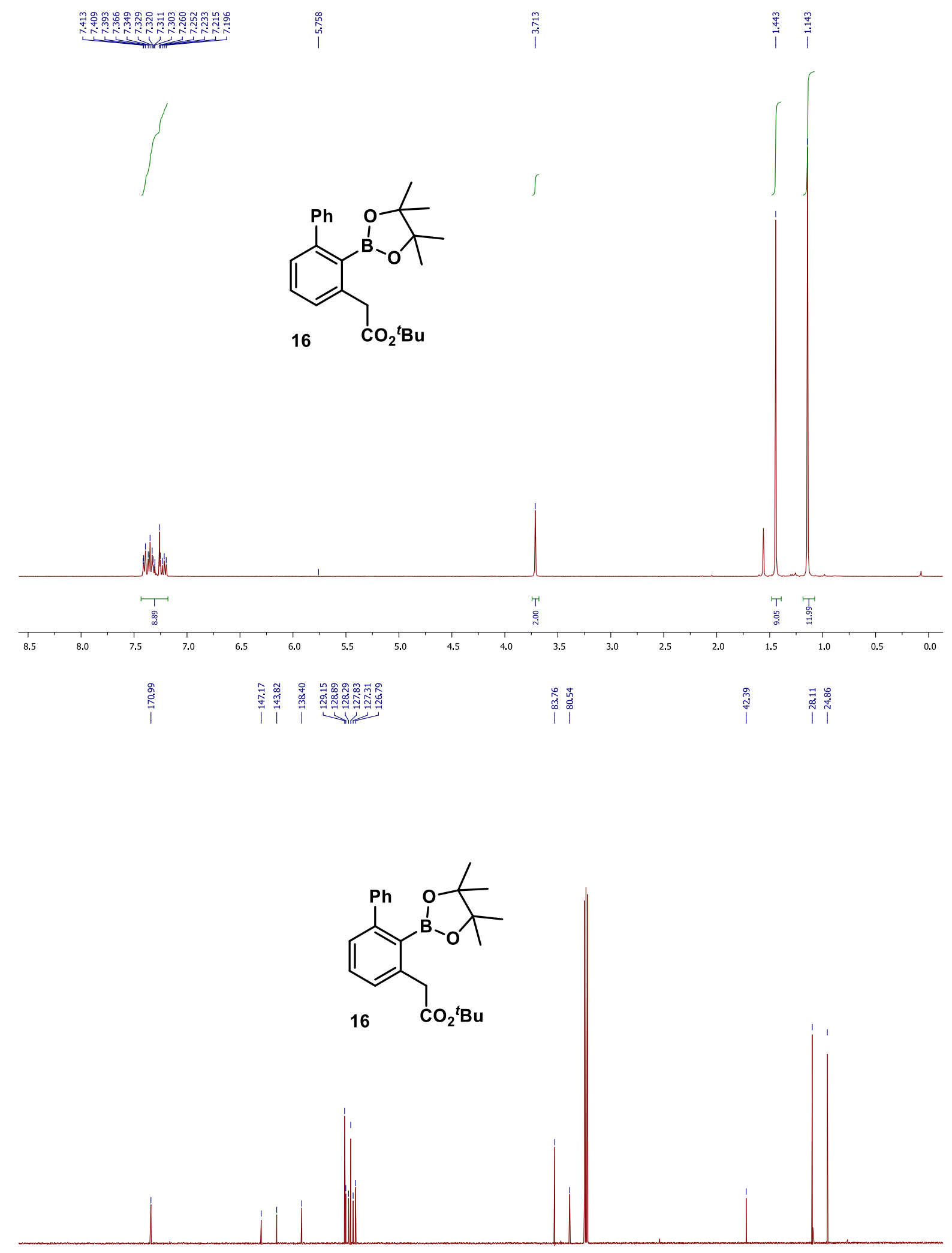


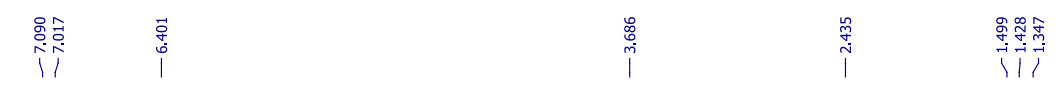
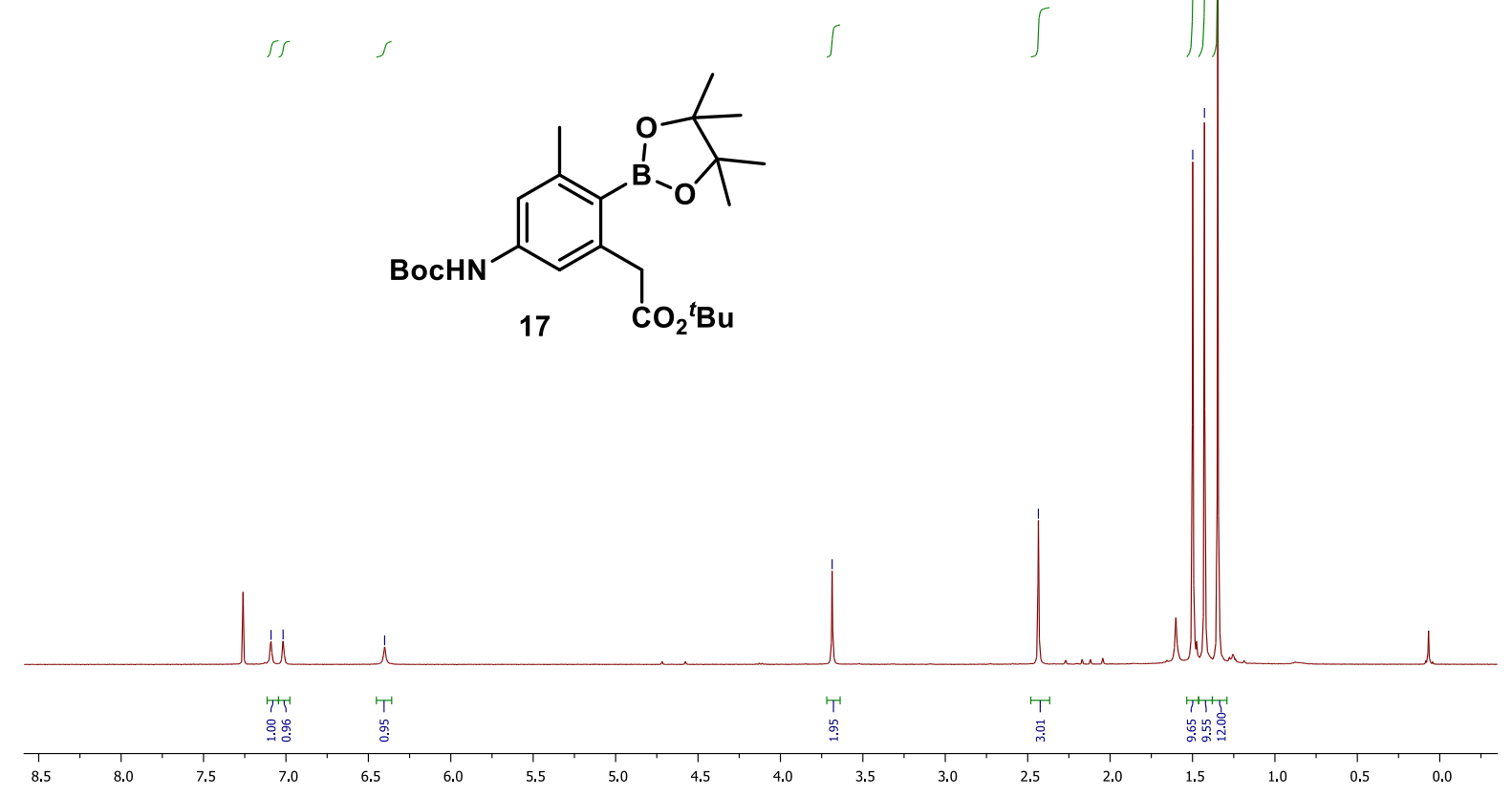

1 In
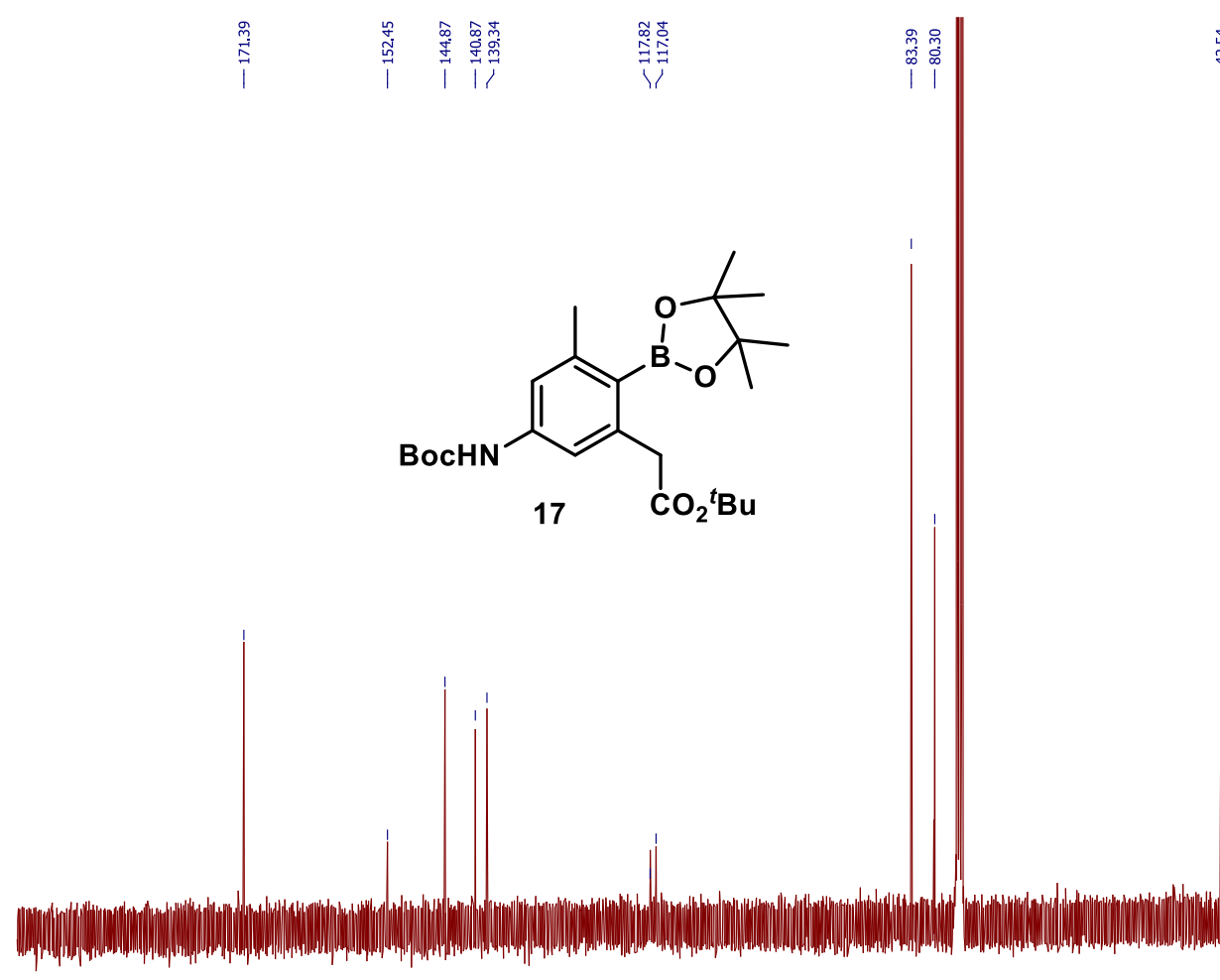

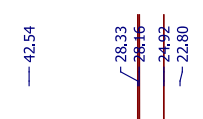

200

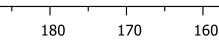

140

${ }_{120}^{1}$ 


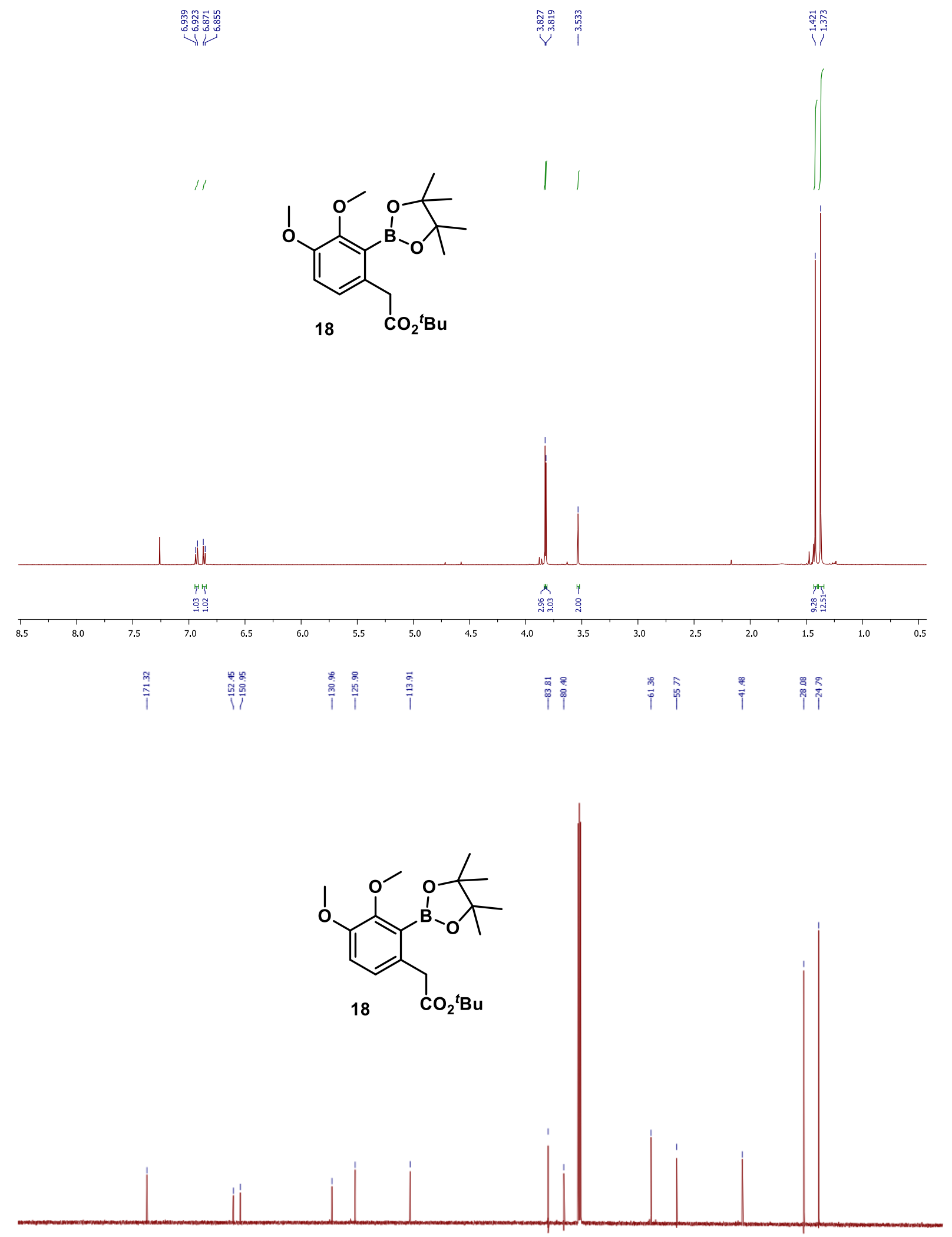



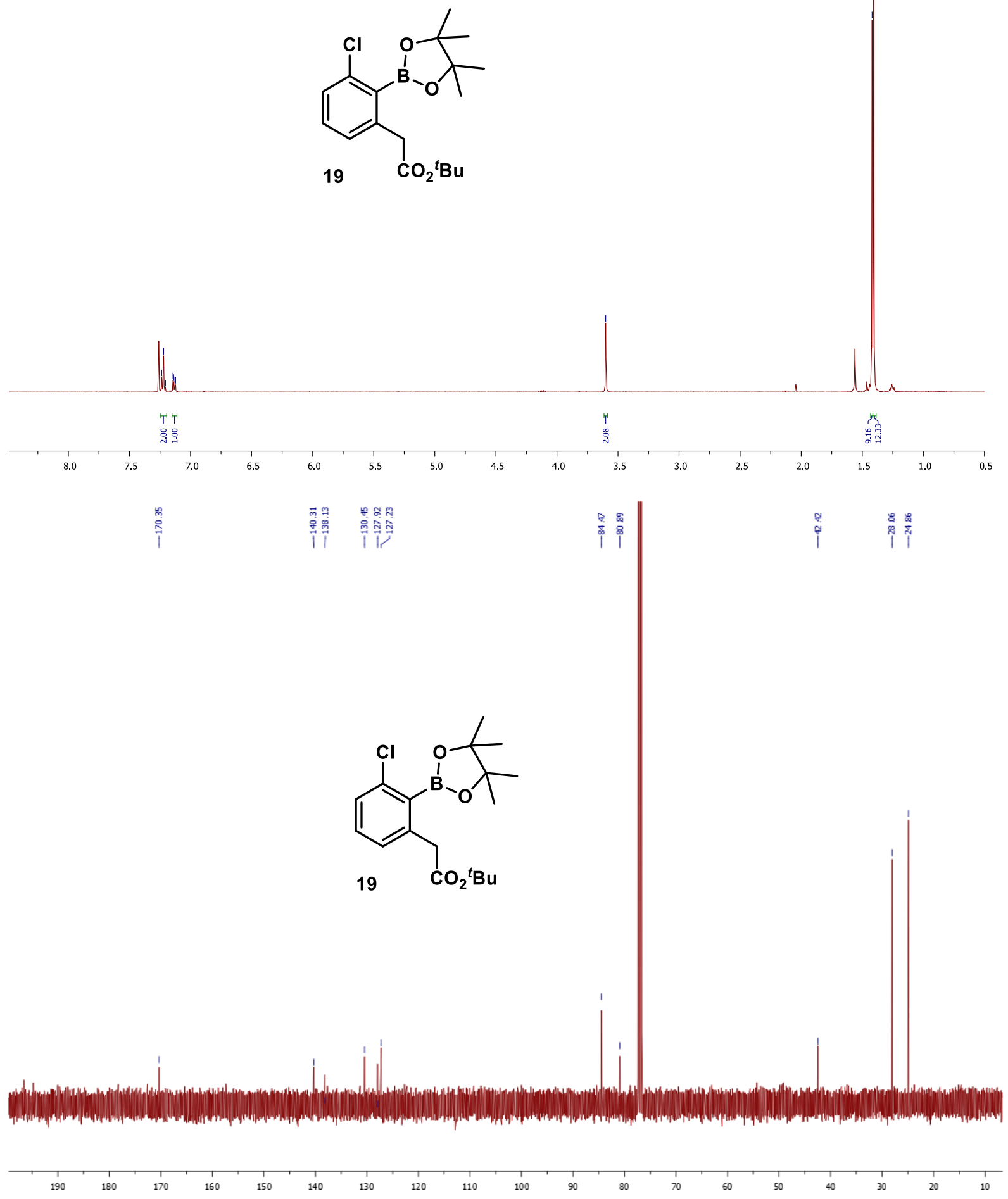

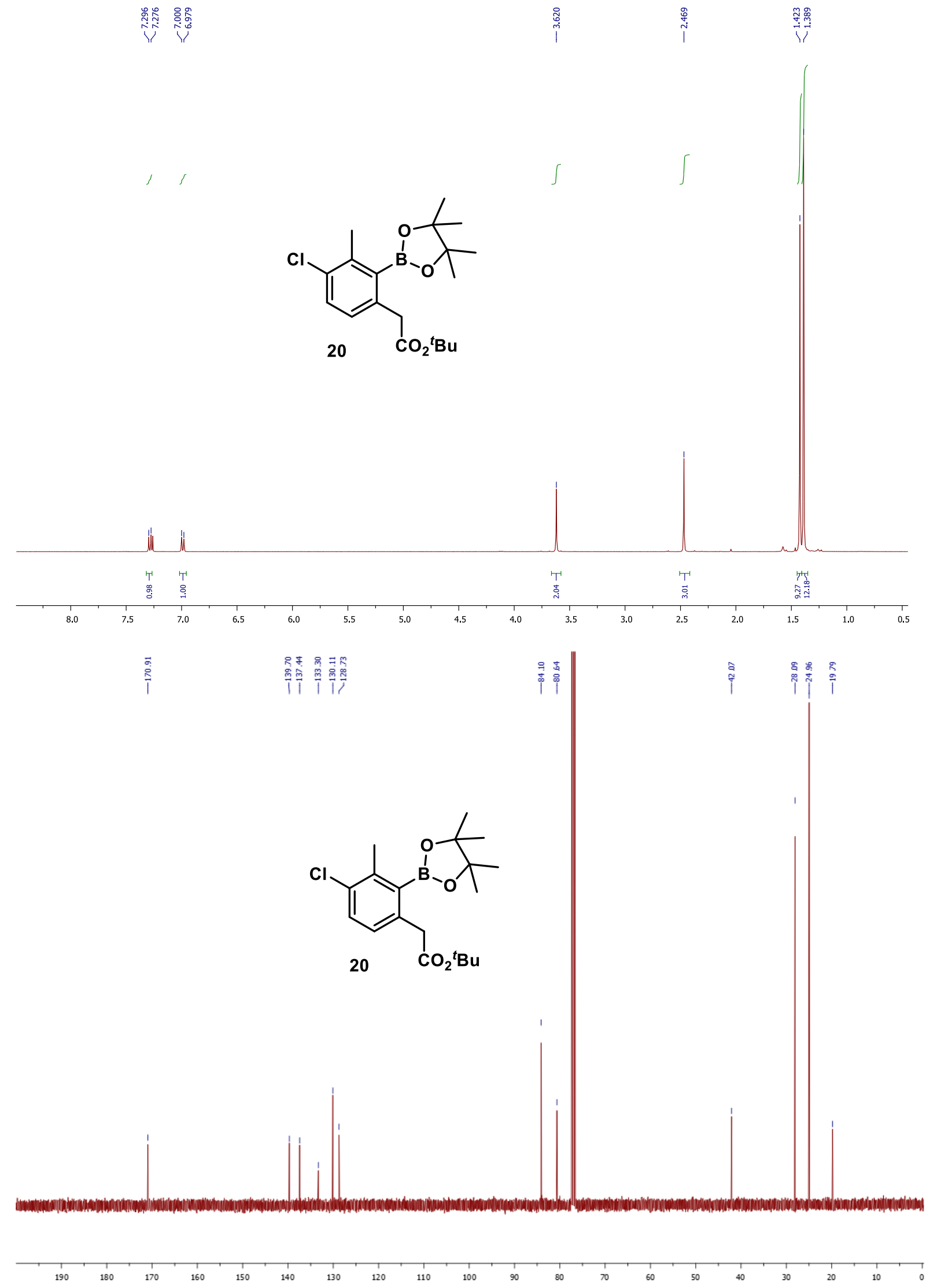

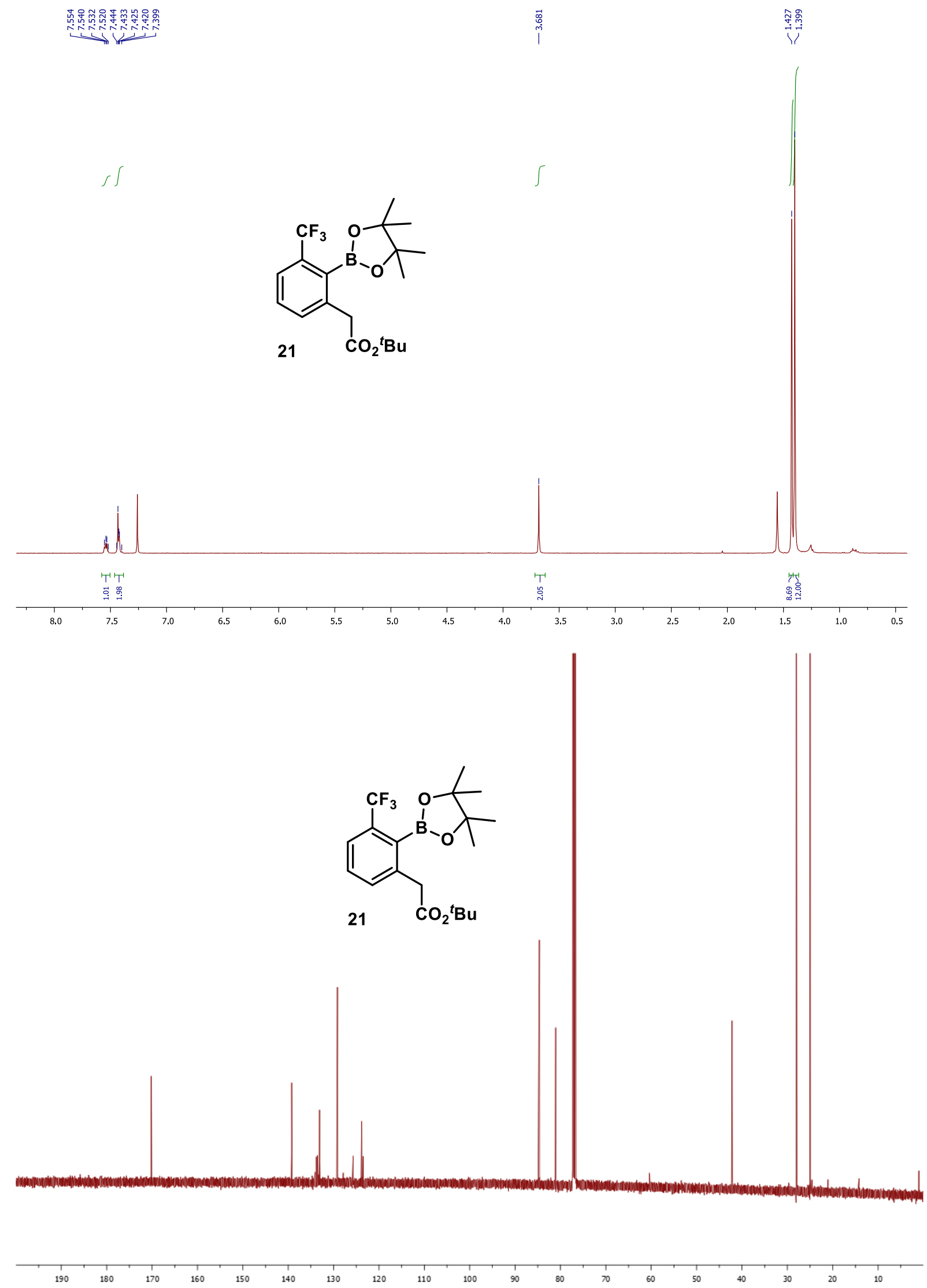


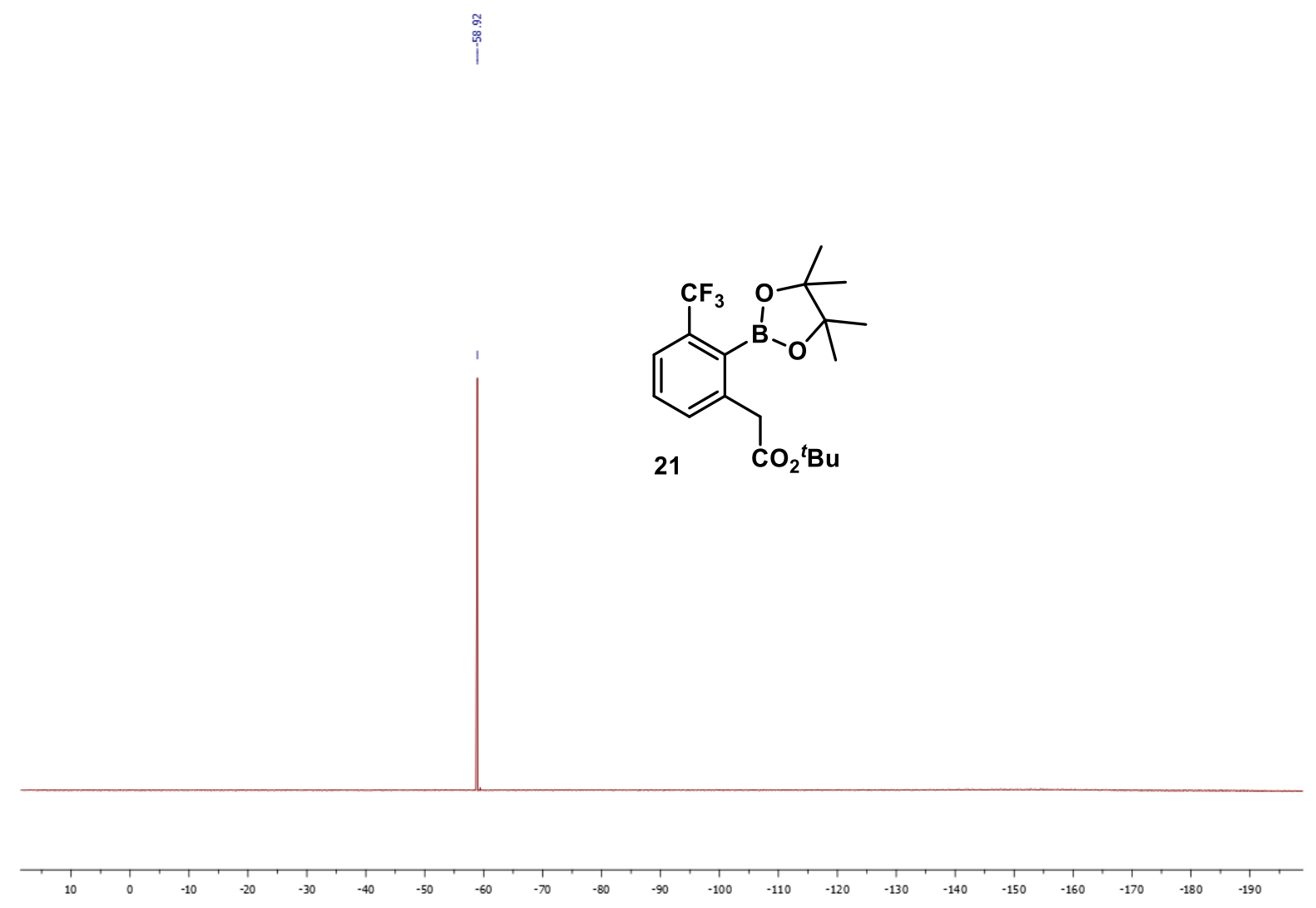



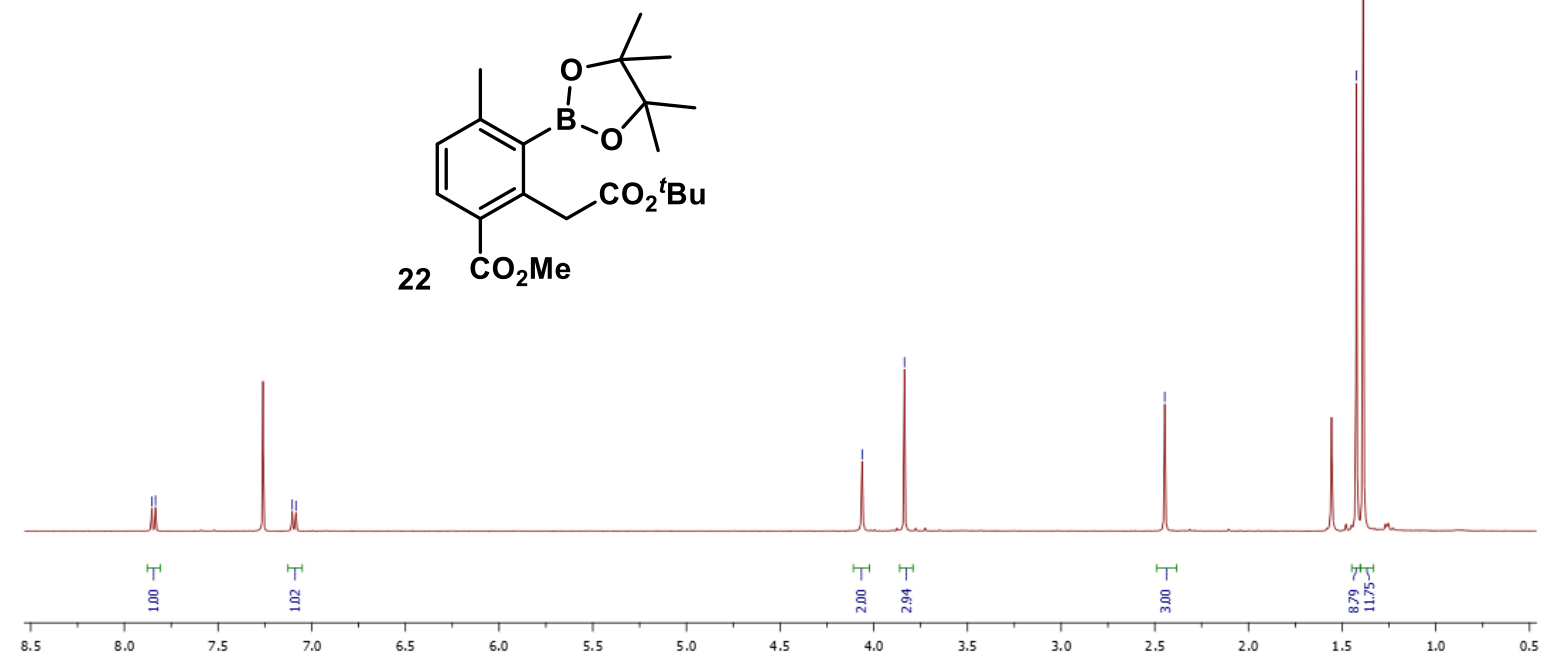

\begin{tabular}{|c|c|c|}
\hline 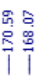 & 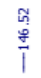 & $\begin{array}{l}\stackrel{+}{0} \\
\stackrel{\sigma}{\vec{l}}\end{array}$ \\
\hline
\end{tabular}

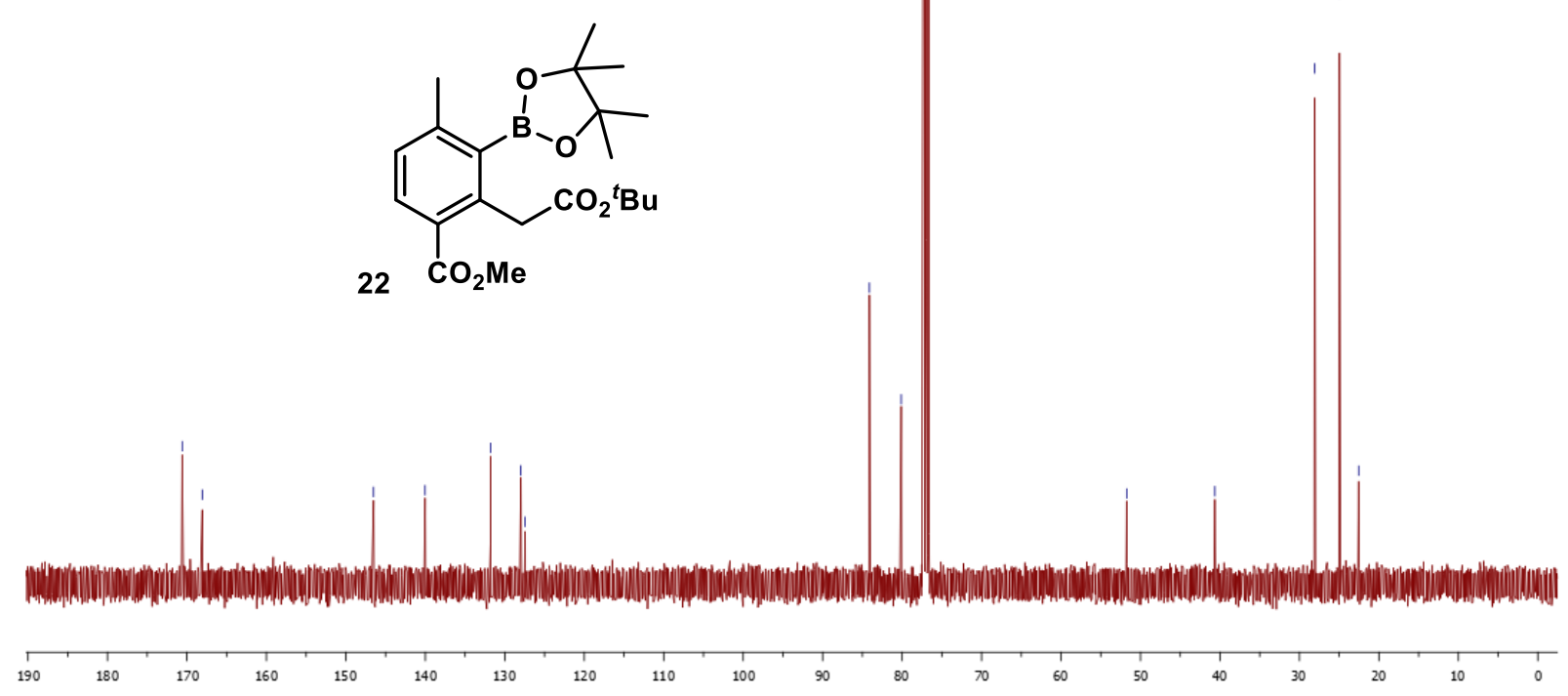



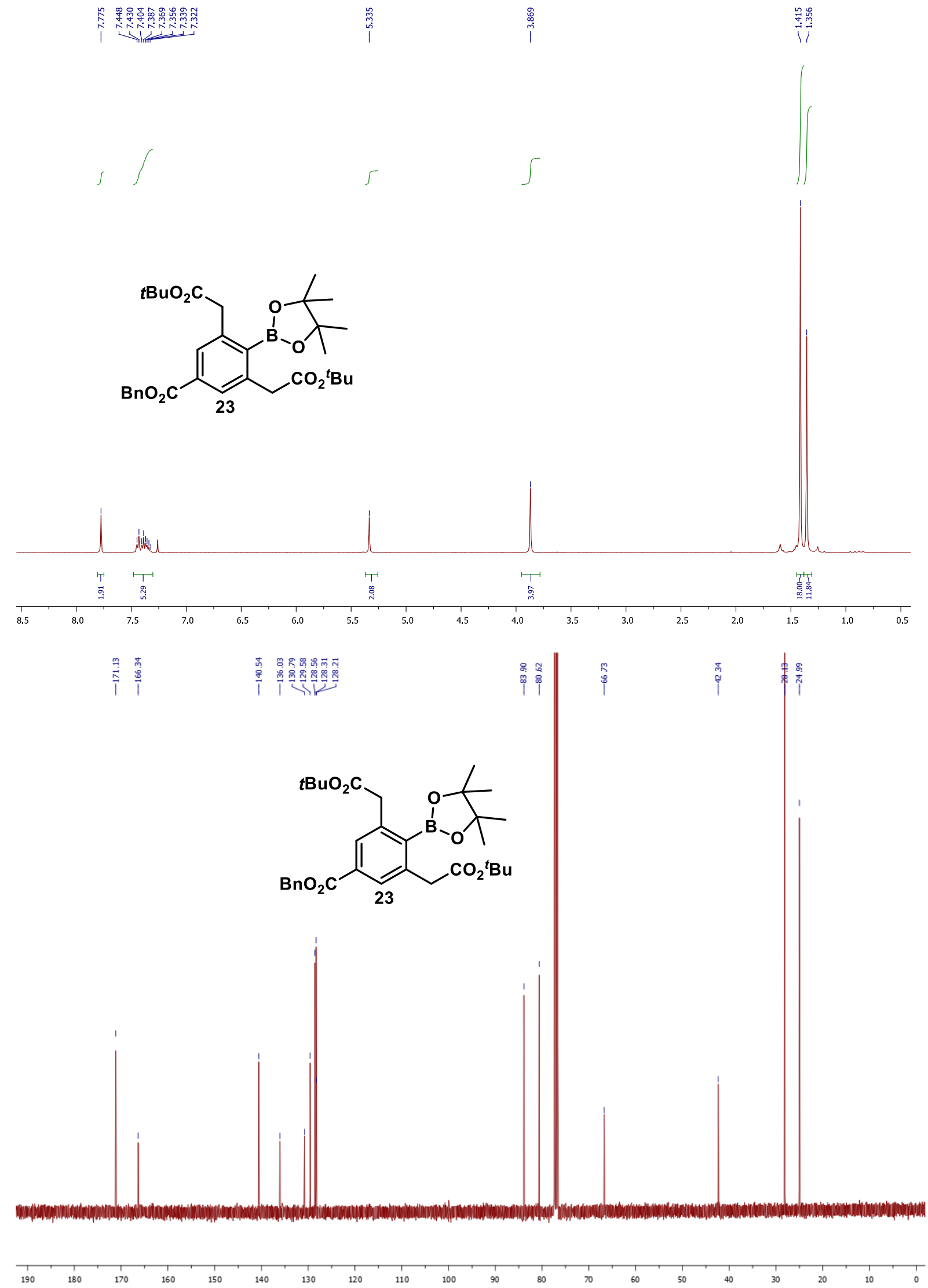

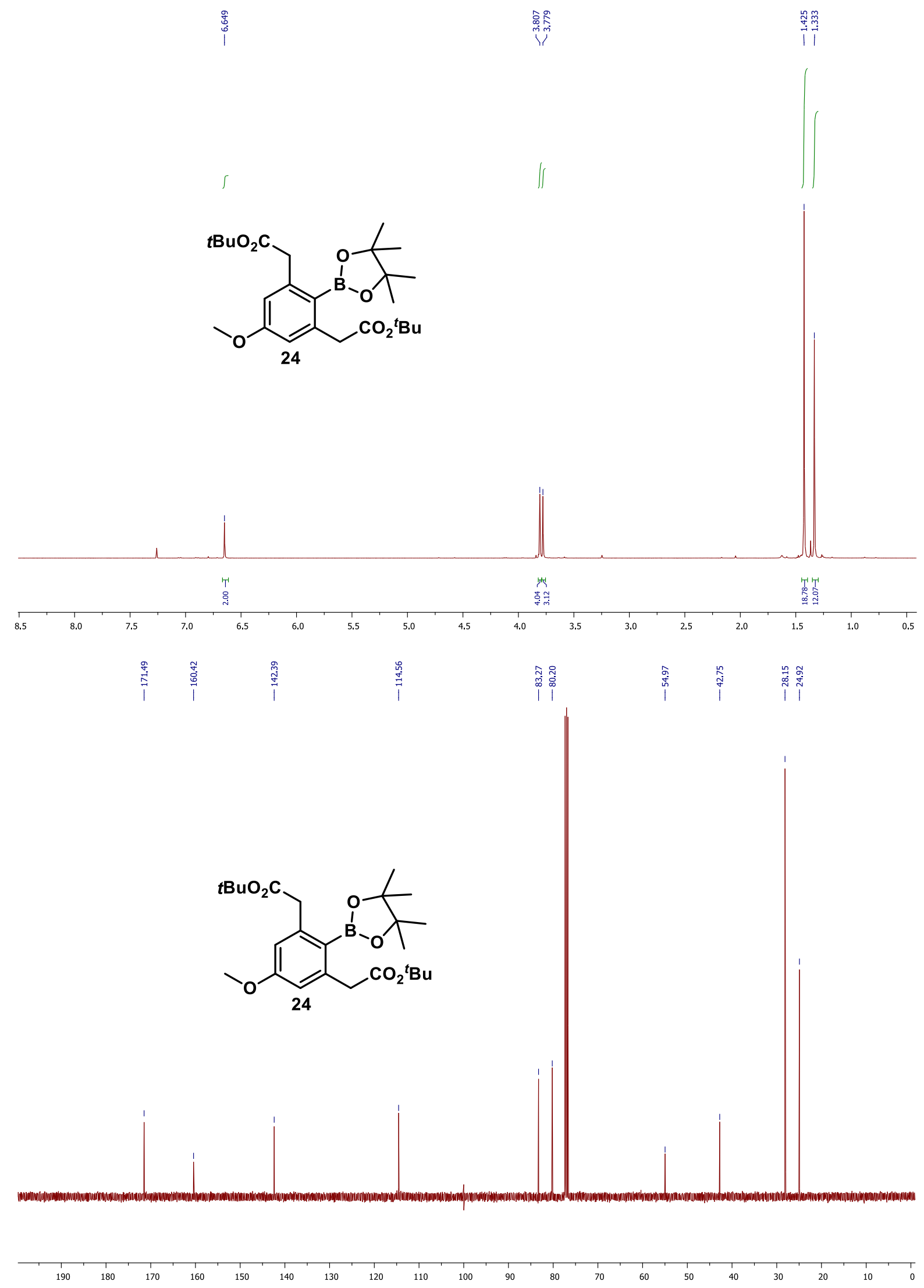


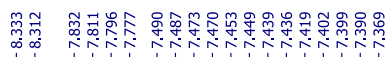
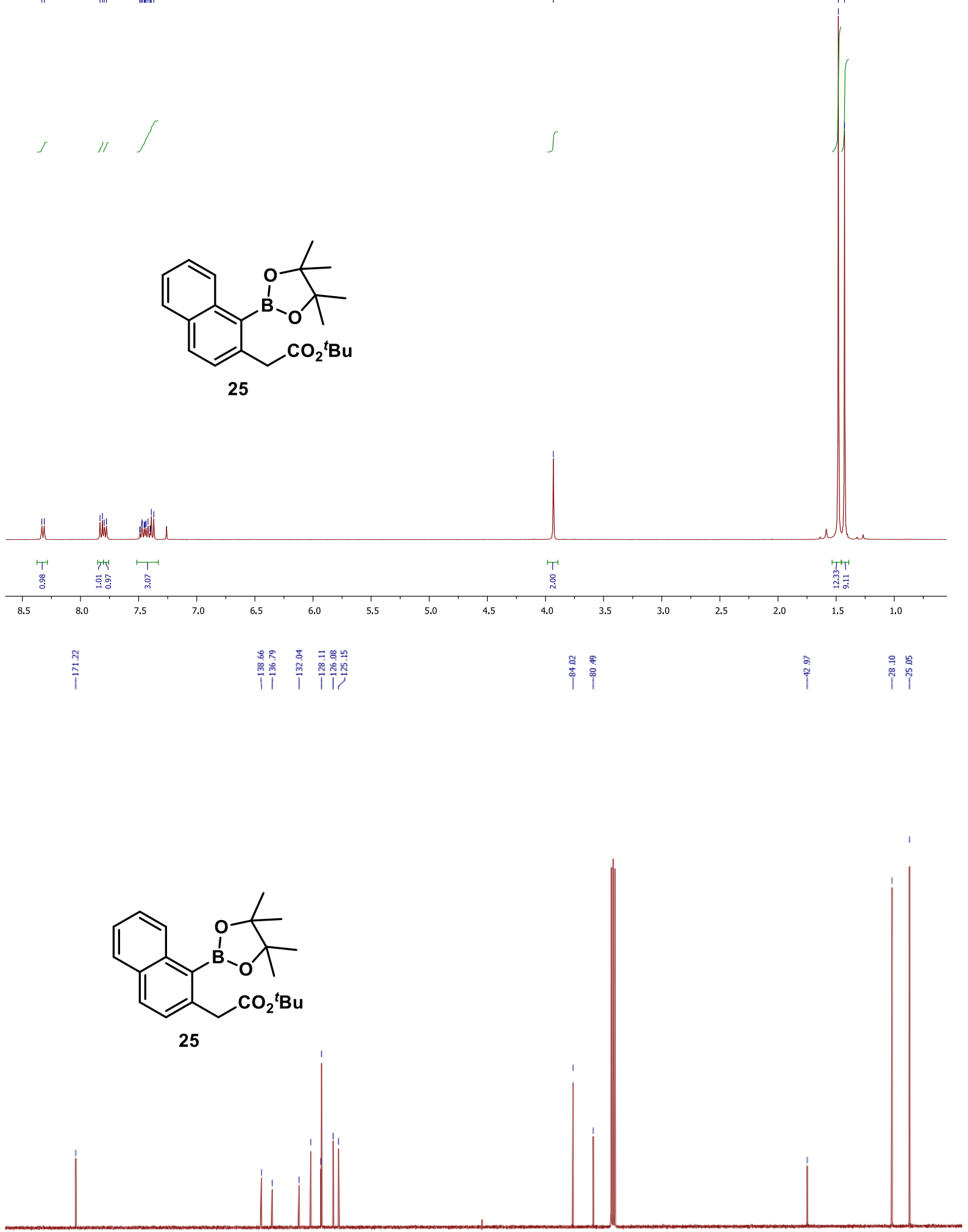


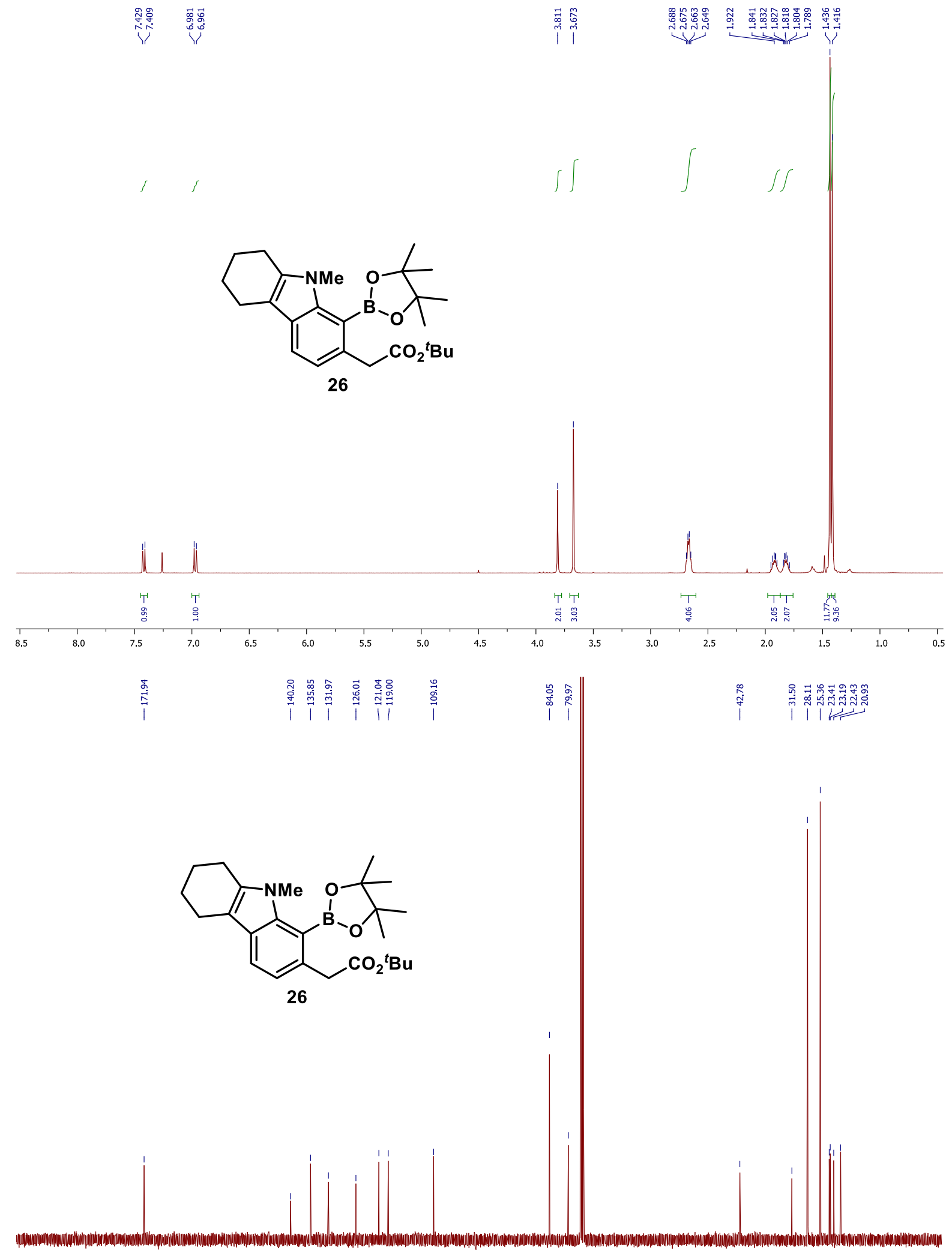

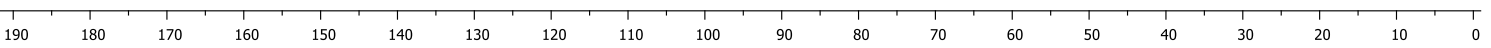




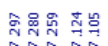

रो

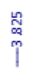

$\mathrm{tBuO}_{2} \mathrm{C}$

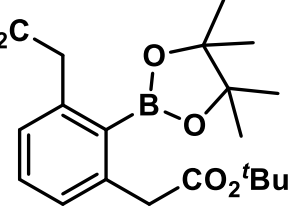

27

是器

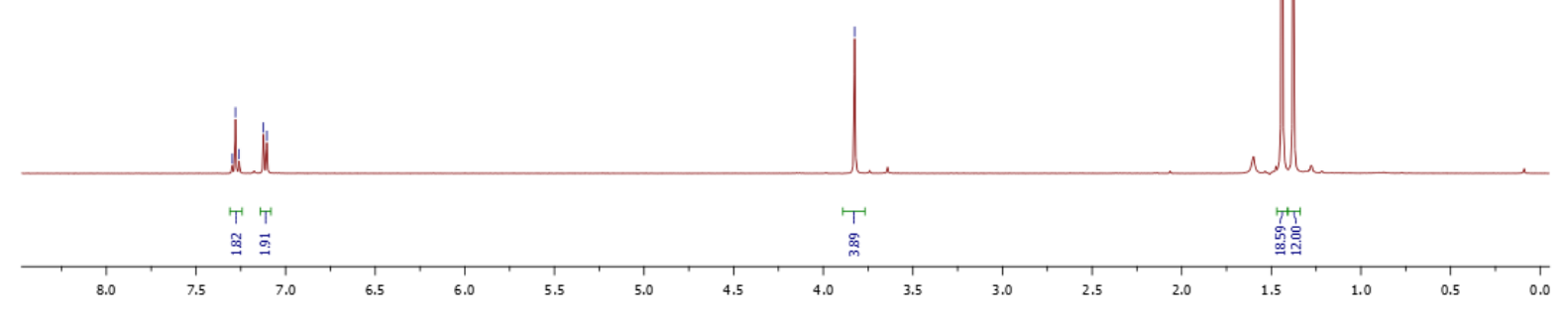

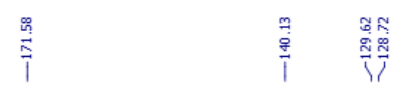

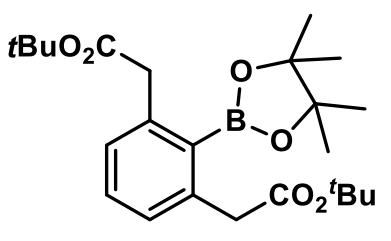

27

190

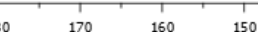

$150 \quad 140 \quad 130 \quad 120$

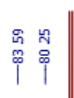

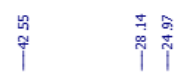

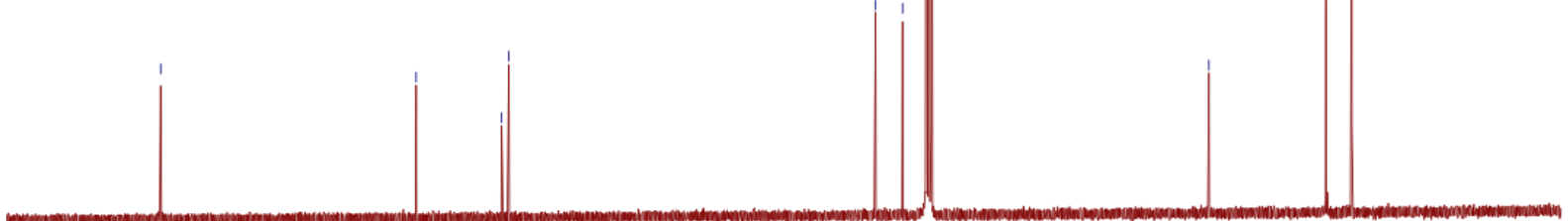




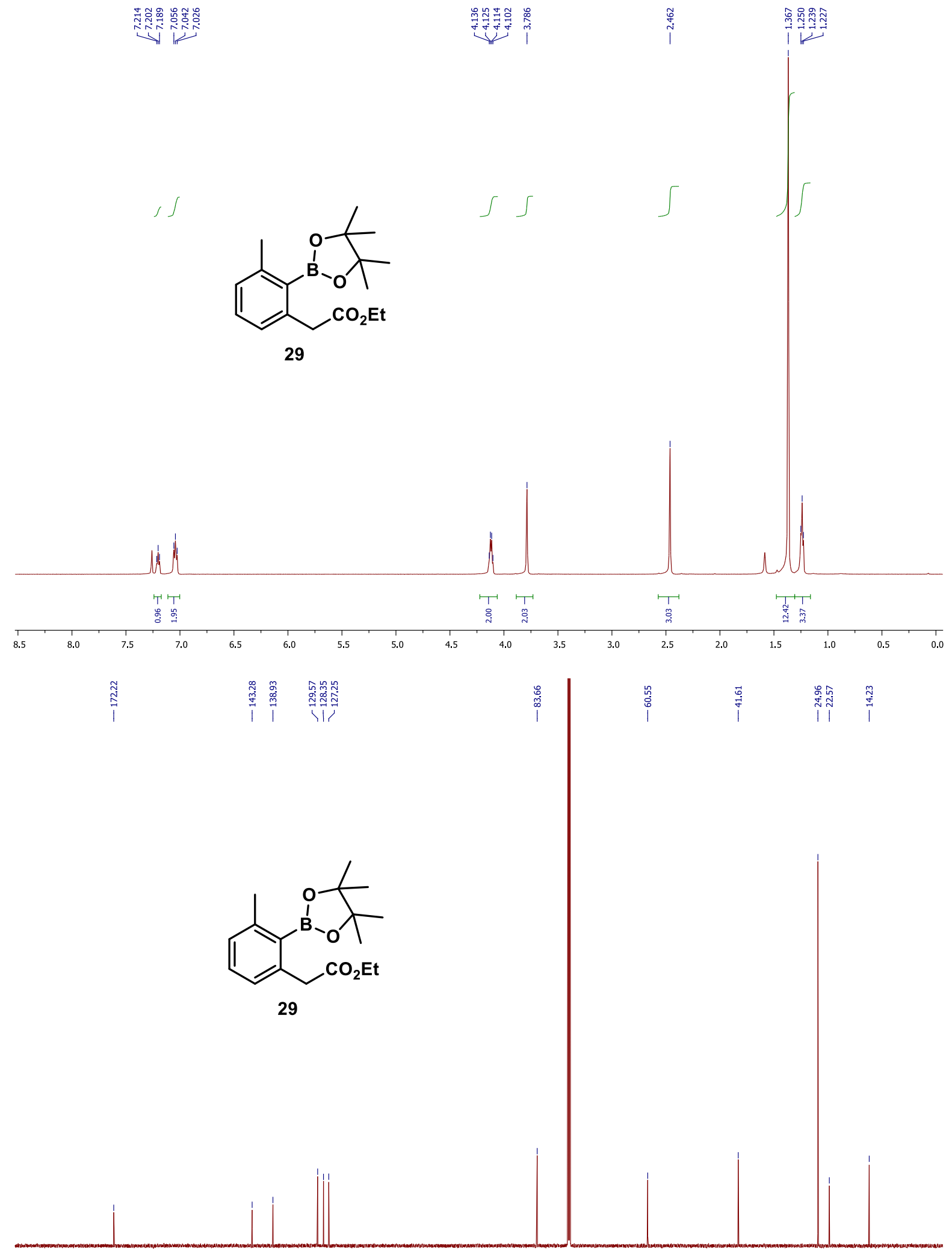



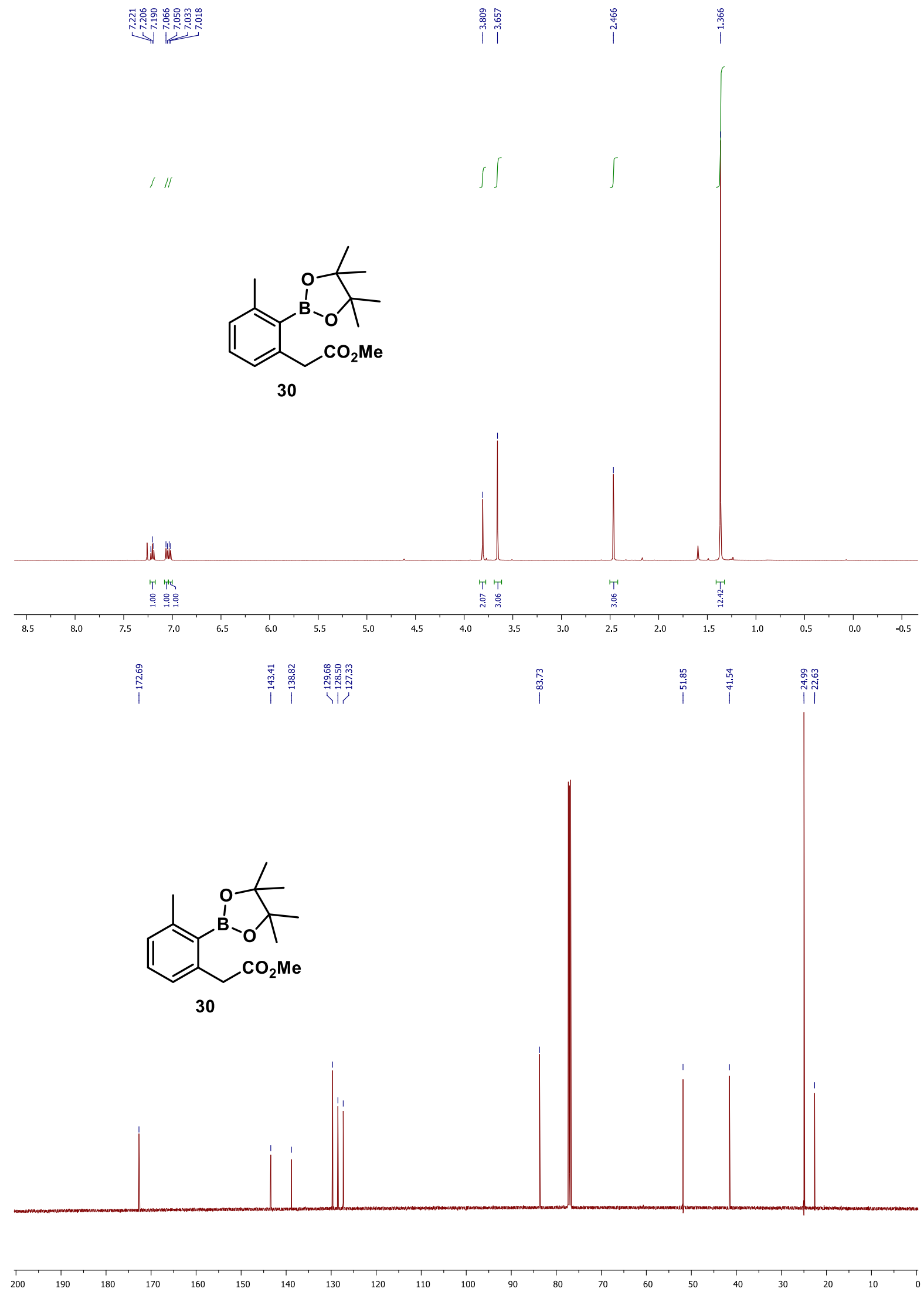

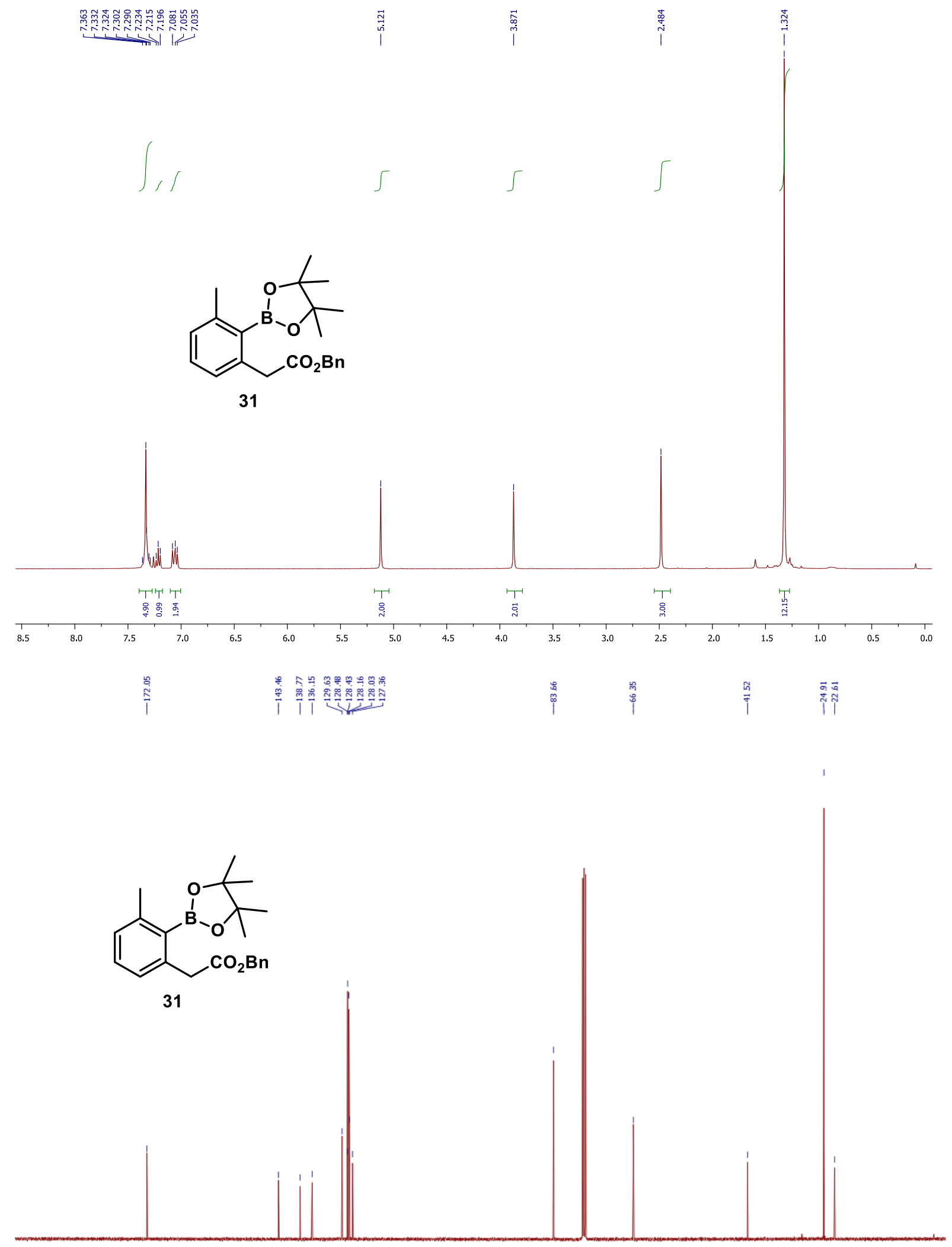


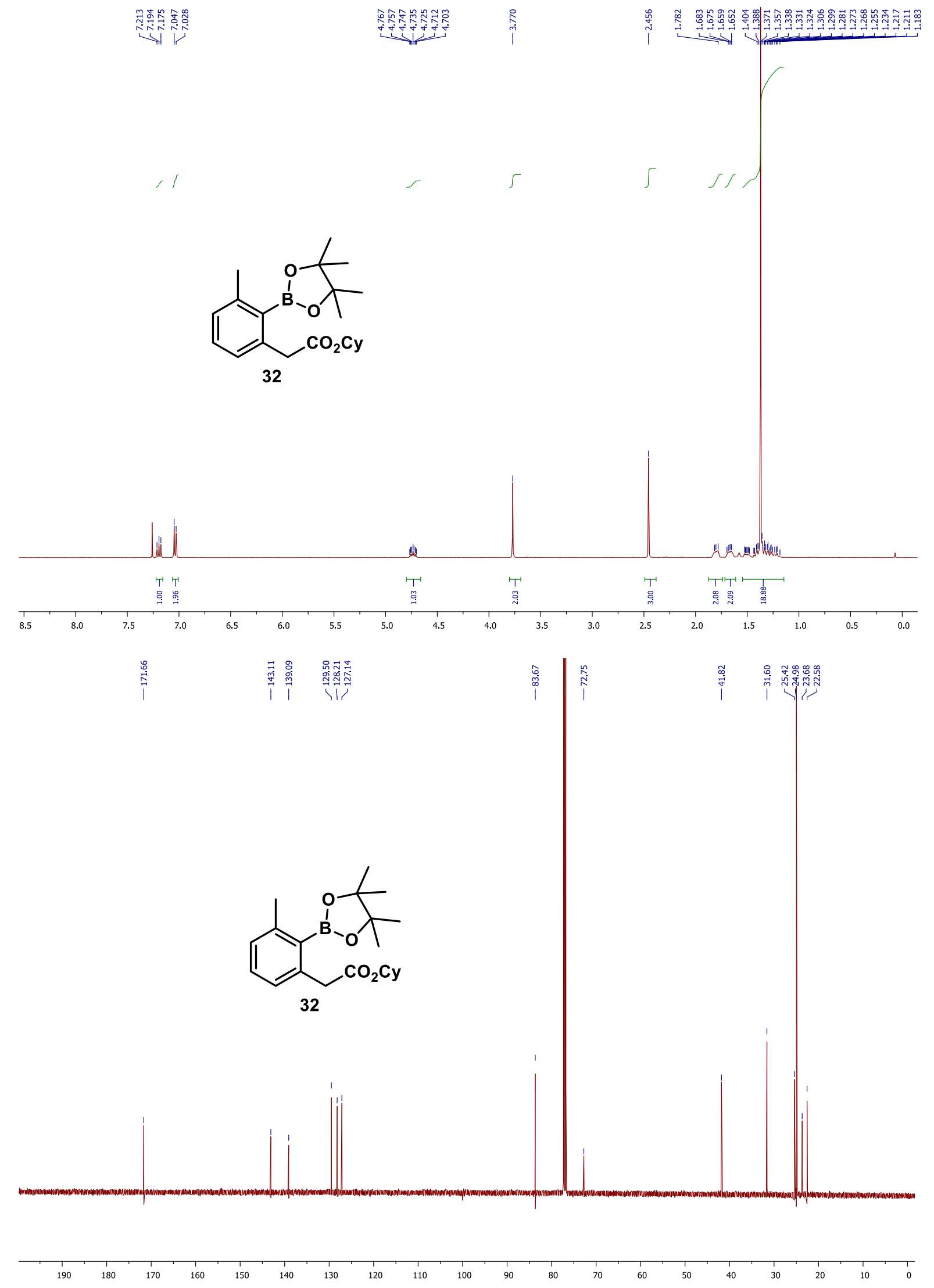



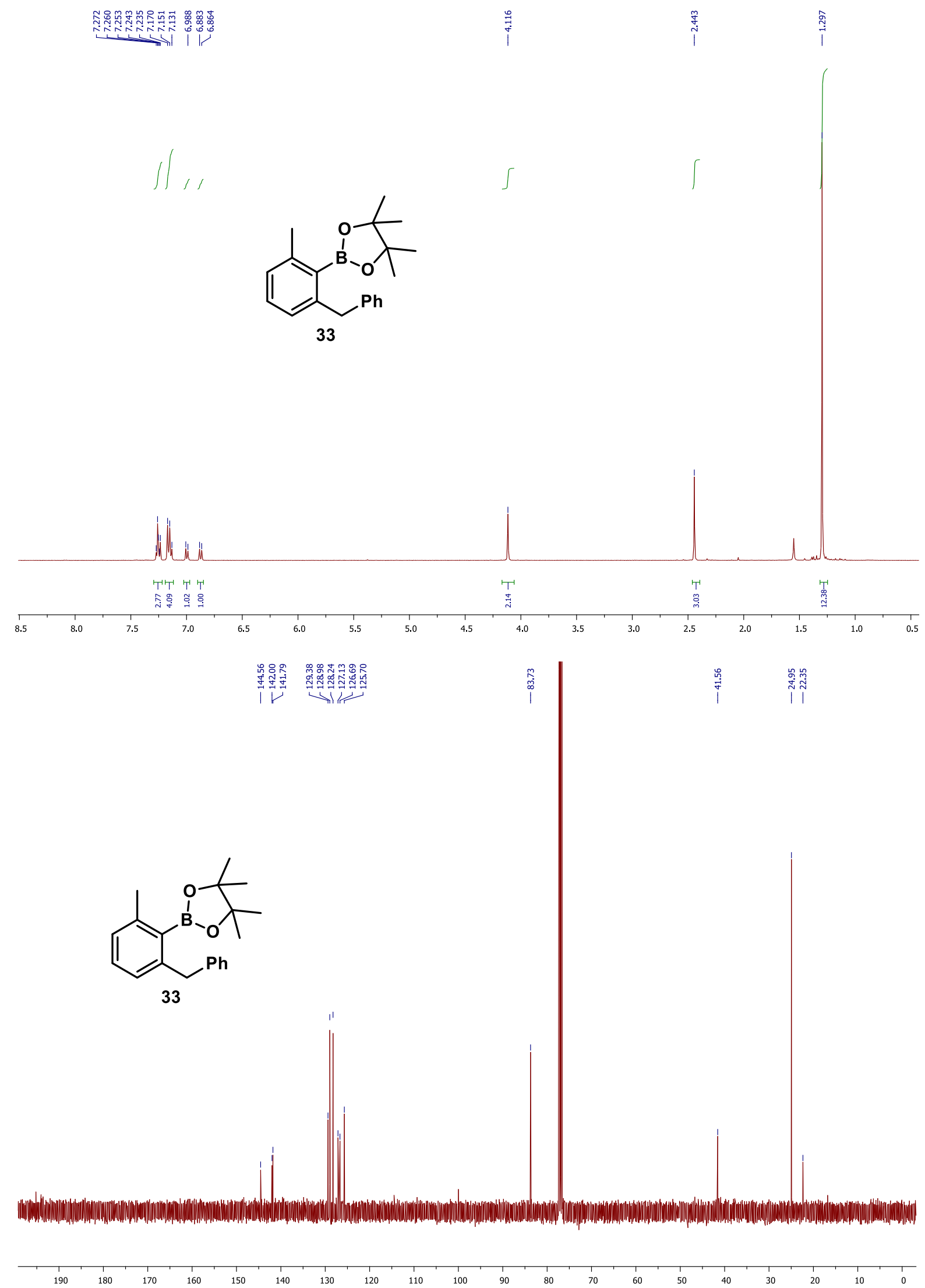

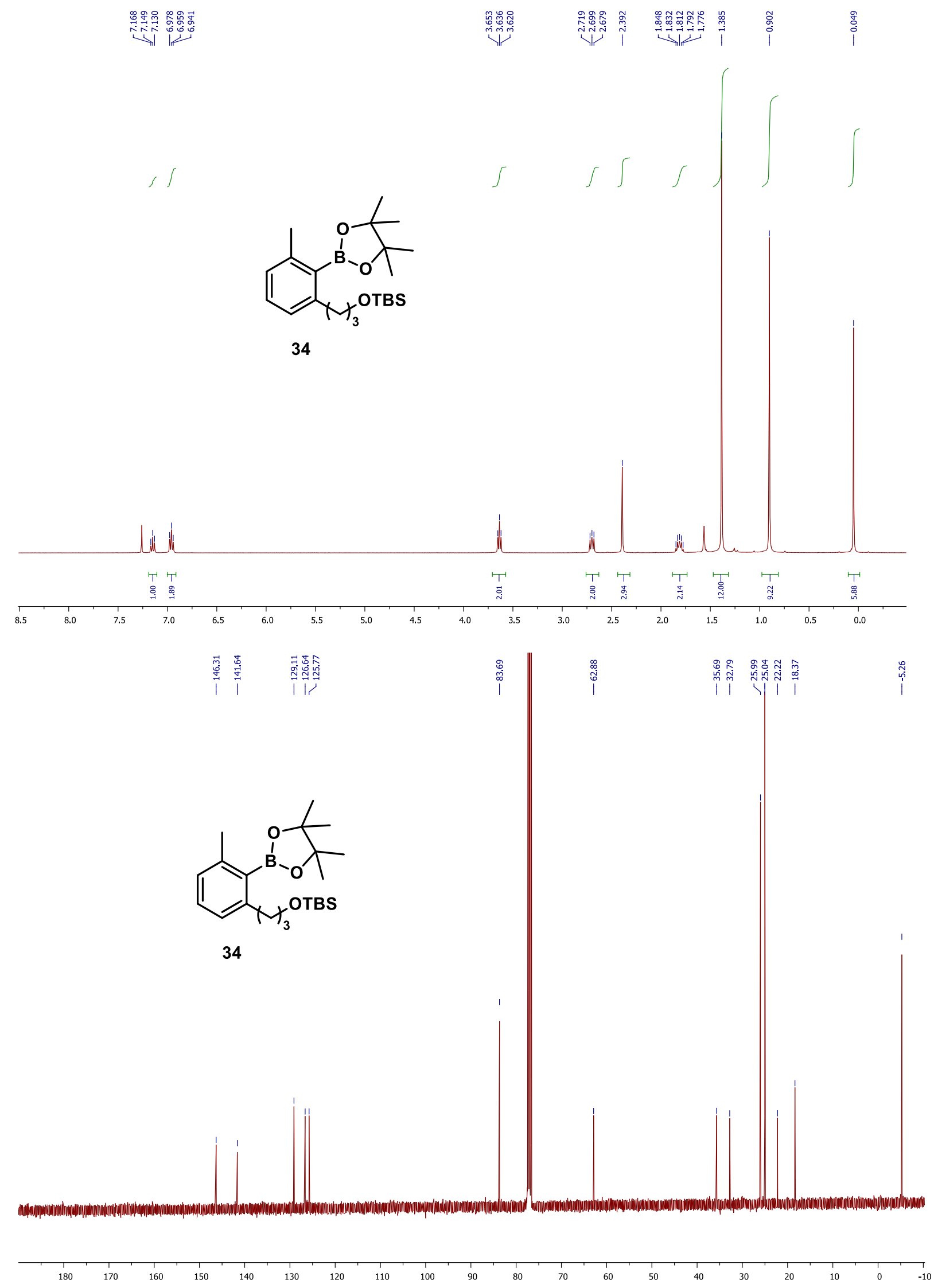


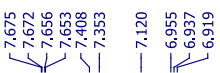

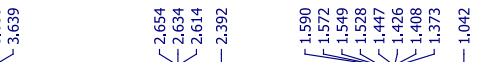

$\iint s$
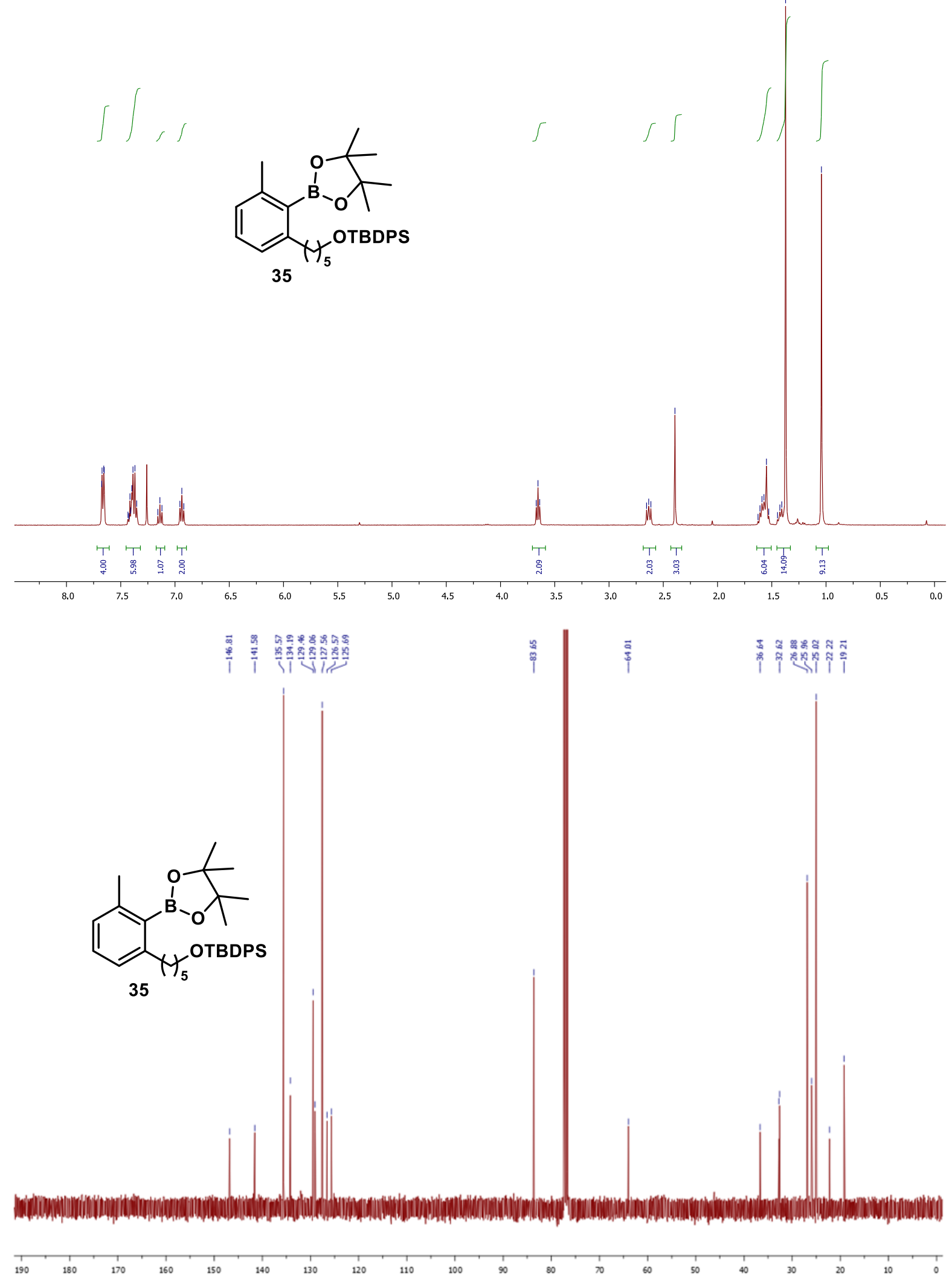


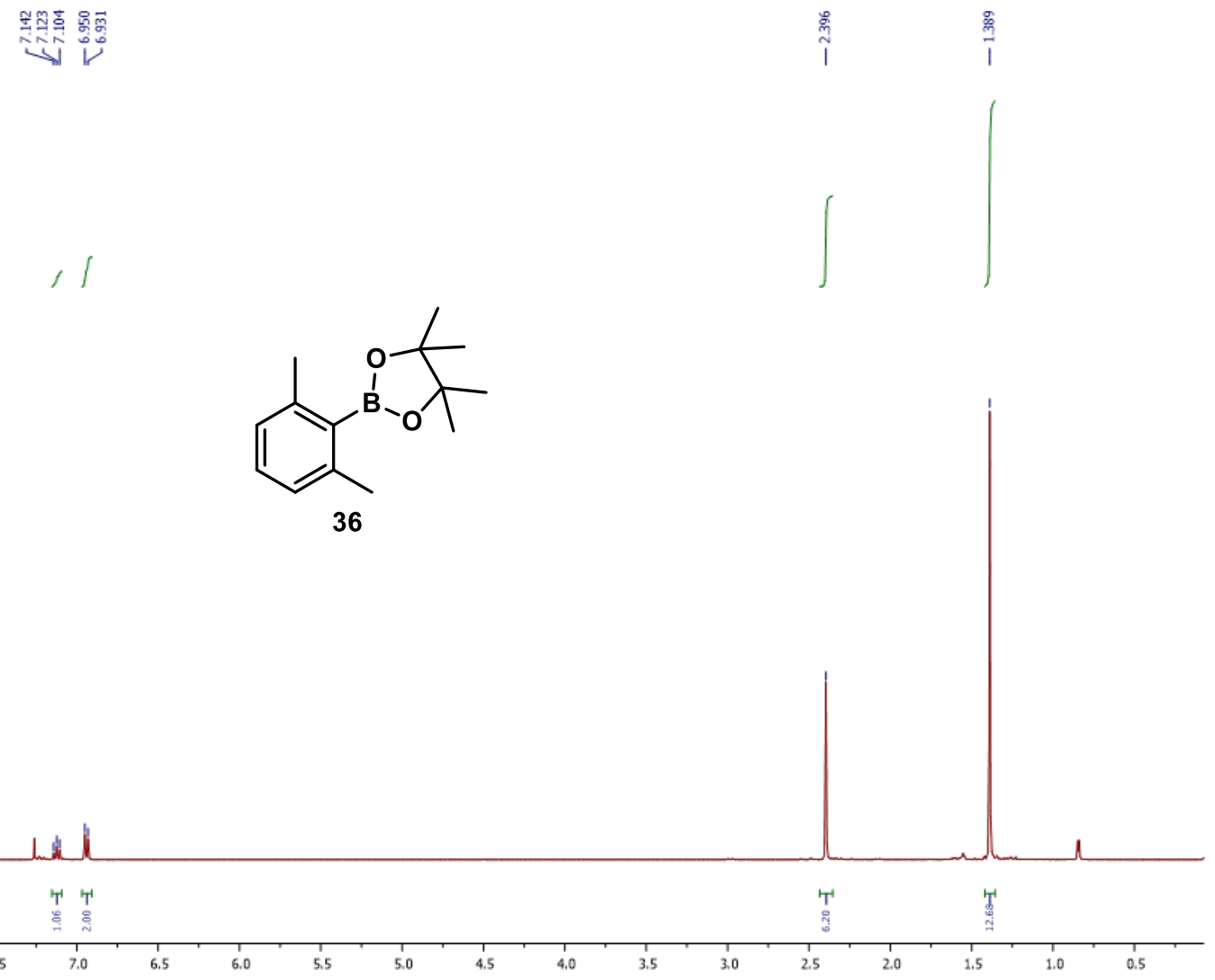




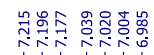

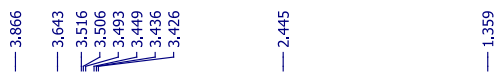

I II

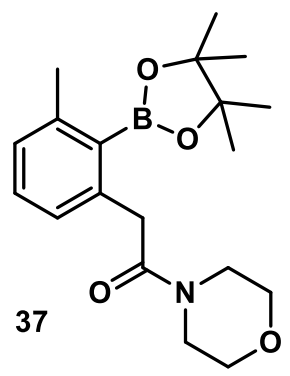

$\iiint \int$

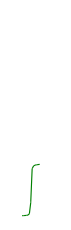

到治

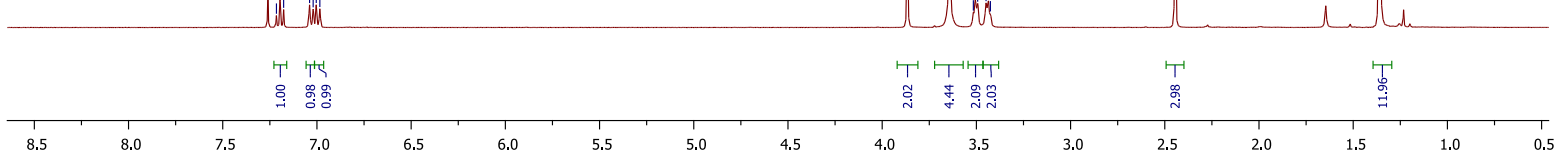

齐

\section{I}

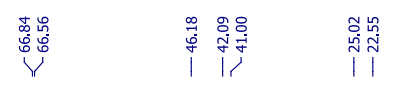

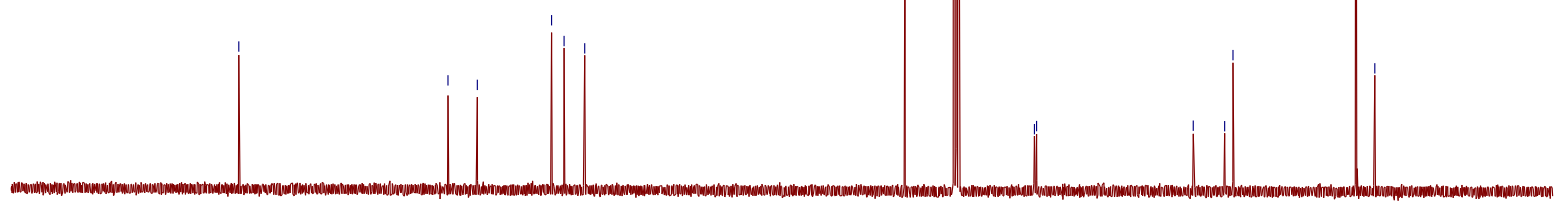




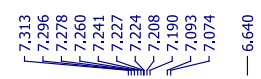

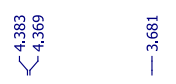
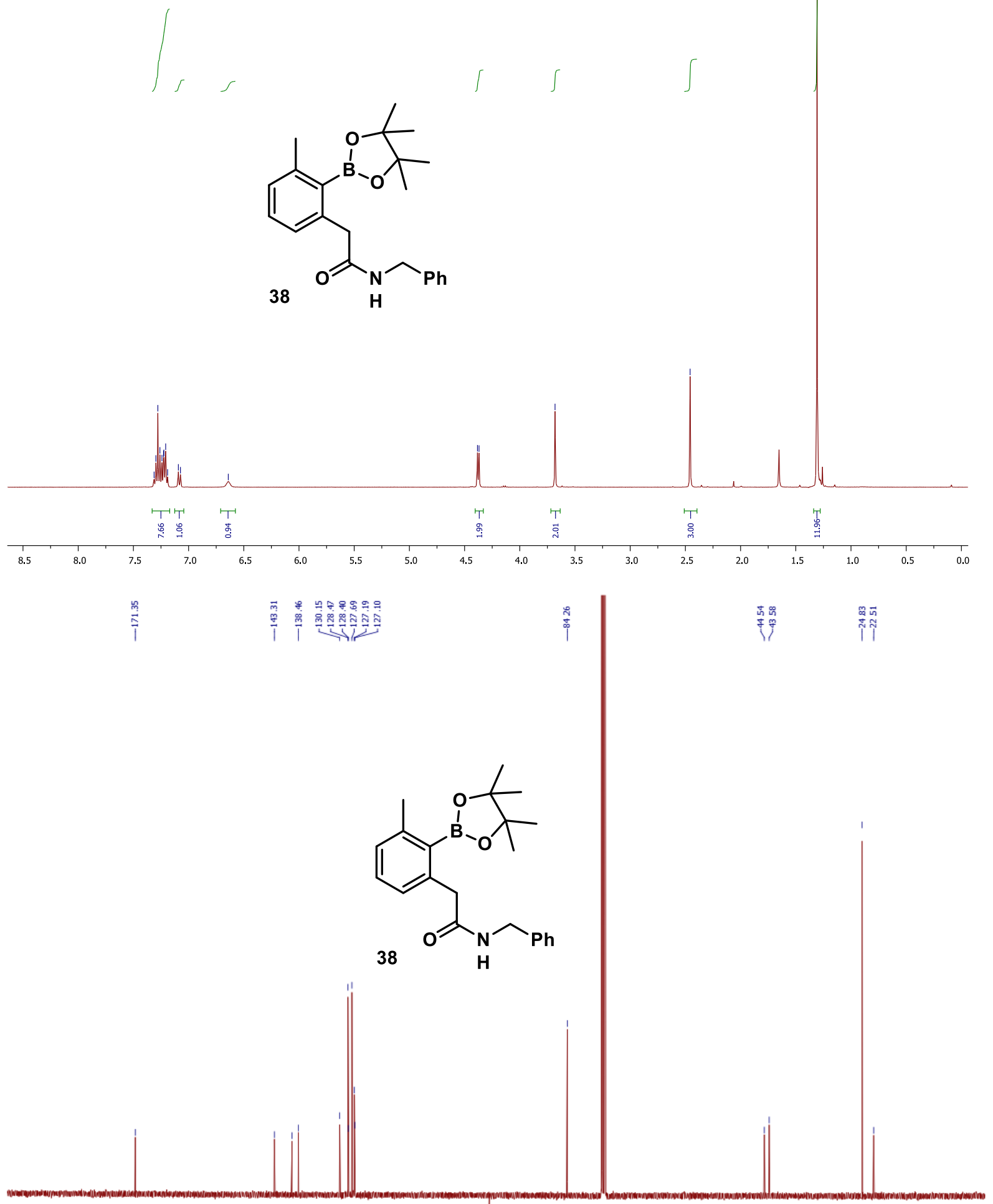

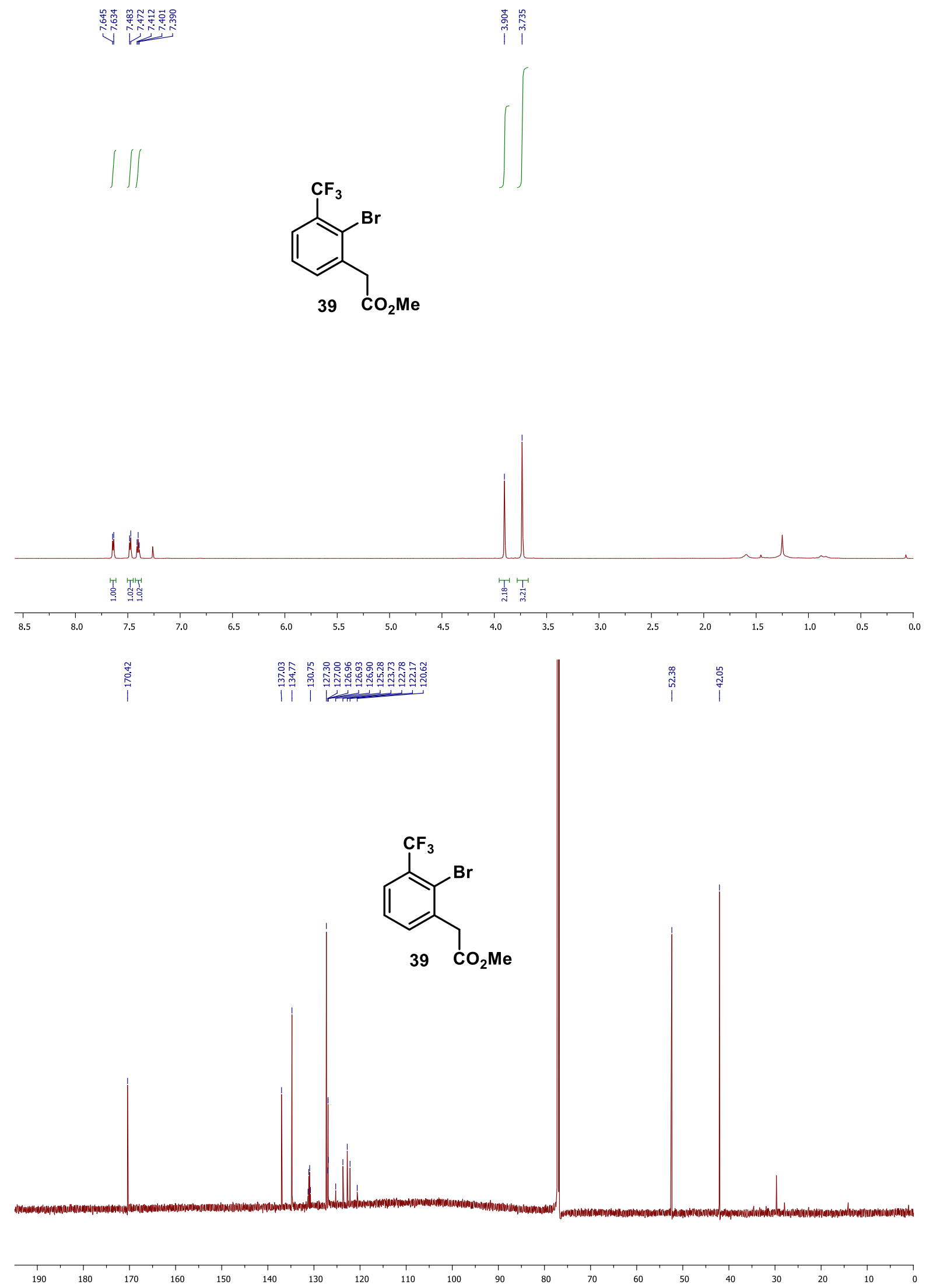


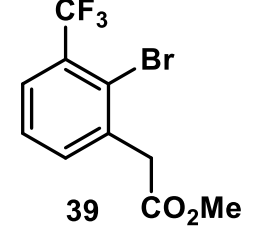

$\begin{array}{llllllllllllllllllllllllll}-45 & -50 & -55 & -60 & -65 & -70 & -75 & -80 & -85 & -90 & -95 & -100 & -105 & -110 & -115 & -120 & -125 & -130 & -135 & -140 & -145 & -150 & -155\end{array}$ 

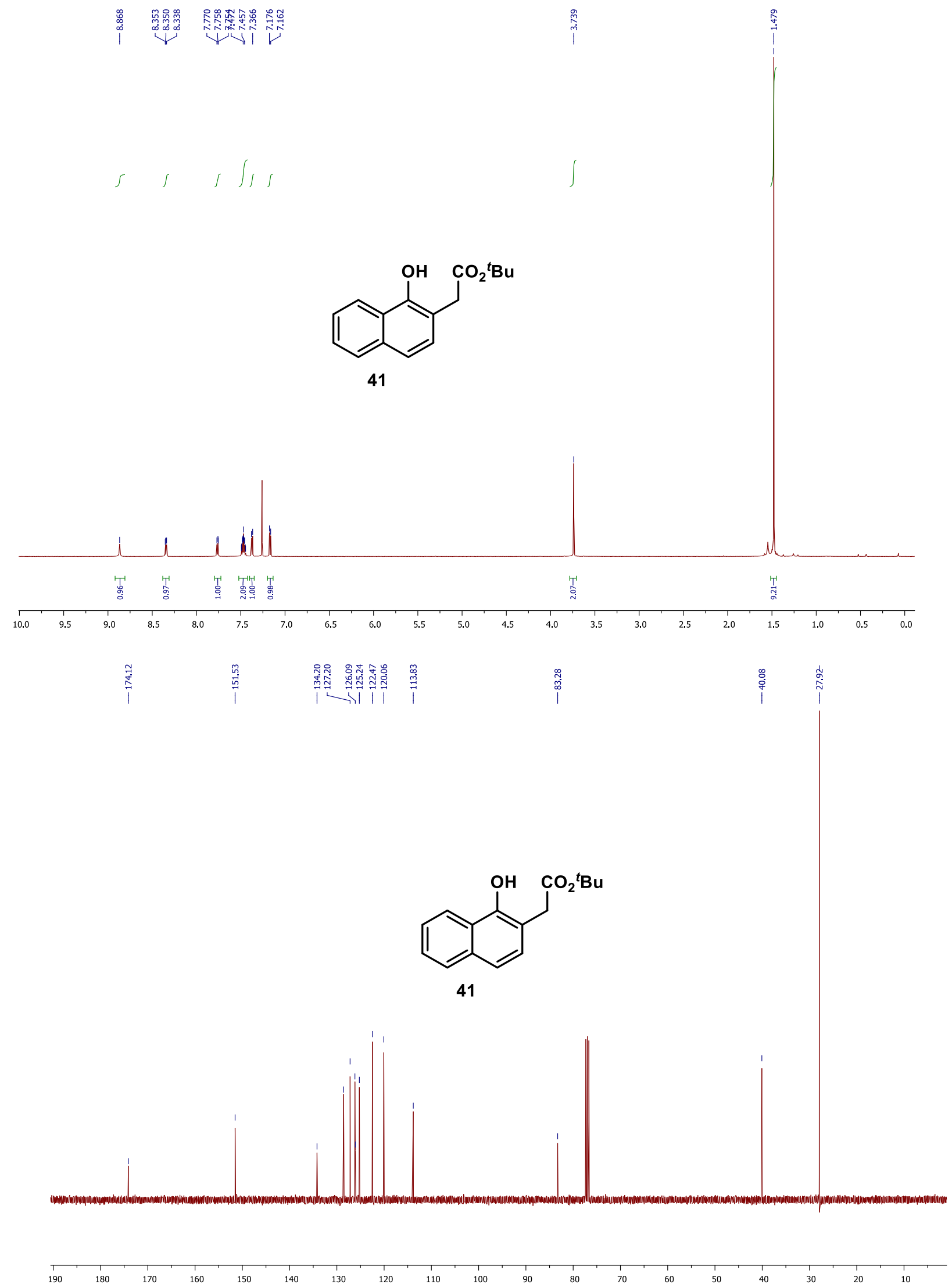

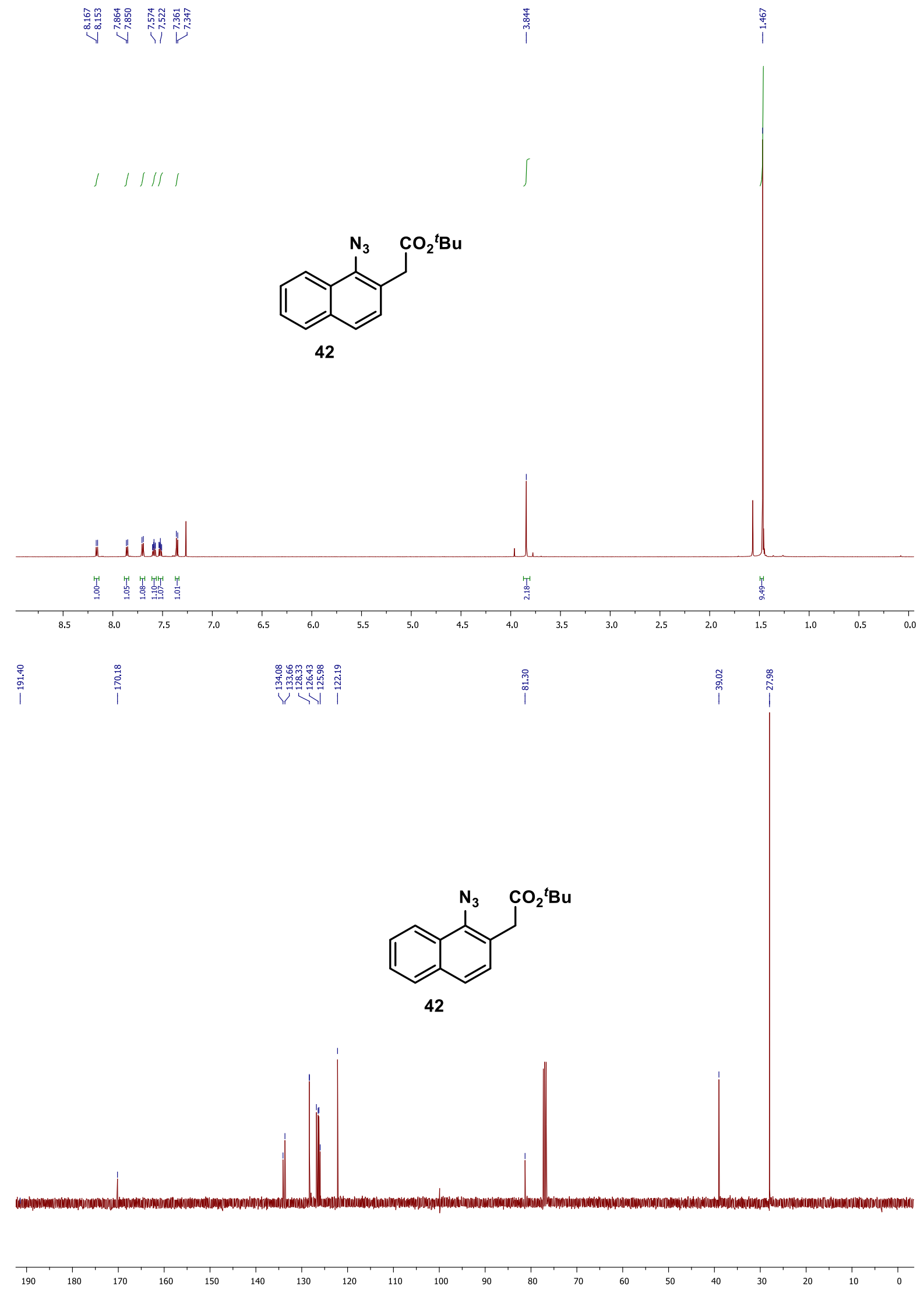

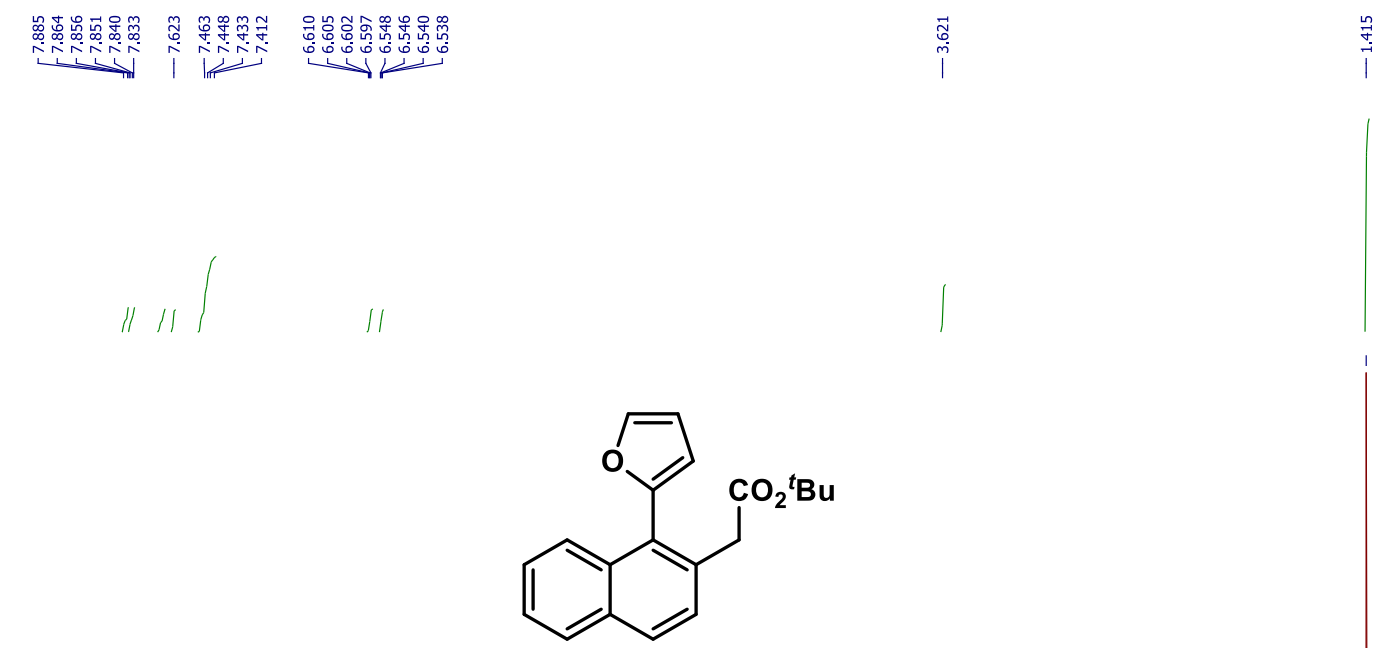

43
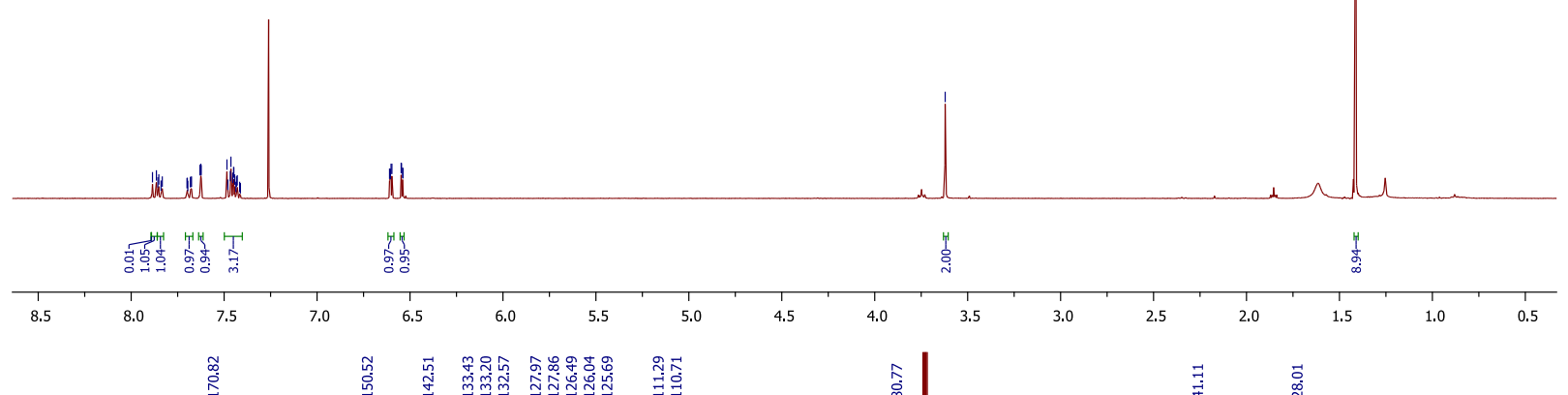

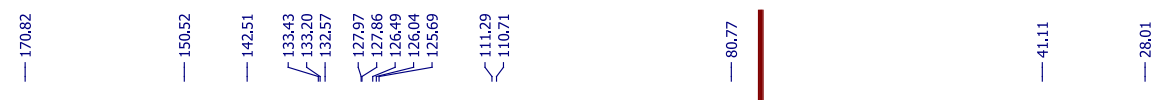

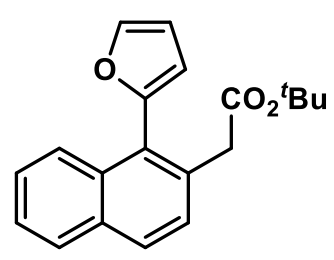

43

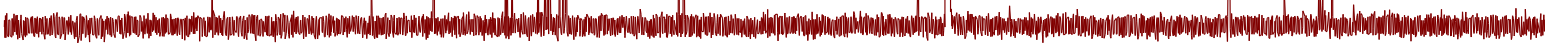




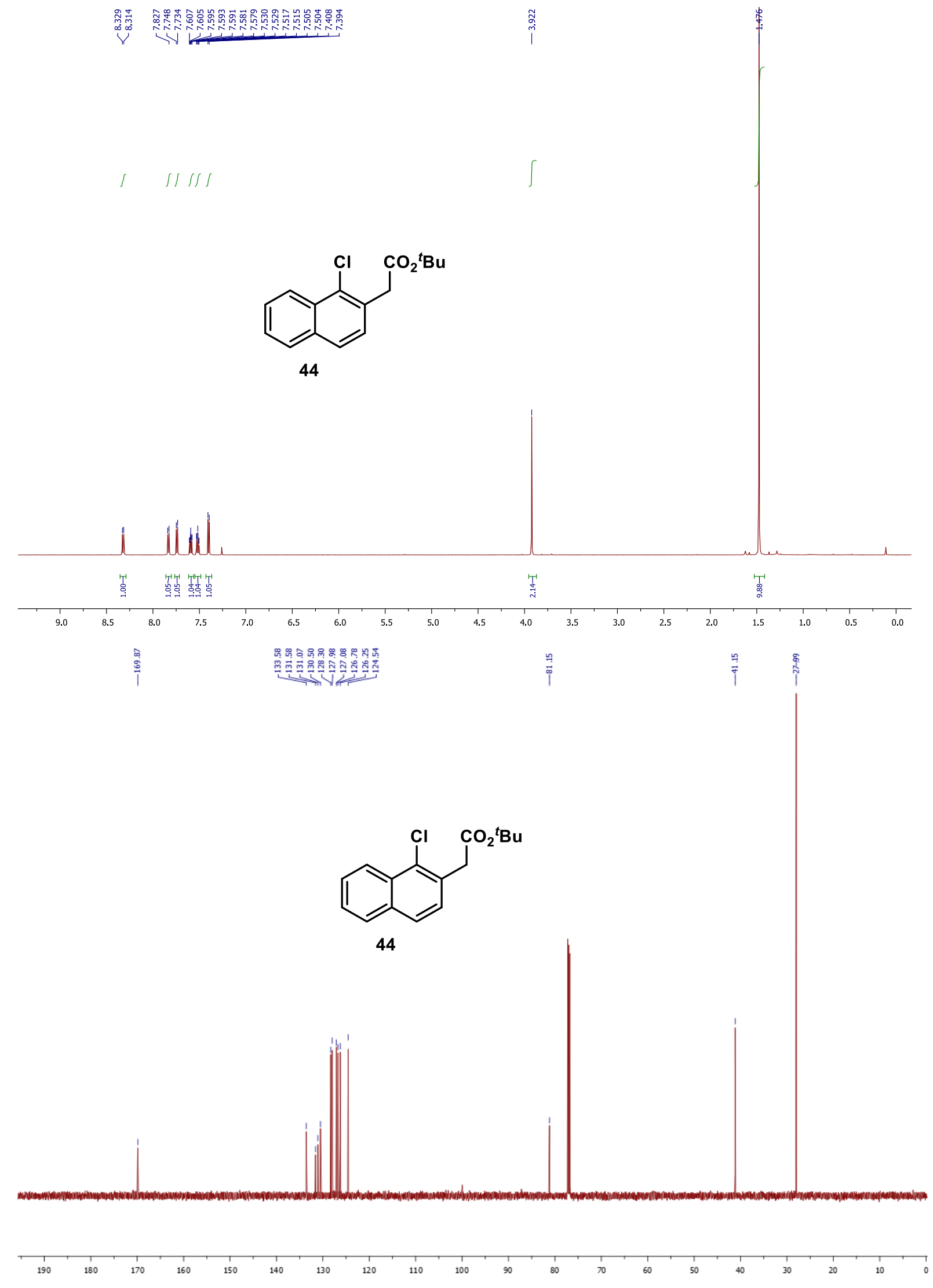




\section{REFERENCES}

1. Sonobe, T.; Oisaki, K.; Kanai, M. Chem. Sci. 2012, 3, 3249.

2. (a). Shama, P.; Srinivasa Reddy, T.; Thummuri, D.; Senwar, K. R.; Shankaraiah, N. et al. Eur. J. Med. Chem. 2016, 124, 621. (b). Lee, D.; Kim, D.; Lee, S.; et al. Molecules 2015, 20, 19984-20013.

3. Yokoyama, A.; Maruyama, T.; Tagami, K.; Masu, H.; Katagiri, K.; Azumaya, I.; Yokozawa, T. Org. Lett. 2008, 10, 3207.

4. Kiehne, U.; Lutzen, A. Eur. J. Org. Chem. 2007, 5703-5711.

5. Zhang, C.; Zheng, M.; Li, Y.; Yang, S. et al. J. Med. Chem. 2015, 58, 3957.

6. Garcia, L. A.; Arias, E.; Moggio, I. et al. Polymer, 2011, 52, 5326.

7. Lopez-Iglesia, M.; Busto, E.; Gotor, V.; Gotor-Fernandez, V. J. Org. Chem. 2012, 77, 8049.

8. Lesk, A.; Nudelman, A. Synth. Commun. 1999, 29, 37-41.

9. Boureghda, C.; Mace, A.; Berree, F.; Roisnel, T.; Debache, A.; Carboni, B. Org. Biomol. Chem. 2019, 17, 5789.

10. Shi, H.; Babinski, D. J.; Ritter, T. J. Am. Chem. Soc. 2015, 137, 3775.

11. Bonet, A.; Odachowshi, M.; Leonori, D.; et al. Nat. Chem. 2014, 6, 584-589. 